\title{
altes lfaus!
}

fwiener Familiendrama in vier Gikten von Robert Salip.

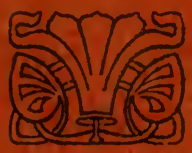

Dresden, Є. Pierson's Veriag. 


\section{the \\ university of connecticut libraries}

hbl, stx

PT2599S22E4

Ehrliches altes Haus! :

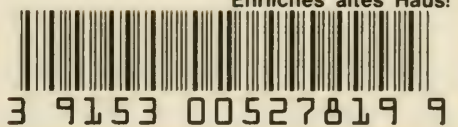

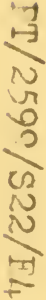




Ein ebrliches, altes நaus. 



\section{Ein ehrliches, \\ altes haus!}

Wiener familiendrama in vier Jleten

\section{hobent Salmt.}

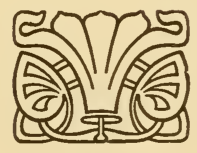

Dressen. - E. Pievion's Dewlas. 


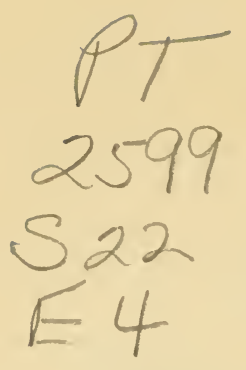

Arle Rewte vorbebalten.

Den Bühnen gegenüber als Manulf́ript gebrudt.

Das $\mathfrak{A}$ ufführungsredht für öffentliche und private Bühnen erteilt

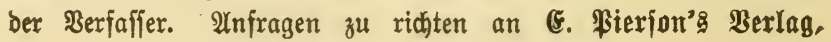
Dresben, Stephanieniträ̉e 69. 


\title{
Zerjonenverzeidfuis.
}

\author{
FerdinattD Fredinger, Fabrifant. \\ Marie, ieine Frau. \\ Pepi \\ Minimutud \\ Mitipi \\ Toni \\ Edubiaf, Deren Edhwiegeriohn. \\ Leffer, SBerfführer. \\ (5illi, Ibteilungsleiterin. \\ Dr. Şatidjef, Sildoofat. \\ Dr. İeger, 2lovofat. \\ Weterlit, Dienerin. \\ (Eit Sdjretber.
}

\section{3eit: (Segenwart.}

3wijchen bem zweiten und britten $\mathfrak{A}$ ft liegt ein Beit= raum von zimei Эahren, zwiichen bem britten und vierten Ilft ein folcher von jechs Jabren. 
2599

522

EY 


\section{Beideribung Der hnnoelinden Werionen,}

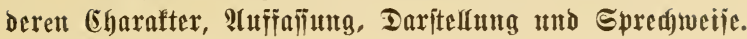

Ferdituand Fredinger, unterjeteter, breitjalulteriger Mann, Ende ber F̧ünfig, mit rotem, gejundem, Derbem Bejicht, lifitigen Diebs=

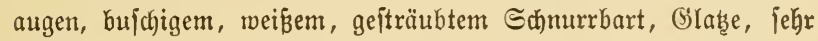
berneglich, gerieben, ipiţfindig, 5euchler, Berbrechernatur und um

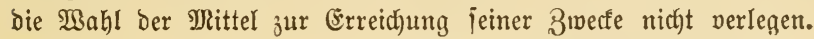
Mann aus bem unteren Bolfe, melcher emporgefommen nunmebr der Meinung ijt, mit feinem Gelde alles ereichen äu fönnen. Sit brutal, gemein utto fittlich verfommen. Spricht ben oroinären Biener Dialeft.

Marie Jrebinger, feine Battin, gediegene folibe Frau, grau= melierte Ђaare, franfę 2 (usjehen, lange bettlägerig, entwicfelt groje Siebe für iłre Sinder, ijt die perjonifizierte (Süte. Spridjt ben gemütlichen $\mathfrak{B i e n e r}$ Dialeft.

Pepi, deren älterer Sohn, junger fejcher Mann, Mitte ber 3wanzig, mit rotblondem Schnurrbart und Saar, leichtes Blut, echter Biener 5̧ausherrmjohn, guter fierl, nicht ichlecht, aber leichts finnig, unterbält jich gern, etwas apatfijd), aber leicht aujbraujend. Spricht den gentütlichen $\mathfrak{B i e n e r}$ Dialeft.

Maimutto, Deren jüngerer Sohn, junger hübider Maum, ca. 17 bis 18 Jalhre alt, bartlos, mit feinem (Sieficht, jchwarzem Şaar, Student, Joealift, guter, anitändiger Buriche, gebiegener Eharafter, hochintelligent, aber itolz, el)rgeizig und jähäornig, hegt groß̉e ßerebrung unD 2 (nhänglichfeeit für feinte Rutter. Spricht gutes Deutich mit weichen $\mathfrak{B}$ iener 2 Anflängen.

Mithi, Deren ältere To(hter, junge, fleinere, morserte Frau, ca. 30 Эahre alt, fchmarze Şare, gelber Ieint mit tiefliegenden, gierigen, falichen, jogenannten Eulenaugen, neibig, falid, gemein, Ginterlijtig, Seuchlerin; iteht zudem unter Dem (sinflup Sdubiats, ihres Mannes. Spridt befieres Deutich untermijat mit Wiener Dialeft.

Ioni, jüngere Tochter, gejchiedene, junge Frau, rotblono, Gübiches, feines (Beficht, jchlant und jän gewachien, beffere Natur, aber leicht= finnig uno lebensouritig. Nicht ganz verdorben. Spricht fo wie Mitįi.

Sđubiaf, Frebingers Sthiegerjolnn und Miţis Mann, ein= 
gewandert von ber Girenze ber Donaufürĩtentümer; langer, magerer Mifchling zwifhen utgarifhem Juben und Raizen, j(h)warzer, breiter Spib̧bart, fhwarzes, ftruppiges Şaar, gelbes, unreines Geficht, ab= geplattete Naje, Diebsaugen, ijt zu jeder Sannotat bereit, joferne

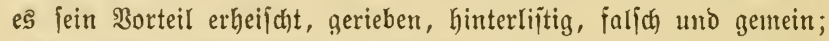
erbärmlicher, niederträchtiger Ȩarafter, zudringlich, wiro aber ठurch= ichaut und gemieden. Spridht ein leiernoes, jingendes Deutid), aber idnell und queticht bie Silben.

Seffer, Werfführer, gutgenährter, bicfbäucbiger Mann aนร ber Scefe bes ßolfes, itarker Bierziger, idharz, etwas meliert, mit jhwarzem, bujhigem Sd) nurrbart; aufgedunienes, gemeines, łlobiges, unbedeutendes (Beficht mit Bïundraje und fleinen, tiejliegenden

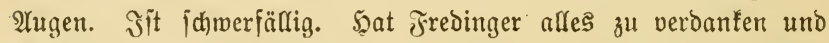
ijt um bes Bortei!s Millen zu allem zu haben. Gerviffenlos, jalecht,

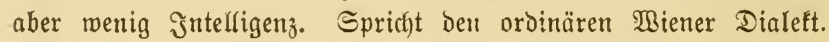

Cilli, 2lbteilungaleiterin und Fredingers Sonfubite, fleinere, unterjeţte, fababblige, faule, fhlampige Perion, Mitte ber Bierzig, blonogefärbt, aufgedunfenes, nidjtsfagendes Beficht mit boshaften, liçten 2 ugen, entfeţlich oumm, boshafi, eingebildet, moralija ver= fommen, jpielt fich aber auf bie anitünoige, beflere Jrau hinaus. Spricht den orbinären Miener Dialeft, den fie fith ju verbeffern bemülyt (nit falfher Betoming). SSlaubt ernitlich dus von ifr Seiprodjene.

Dr. Watidef, mittelgrojerer Mann, vollbärtig, unreines Blono,

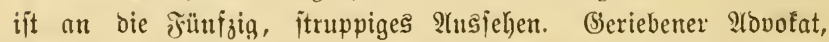
(क)lecht, heudylerifh, mit allen Salben gefchmiert, trägt womöglich Dunfle 2tugengläier. Spricht gutes Dentf(h, aber Iangian und ge= behnt, uno will hierourch Eintorud ausüben.

Dr. Jeger, gutgenährter, fđ̆malzig̨er, feinerer, betweglicher,

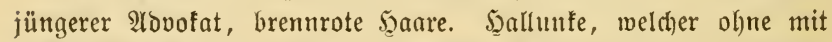
einer $\mathfrak{B i m p e r}$ zu zudfen die größ̄ten S(hurfenitreiche vol(fül)rt. Spridat gutes Dentid) und ift jehr zungenfertig.

Beterlit, Bedienerin, älteres, itarffnochiges $\mathfrak{W e i b}$, für belo zu allem $\mathfrak{z}^{\mathrm{h}}$ haben, ftiehlt, betrïgt, lügt, iit neugierig, idflecht, beuchlerifo und falich. Spricht orbintärent Wien.r Dialeft.

(Eill Ed)reiber, gleichviel wer, wontöglich harmlojer Menich. 


\section{(ivititev Alst.}

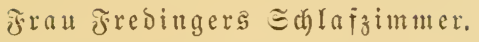

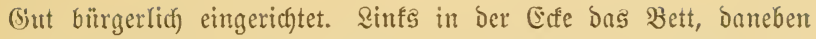

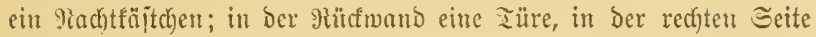

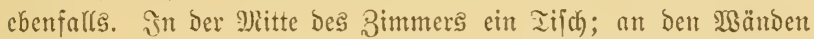

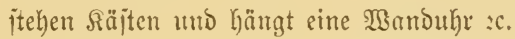

\section{Erite Szent.}

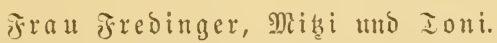

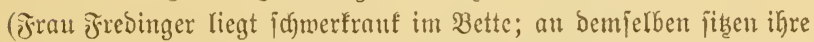
Töbter Mitsi und Toni und Gendyeln liebevolle Betreuung und

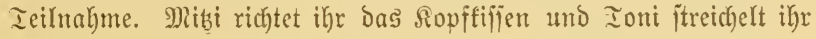
bie auf Der Bettoecfe liegende bando.)

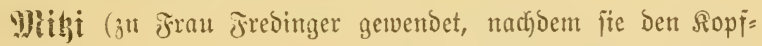
politer icheinbar geridftet Gat, in liebevolf tröjtenden Ione). Miutter, ijt bir jo leidhter? Miet wahr, jo? (ङ) ättet jorgiam bie Bettoeffe uno jtreichelt bie 5yano Der Frau jresinger.)

Frau Fredinger (ichmer atmeno). If bifiel, Mibi. Şch glaub' mir wirb fchon wieder befifer werden.

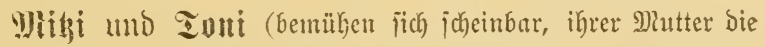
größ̈te S(ufmerfiamfeit зu wiomen, hordhen aber angeitrengt zur mittleren Türe (jin).

- Frau Fredinger (von neuer soffnung bejeelt). STber finder, viefleicht werb' ich bod) wieder gejund? Set wahr?

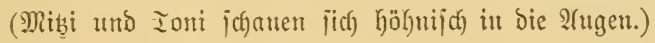

Salm, Ein ebrlides altes 5ูaus. 
Mitji. 2rber ja, Mituter, uno wirjt jehen, jehr bald jogar. (₹oni nifft zuitimmeno mit bem Siopfe.)

Frau Frreditger (ergeben uno jugleich) ermutigt). (Siott geb's!

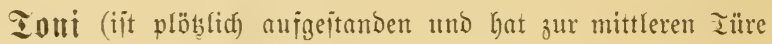
Ginausgeforcht).

Frau Fredinger (Gat bies bemerft und fragt). Mans tujt Du benn, Ioni?

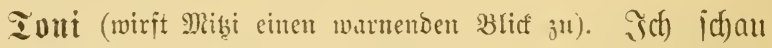
nur, ob der Maimuno idon fommt.

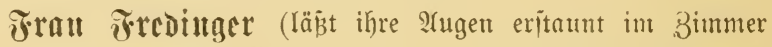
umberwandern). Ter Yaimund? Sa, mo iit er deun? Er mar ja grab' ba?

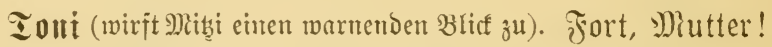

Frau Fredinger. Fort! Siso denn hin?

Ioni. Sn bie 2lpothefen iit er gegangen.

Trrau Jrediuger. In bie STpthafen? SBarum denn er jelber? Das Dienitmad, bie Heji, oder bie Feterlin hätt' ja auch gehen fönnen.

Toni. SBeiß̄t, Mutter, bamit bu's ichneller friegit ijt er jelber gegangen, er muß die Miedizin aus oer Stabt holen.

Trrau Fredinger (gerüfrt). (S)uter B̉ub! (Dam

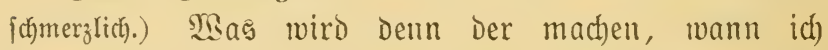
nimmermehr bin! Shoch jo jung, hat niemanden auf Der SrEelt als mie mich, und ber Sater mag ifnn net leiden.

Toni. NGer Miutter, wer wiro benn gleich an io mas Denfen.

Mhipi (eifrig ben Fnben aufgreifend). Iu braudbit bid) 
net zu fränfen, Miutter, megen dem Miaimund, wir merben ichon auf ifhn idfauen (immer eifriger werbent) uno mein Mann ijt ja auch ba, ber hat den Fiaimuno nod) viel lieber wie mir. SBir haben ifn alle gern, nur ber Sater mag ihn net, grad io wie ung. Wie möchten denn erit wir ausfchanen, wann bu wirflid) nimmermehr wärit. Der Bater mag uns icton gar net uno wir haben net viel.

Frau Frediunger (unterbrechend). Na, und das mas id) hab', bas Scaus?

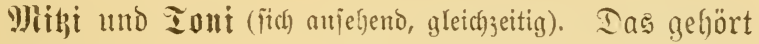
ja bir, Jutter!

Frau Frediuger. Stber ifr friegt's nach meiner.

Mibi. Mann ber Water nichts dagezen hat.

Frau Frediuger (erregt). Siam er ja gar nicht!

Mithi. D ja!

Frau Fredinger. Sisann id) es euch vererbe?

Mithi und Tout (unijono). Sa wann!

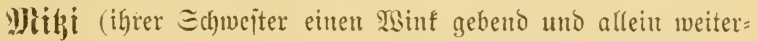

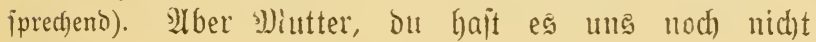
vererbt?

Frau Fredinger. Şd werbe es elld aber ver= idhreiben, idh habe eh' (d)on Daran gedacht.

Miiki (cifrig unt järtlich zu Frau ₹redinger). D Mlutter, ou bift io gut. (5)'idheio wär's ichon, wenn du es tätejt. Denn ichan, Mitter, wir find vier finder, haben ielber Sinder, uno wann bir, was (Sott verbüten ioll, mirflick) einmal was g'idhel)'n möbt' und bu hättejt fein Iejtament gemacht, jo nehmet's ber $\mathfrak{B} a t e r$ und wir hätten gar nidhts, nur bamit er alles feinen Reuten, ber Cilli, der 
Sathi und ben andern geben fann. Du fennit ifn ohnedies!

Frrau Frediuger (mit der 5̧atb abuinfent, bitter). Sei rubig, Mibi, und red' mir non biejen Reuten nidjt, bie mir mein ganzes Reben vergiftet haben. Эch wein, was fie mir angetan haben uns idh weip auch, was mir elter Sater angetan hat. $\Im$ sh) lenn' ifn nur zu gut. Mtber er wiro euch nichts itreitig machen fönnen.

Milji (troitlos). Er wirb's aber boch tun!

Fran Frediuger (bejtimmt). Tein, meil idf) mein Ieitament machen merbe!

Mhiti. Du mollteit, Mutter!

Fraแ Fredinger. Ja!

Mithi (eifrig). Da fönnten twir ja ben Dr. Seger holen laffen.

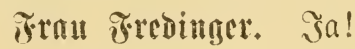

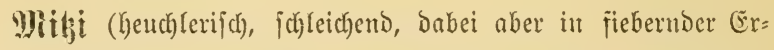
regung). Mutter, ba fönnt man ifn ja gleiç holen laffen.

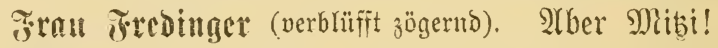

Miipi (jie unterbrecheno). Эch mein' halt, weil wir grao' banon reden, Miutter. MEenn's bir net unangenebm iit.

Frat Frediutger (ichwantend). Ta, bas grad' nicht.

Mlitji (ipringt auf). Ia mero' id) ifn lieber gleich holen. (eäuft zur mittferen হäre; $\mathfrak{a b}$.)

\section{3rueite Szcne.}

Frau Feroinger, Ioni.

Trtan Tredinģer (peinlich eritaunt). SBarum lauft Dern die Mibi gar io idhell bavon? Ees hat ia noch Beit! Mieinit nicht auch, Toni? 
Toui (verlegen, idjeinheilig). D ja, Miutter! Beit bätt's genug. Ess wäre ja überhaupt nicht notwendig gemejen, benn bu wirit ja wieber gejuno werben, Miuter. Es ijt bir ja heute idjon viel belfer.

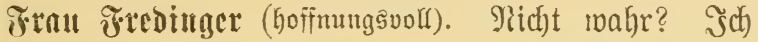

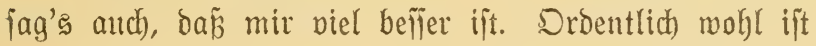
nir.

Toui (beteuerno). Das fieht man, Mutter, und es ijt aud feine (Sefahr ntelyr, aber bie Mibi glaubt halt, wenn bu faton einmal babei bijt und ein Ieftament machen willit, daßj man's gleich abmacht, bamit bu bich nicht mieder mit joldhe unangenebme Sachen abgeben muñt. Die Miki meint bir's ja gut, Miutter!

\section{Frall Fredinger. Bstaubit, Toni?}

Toni (Lebhajt betenerno). Iber Mntter! Sie hätte ja Keinen andern (S)runto.

Frau Fredinger (beuthigt). Sha, bann foll's balt

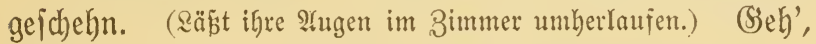
Ioni, joua' nach), ob alles zujammengeräumt ift uno räum' ein biffel nach, bamit es ordentlich ausichaut.

Toni (iteght auf und räunt auf). (Sern, Mutter, werd's gleid) machen.

Frau Fredinger. Denn id) Denf', ber Doftor wird bals fommen.

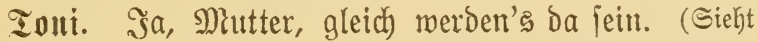
nad) ber uhr, fitch vergefiend.) Sie jollten eh' fafon ba fein!

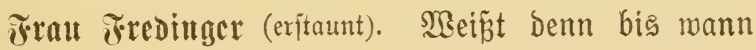
fie fommen?

Tont (jich bei ihrer Unüberlegtheit ertappend, heudflerif (d)). 2(ber nein, Miutter, ich babe nur g'meint, fie föunten 
ichon da jein (räumt weiter auí), benn es ijt ja net weit zum Dr. J̌eger, der woohnt ganz in der siähe. (3ur Seite.) Ia hätte id) mich aber bald ordentlich verichnappt. Mo jie nur bleiben? Mir haben boch alles mit Schubiaf abgemacht. (३um ßette Der హrau Frebinger gehend,

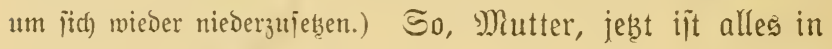
Dronung, jeb̧t fönnens ichon... (Ẻs flingelt.) 2lth, mir

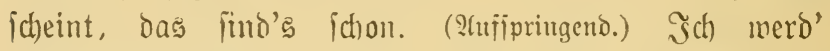
gleid) nachichauten, Jutter, mer es ift. ((ङegt zur züre und will jie öff̈nen. Ĵn biejent Montent wirb biejelbe von auß̄en geäffnet uno herein tritt Mitbi, hinter ifr Dr. J̄eger uno ihr Meann.)

\section{Iritte Szene.}

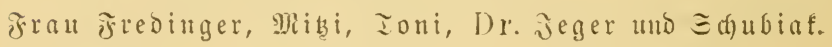

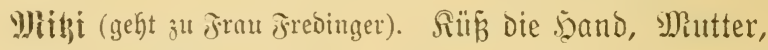
da bin ich ichon micber. Bufällig hab' ich meinen Mann 'troffen und ba haben wir bell Dr. Seger gleich miteinander geholt und gleich mitbracht.

Frau Fredinger (widerwillig). Deinen Mann?

Mithi (ausıeidyend). Niein, den Dr. Säger, mein Mann ift nur mitgegangen.

Frat isredinger. S(ber beinen Mann brauchen !vir ja net.

Miłji. Mber Mutter, muñt net bös jein, dañ er mitgangen ijt. Ese ijt ja nur wegen bem Ioftor.

Edulbiaf (aun bette treteno). (S)uten Iag, Edbivieger= mama, wie iteht bas werte Befinden?

Fran Trredunger (nifft mit dem Ropfe). Danfe, gut. -

Sdjubinf. క̧h habe meine Frau mit Dem Doftor begegnet uno ba habe ich mir erlaubt mitzugehen. 
Frau Frediuger (mübjam ifren sopi hebeno). SBo ijt Der Serr Doftor?

Mihi, (zeigt auf Dr. J̃eger). Djier, Mintter!

Dr. J̀fger (mar in achtungşvolfer Entfermung vor bem

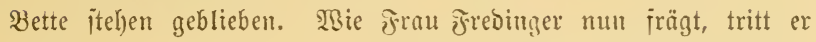

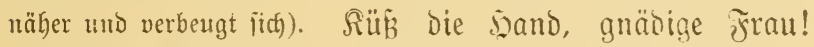
Frau Frroinger (nifft). (S)uten Tay, 5err Ioftor.

Dr. J̀ger. (S)näbige Frau haben mich) rufen laîten? Frau Fredinger. Şa!

Dr. Jiger. SRie mir şbre Trau Iochter mo Der Sert Echniegeriohn fagten, münichen guäbige Frau teitamentariiche SAnorbunngen zu treffen.

Tran Fredinger. כ̌a, Serr Ioftor.

Dr. Jeger (öfinet jeine Sajche unto entrinumt derjelben ver=

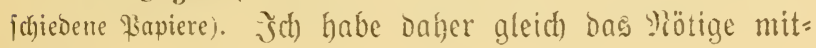
gebracht.

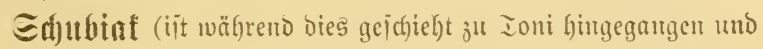
frägt fluijterno). Iie \&uft ijt boch rein?

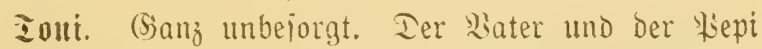
fund im (Siejdäit uno bie Iienjtmadn hab i meggeidjicft.

Edjubiaf. Unt der Tiaimunt?

Toni (bögniich). Der ift in bie Etabt, um bie Miedizin für die Miutter. (5̆b̆nifo lächelno.) Die Mitbi hat's ja ertra atsg'id)itt.

Edjubiaf (Gändereibeno). Da find wir alio unoẹtört?

Tont. Mgann nidjts dazivijchen fommt, ja. (Schnefl.) Şörît, Echubiaf, haît ou mit Dr. Säger alles abgemad)t?

Edutuinf. Ifles! Ias Ieftament wiro fo gemadyt, 
wie wir es braudben. Menn bie Schiviegermama niddt zu viel Mideritand leiftet!

Mithi (ruft). (Seh', Toni, gib bie Tinte her und eine Unterlage, bamit ber seerr Doftor ordentlid) idhreiben fann.

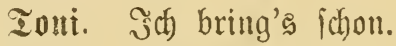

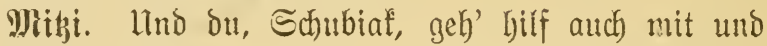
rüct't's ben Tijch her.

Tout (bringt Tinte und UnterIage und Schubiaf ergreift mit

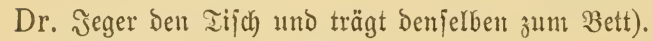

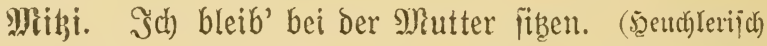

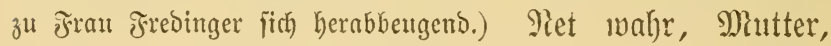
fo ijt's bir red)t!

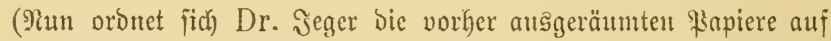
Dem Iifche, richtet fich alles zum Scfreiben zureecht und indeffen jeţen Ioni und Schubiaf igr (Sepprächl Leife fort.)

Tout (zu હchubiaf). Sૂann aber bie Mintier net will?

Gdjubinf. S⿰氵 vertauf cht.

Tout (erjaroden). Wertaufdht! Das Ieptament!?

Edyubial (idnell). Der Dr. Seger hat's fabon fix und fertig ba. B̧ringen wir bie Schwiegermama nicht bazu, bas Iejtament fo zu machen wie wir wollen, fo taujcht er's einfach um und lap̃t ihr bas faliche unter= ichreiben.

Tont. Stber wenn's d' Mutter mert'?

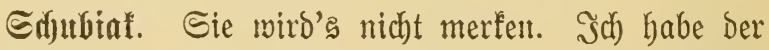
Mitici unten fdyon alles gejagt. Sie hat fich barum fo hingefebet, baj fie's verberfen fann, wann'e ber Doftor umtauficht. 
101. Jeger (Gat feine \$orbereitungen beenbet und wendet fid an Frau Freoinger). $\mathfrak{A l j o}$ wenn gnäbige fran ge= ftatten, fönnen wir jekst beginmen.

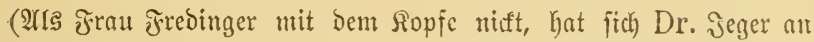

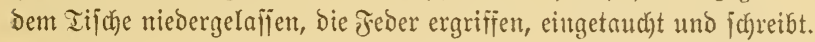

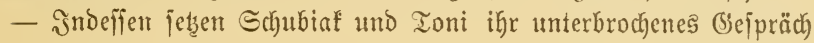
fort.)

Toni (зu Schubiaf). Maa fteht bem in bem falichen Iejtament?

Edutbiat. SMie wir's beiprocken haben. Ias Szaus zu gleichen Teilen, es Darf aber bir und meiner Fraut nichts abgezogen werden von den vierzigtaujend (S)ulden, die wir ertra befommen haben.

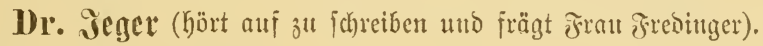
(Snäbige Fraแ heiß̄en?

Mithi (ifherf). Marie Frebinger.

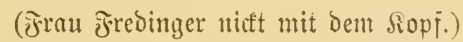

Dr. Jeger. Sitte, guäbige Frau. (ভçreibt.)

(Schubiaf mo Ioni iprecten weiter.)

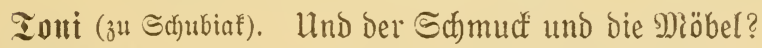

Gdubinf (zu Toni). Ssebört alles nur bir und meiner Frau! Dann befommen alle fitinder taujend Bulloen bar auf bie Şani, mur ber Maimuto nidjt. -

Ioni. Db's aber gef'n wirb?

Sdubiaf. Marum nicht? MSenn nichte bazwijchen fommt. Stber vielleidft ijt's gar nicht notruendig und bie Sd)wiegermutter meigert fidh nicht, benn wie mir meine Frau gejagt hat, joll fie (d)on jebr idjwach jein. (Wie Dr. Seger muntmehr zum iprechen anfebt, horchen Toni und Schubiaf intereffiert auf und wenden fich jum Bette ber Frau stresinger.) 
Dr. Jeger (hat jein Sdjreiben unterbrochen uno jagt ju

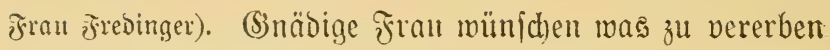
und wen zu Erben einzulezen?

Trrau Fredinger (will ipreçen, aber Miţi fommt igr z̧uvor).

Mithi. Die Mitter will ung Den findern ihr şaus. verichreiben.

I)1. Jeger (vor Mitisi fich leicht verbeugeno unto itid) an israu Jrebinger wendeno). Stimmt bies, gnäbige Frau?

Fron Fredilnger (mit dem siopie niffend). Şa, jerr Doftor.

Dr. Jeger. Siselches D̃ans, guäbige Frau?

Frant Fredinger. Miein Şatts in der \&anogaffe.

Mithi (iie unterbrechend). No. 107, das im (Sirund= buch) auf bie Mutter g'idrieben iit.

Dr. Ieger. Ianfe iefr, guäbige Frau! ('Birft einen $\mathfrak{B}$ licf auf Schubiaf und idgreibt, leije vor fich himprecthendo.) Bermad)e id) zu gleichen Ieilen - (Sid) an šrau srebinger wendento.) (Sinäbige đrau erlauben, allen Shren Rit!dern zu gleichen Teilen?

Frau Fredinger. รa!

Dr. İger. Bitte, gnäbige Frau, wie heiß̧en Эhre Sinber, ba $i d$ b biejelben namentlid) anführen muई́.

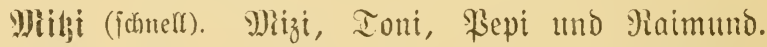
Sdhreibens nur, seer Doftor, allen meinen findern zu gleichen Teilen ohne গIbjüge. -

Frant Fredinger (Зuhöreno mo abwinfeno). 5ुerr Doftor, bas gebt nid d)t!

Dr. Jeger. Şas, gnäbige Jrau?

Mliki (verlegen). Mase meinit benn, Mutter?

Frrat Trediuger (ॐu Mitzi). Du bait was g'jagt 
ofne $\mathfrak{A b z u ̈ g e ? ~ D a s ~ g e h t ~ n e t , ~ D e n n ~ b a n n ~ f o r n m e n ~ m e i n e ~}$ Buben zu furj.

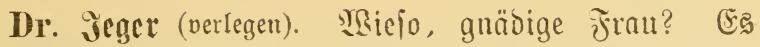
iit boch ielbitwerîtändich, dañ Sie Şhren Rindern das ড̇aus vererben wollen, ohne ihnen Irbzüge ju machen.

Trrat Jredinger (entergiifg). Sa, alle iollen basfelbe friegen, aber bas, was meine Iöchter fidon hernuken haben, mur abgezogen werben.

Mithi uno Toui (weinerlich). Stber Mintter, wir haben eh' nichte.

Dr. Jeger (einoringlich). Ntber guäbige frrau merben Dod) Shren Töbtern nid)t bie erhaltene Mitgift in $\mathfrak{l}(b=$ zulg bringen.

Frau Fredinger (bejtimmt abinelyend). Won der Mit= gift red' ich ni(f)t! Itber bas, was fic aup̃erbem in den lebten Sabren von mir allein befommen haben. Sh) habe desmegen auf nein nans t0000 (Sulden aufgenommen und bas haben unr meine jwei Söchter 'friegt.

Toui (weineno). SAber Mintter, jebt willit uns bas abzieben.

Mithi (ebenfalts weinent). Tein, dañ bie Miutter io jein fann. Mas jollen wir Dent anfangen, wenn uns Das abgezogell wiro.

Toni. Ia friegen bie Bub'n viel mehr wie mir und bie tun bas (Sseld höd)jens verwichjen wie der \$epi.

Frau Fredinger (wiberitrebend). SBarum follen benn meine 3 uben weniger befommen, weil ber Kepi ein wenig leicht ijt? Hno gar ber Haimumb? Der ijt 
Doch fein leichtfinniger Mienich. Der barf nicht zu furz fomment.

Mitji. Slber Mintter, g'rad ber braucht's gar nicht. Der ßater muß́ für ilyn forgen, bis er großjiäfrig ijt, Da hat er ja an ben Binfen, bie er fich eripart, ichon fo viel, wie wir Frattenzimmer, wir fint elj' fo arme (Seje)öpf' und haben felber noch finder.

Toni. Die Mitizi hat wenigitens noch einen Mann, ich bin aber gefdebeden und iteh' mit mein Simb allein da uno hab baun gar nichts, benn von meiner Mitgift und bem, was du mir abzieben willit, ijt nichts mehr

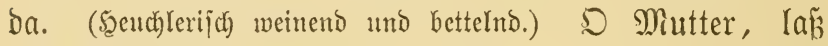
uns bas und ziel) uns nichts $a b$ ?

Mithi (ebenfarle bittere Iränen heuthelno). D Mutter, la

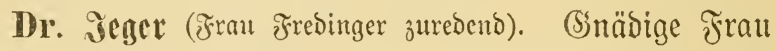

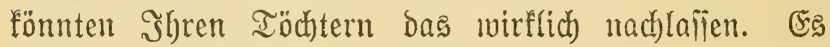

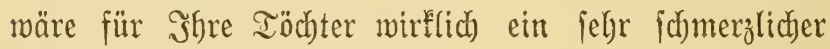
Entgang und כ̧̧ren Eöhren binfte es aud nidjt io weh tun, wie \$fren Iöbtern, notabene, ba ber eine Shrer Söhne etwas leicht jein foll. -

Fran Fredinger. 2 ther Scerr Doftor, wegen bem Faimuno! ich bring's nid)t über mein (Siewififen!

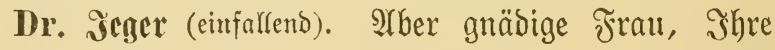
Töd)ter werben ichon bafïr jorgen, daß er nicht zu furz $\mathfrak{f o m m t . ~ - ~}$

Toni und Mipi (beteucrno). Und wie wir auf ifn idbauten werden, Mutter! Stber wann lins bas abge= zogen wirb?

Dr. Jeger. (Ssnäbige Frau, es ijt auch am ange= 
zeigteiten, wenn Sie es jo ließ̧en, denn wenn Sie nidjt

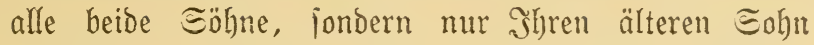
ansnebmen, fo wirb biejer um io ficherer bagegen proteftieren und bas Iejtament anfechten. $U$ m diez zu berbindern, wäre es wirllich am vorteillafteiten, wenn bie Falifung biejer Stelle fo bliebe. -

Mithi (einoringlidy). ङ(hau, Mutter, Der 5̧err IoEtor hat yanz Piecht. Es iit wirflich am allerbejten, wenn es jo bleibt und wir werben auf ben Raimuno ichon idfauten. (Sidf an ifren Mam mentend.) Midft twahr,

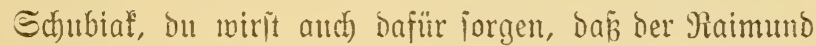
nicht zu furz lommt.

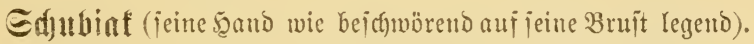
Das mirb itets meine gröpte Eorge jein, Echivieger= mama. Sie brauchen fid) nicht zu ängitigen und wenn ich es bem Siaimuno von meinem eigenen Bermögen eriesen mü̈̆te, denn .....

Witbi (abminfend und einfallend). Ias mird ja gar nich)t notwendig jein!

Edfubinf (ihr zuwinfend). Db notwentig ober nidht, Haimuno muj jein ganzes Erbe befommen und er wiro eS befommen, Edhriegermama, benn id itebe bafür mit meinem Eyrenmorte ein. Sino Eie bamit jufrieben, Schiviegermama?

Fran Fresintger. (E) ift fidon gut. J̌d) glaube es Sthnen ja, wenn Eie es mir hier veriprochen haben. Dr. J̌eger (eifrig). Stlio gnäbige frau münichen,

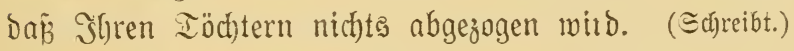

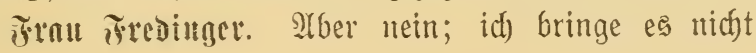


über mein (Servifien, ich faun boch meine Buben nicht ichäbigen, ১as muß̉ ben Mä̈beln abgežogen merden.

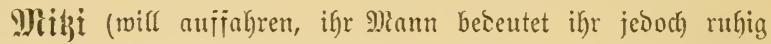
zu fein, jie verifteht und ichweigt).

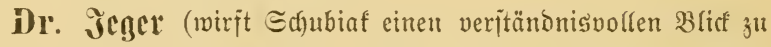
uno jagt zu ร̧rau శrebinger). Run, menn gnäbige Frau burchaus nidft anders münidjen, (ipridst idgreibeno) alio abzüglid) Der 40000 (S)ulden.

Frau Frediuger (nidft bejaheno).

Dr. Jeger. Şabell gnäbige Frau fonit noch etwas bem Ieftament beizufügen?

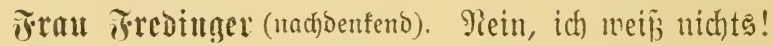

Mikj. N(ber Der ভchmuck, Mutter?

Frau Fredinger Ier g'hört ohnedies ench).

Ioni. Uno bie Mröbel?

ซrau Fredinger. SAluch!

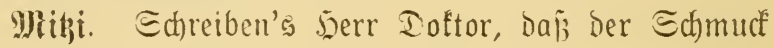
uns g'bört. (Deutet auf Zoni unb jitr) ielbit.)

Frau irredinger. Euch allen, auch meinen Buben.

Mlibi (will auffabren, ihr Mann winft ihr wieder. Sie veriteft und idfweigt).

Dr. J̌eger (veriteght ebenfalfs und jagt). Mllio bie Mö̈bel

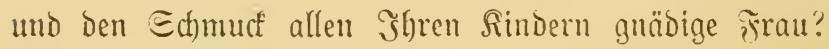
Trau Fredinger. Sa!

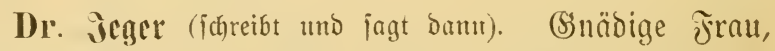

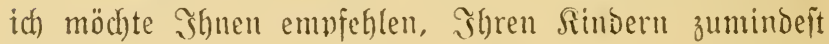
Den älteren, eine fleinere Eumme zu tejtieren, welche gleich) bar auszuzahlen wäre, bamit bieielben über Bar= geld verfügen und nicht auf ben serrn (sicmahl ans gemiejen fint. 
Ioni (eimfalleno, eifrig). Sa, Mintter, denn Der Bater gibt uns nichts.

Dr. Jeger. SEenn gnäbige frau niduts dagegen haben, iagen mir 1000 Suldon.

Frau Frroinger. (S)ut, berr Doftor.

Mr. Iegrer (ịchreibt dies nicber).

(Эn biejen Moment pifintet fich leife bie gegenüber liegende Seitentüre und Peterlin itectit vorifichtig ben Sopf herein uno will herein.

Wie fie bieje (sruppe ficht, jtubt fie.)

Feterlit. $\mathfrak{i t h}$ da bin i grad no z'rechtfommen. Die mad)en ja 's Iejtament. Drum hab'ns jelbit mi

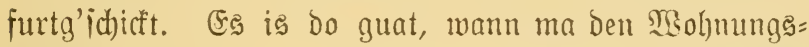
ichliitiel mit hat, io haben's mi net fummen g'bört. Das muá̉ $i$ glei ben gnä Seern jag'n, bas wirb mir wieber was eintragen! (Sdglię̧t vorfichtig bie Türe, ohne beme1ft worden ju jein.)

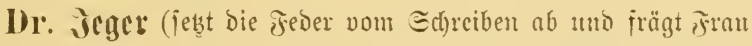

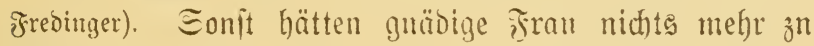
ermähnen?

Mithi (idnell). Tiein, jeerr Doftor, nichtó mebr.

Frau iredinger (rubig mit dem Sopje verneineno). SRein!

Dr. Ieger (ichreibt nod) etwas, wirft hierauf Miți einen veritänonişvollen 3licf $3 u)$. $\Xi_{0}$ jeķt mären wir fertig.

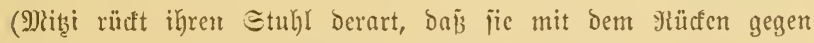

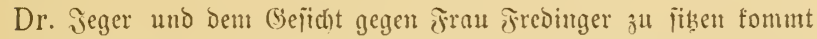
und beginnt fich lebhajt mit derjelben an bejchäftigen, um beren Suimerfjamfeit von bes Doftors Iun abjulenfent.)

Dr. Jeger (weldyer auf bas Geichricbene ein söffjpapier legt und bas faliche Ieịtament zum 5ervorziefen vorbereitet). M̈sunichen gnäoige Fran es jelbit noch einmal burch)= julejen, oder joll idh es vorlejen? 
(ふ̧rau Jrebinger nickt.)

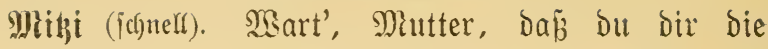
Müh' eriparit, id) werde es burcblejen. Piet, Mutter?

\section{Frnt Frebinger (ahmungẹtos). Meinetrogen.}

Dr. Jeger (zieht unter Dem \&öjichblatte bas vorbereitete faliche Ieitament herrus uno legt es vor Mitizi hin). Bitte!

(2Bähreno M) Mtiţi başelbe lieit, nimmt Dr. Jeger bas unter bem

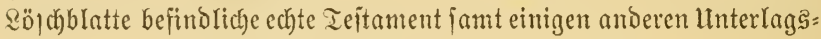
papieren uno räunt es in jeine Iafdje.)

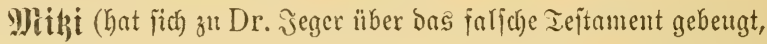

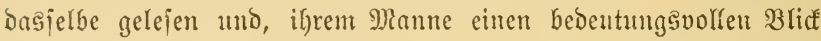

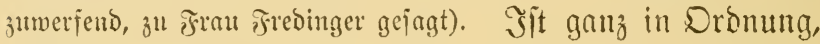
Mitter!

Dr. Jeger (taucht hierauf die Feder ein, nimmt bas faliche

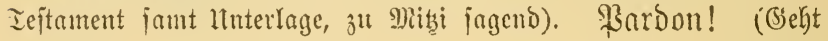

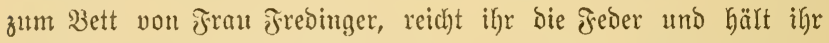

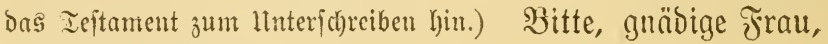
zu unterichreiben!

Frrall Frediuger (nimmt bie ǰeber, unterichreibt milbjam).

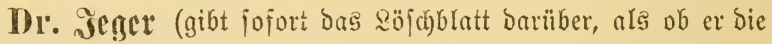

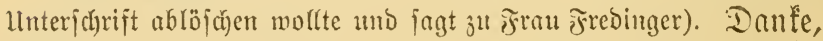

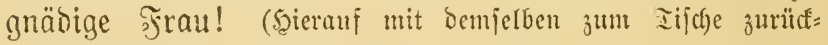
fomment, faltet er es zujammen, entnimmt feiner Tajdje eine Enveloppe, in werdhe er bas Teptament gibt, nimmt Siegel unto Siegelitod auts feiner Iajaje, verifegelt es und jagt zut grtau

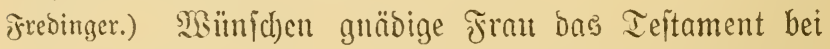
iich) jtl behalten, ober foll idh es bei (siericht finterlegen, ober es jelbit in 2 tufbewahrung nehmen?

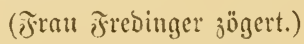

Mitiji (einfallento). Beim (Serid)t hinterlegen, Mutter! ?ididst im saus behalten, es ift wegen bem $3 a t e r$. 
Fran Fredinger. Es if mahr. Niehmen Sie es mit, berr Doftor. Ese iit am beiten io. -

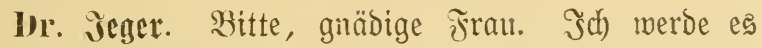
bei (S)erid)t hinterlegen. (Dr. Jeger partt jeine ভad)ent zu= jammen.)

Jrau Fredinger (ruft reiie). 5̧err Doftor!

(Diejer bört nidgts, aber Miţi hat es gehört.)

Mitpi. SBillit bu noch was vom 5errn Doftor?

Frant Trredintger. $\mathfrak{s}$ !

Mlibi (३u Dr. Jeger). 5eerr Ioftor, die Mitter möcht noch was pon §gnen!

Dr. Jeger (nahezu angezogen). Bitte, gnäbige Jrau wünichen?

ひrtau Fredinger (mit tränenumflorter Stimme). Şerr Doftor, nicht wahr, Sie ichauen, bá̉ mein Haimund nich)t zu furz fommt?

Dr. Jeger (verlegent, faß̈t firch aber bald und jagt betenternd).

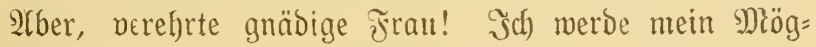
lichjtes tun, bamit bies verhindert miro. Şhre Iödhter, jomie ber Serr Schwiegerjohn merben aud ifren ganzen Einfluz in biejer giidhtung geltend madhen.

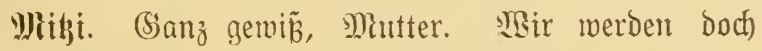
nicht mollen, Daßj ber jüngite, ber jo ein orbentlicher Burich ift, net fein Sachen friegt. -

Toni. Du fannit ruhig iein, Mitter. Misir werden tun, was wir mur tun fönnen.

Edjubiat. Shuniegermama, es wird mir bie Sorge um Haimund Shr teuerites Bermächtnis fein. Frau Fredinger (weint). Seer Doftor! Er ift fo jung noch!

Salm, Ein chrlides altę saaus. 
1)r. Jèger (berufigend). H(ber, gnäbige fraıt fönnen getroit icin. Eie haben bie Nerficherung Şhrer Iöd)ter und Эhres Edhriegeriohnes. Sie haben mein Mart, gnäbige Frau fömmen fid) ̈̈berzengt halten, Da $\tilde{\mathcal{B}}$ alles nur z̆u giaimunos Bejten geidjehen miro.

テัra Jrenitger (weinend). Mein armer Bub!

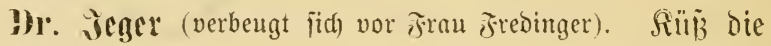
Şand, gnäbige Frau!

Fran Fredinger (ihm zumintend). Seben Sie wohl, Sherr Doftor!

Dr. Jeger (verbeugt fich vor Mitai uno Ioni). Silïb bie Ђand, meine Damen!

Mithi und Tont. (Suten Iag, jerr Ioftor?

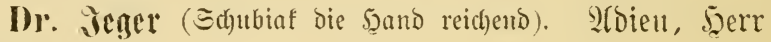
Schubiaf! !

Edubinf. Sroieu, 5err Ioftor!

(Dr. Jeger gebt, von હ(d)ubiaf begleitet, zur 氵itre.)

\section{Sierte Sine.}

Die sorigen und weterlin.

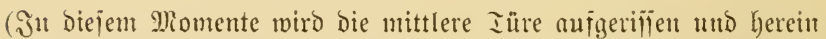
tritt \$eterlin, marfiert überraichung, als fie ben Doftor unb alle fielgt. Sie winft Mgiţi heran, weldhe fofort hinläuft.)

Mithi. Mas iit Denn?

Heterlin. Sunge jrau, oer alte jeer fommt herauf! Mitji (verblüfit). MSas? Der Water.

Peterliu. sa, glei muaj er da iein, er is grad um bie Ecfen bogen, wie i raufgangen bin. sismu $i$ g'wust hätt, junge Frau, Daj was abj'madjen haben, bätt $i$ illn vielleid)t auflyalten fömnen. 


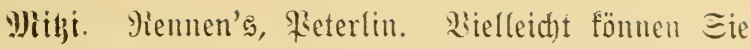
ifn noch a bififel aufhalten, bis menigitens ber Ioftor fort iit.

Wieterlin (macht, als ob iic es recht eilig madhen würoc). Sa, junge jrau, gleich), vielleich)t geht's no! (Memnt zur Türe Ginats.)

\section{(Man hört jđd)å̃ läuten.)}

Mihi. Er ijt ichon ba, ber Sater.

Edubinf (eritaunt). Ier Bater? Mgiejo weiß̄t Du es?

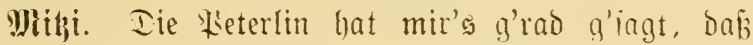
ihn unten nod) erblicft hat.

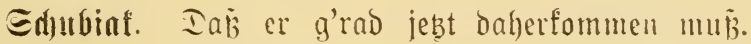

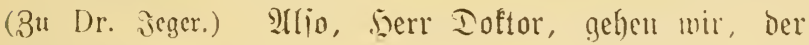
Schmiegerpapa iit's.

Dr. Jeger (verbeugt itd) nodfunals vor jran istebinger,

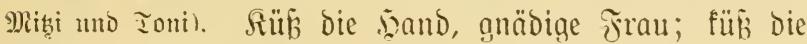
5zand, meine Damen. Sch empifehle mich, meine Iamen.

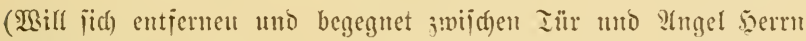
Frebinger.)

\section{Filnte Ejenc.}

Die sorigen und jrebinger.

Hetcrlin (blift f(d)adenfroh burch) bie Türe).

Ed)ubint. (S)uten Tag, Edhwiegerpapa.

Fredinger (cintretend). (Suten Iag, Schubiaf. 将as madhen Eic bier? (Den Dr. Jeger eritaunt aniehend.) Llnd Der Serr? Ein neucr Doftor?

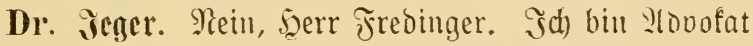
uno habe Die lebstmilligen Rerfügungen Şhrer F̂rau (Semahliu aufgenommen. 
Fredinger (bijitg, mištrauija). So! So! $\left._{0}\right)$ Uljo Teitament iit g'macht worben. (3u jeinen beiben Iöbtern

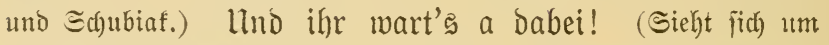

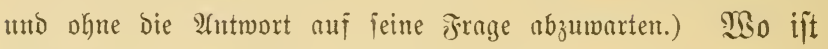
Mainund?

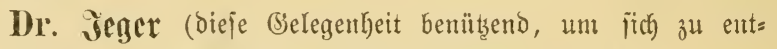
fernen). Empfehle mich, 5ूerr Frebinger! (2(b.)

\section{Eechjte Sjene.}

Die 3 origen ohne Dr. Jeger.

Fredoinger (nachrufend). Iroieu, 5̧err Doftor! (Sich

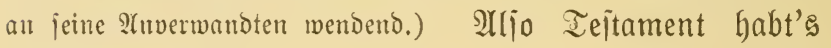
g'macht? io, io.

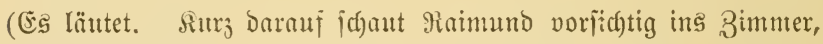
bie Mebizinflafche in ber 5̧and und will Miţi juminten. Wie er

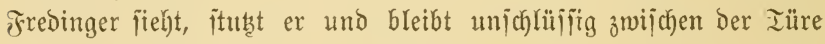
itehen.)

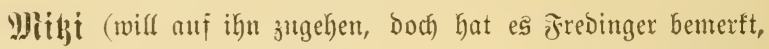

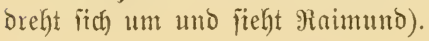

\section{Siebente Szente.}

Die 3origen und Ma

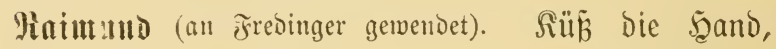
Water!

Fredinger (boshaft). গIlh! Du bijt a ba? Şajt a mitg'bolfen.

Minilutud (eritaunt und entrüitet). Mitg'bolfen bei mas? Sid) bin gerabe gefommen.

Fredillger. Эa, wo warit denn?

Minillulti. Ein wenig fort. 
Frreduger (gedefint). Uno bu bait die Miutter allein laijen?

Minimund. 2 ber nein! nein. (unjolüifig.) Itber wenn idh es idfon jagen muñ, die Medizin für bie Mutter hab ich aus der Stabt geholt. -

Fredinger (ïegt ihn an). Die Medizin! NEelche Medizin?

Matmund (ihm bie Flajaje zeigeno). Dieje hier.

Jredeinger (nimmt ifm bie Medizin aus der Şand, ichaut jie an.) 2rljo bie Miebizin hajt geholt? Arber bie ijt eh' erit gemacht worb'n.

Manimund (verblüfït). Das ijt nicht möglid).

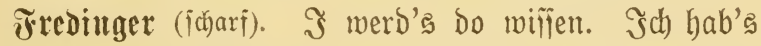
ja jelbit durch ben Szausbiener geitern holen laifen. Şat bie Miutter woll'n, ba ́a bu's holit?

Minimund (abwebrend). Atber nein! Die Echweitern haben mich gebeten.

Frediuger (z̆ Miţi und হoni). Marum ijt benn fa Dienjtmadl gang'n.

Mithi. Es mar feine ba.

Frediuger. So! fo! Mnd da hat's g'rad fo prefifert, wia net amal ane von ben zrwa Mabln ba war. Da hätt's ja 孔u mir munteridjicfen fönnen. Und bann

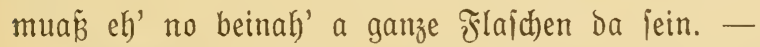

Minimund (einfaffeno). Siein, Sater, es war nich)ts mefr ba!

Fredinger. Mুajo net!

Raimund. Skeil mir die Mişi felbit bie leere Flaiche mitgegeben hat.

Fredinger. Die Misit? 


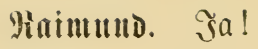

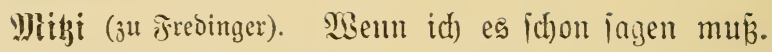
Das Flaichl iit umgefamen und bie Medizin ifit gleich ausg'ichütt g'mef'n.

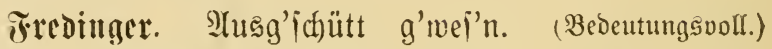
Sagen wir liaber ausg'ichütt woro'n. (3ur Seite.) So

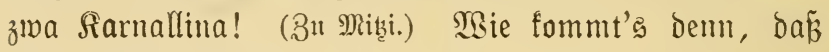
Dein Mann ba iijt?

Mithi. Zufüllig ift er herfommen.

Jredinger. Zufüllig! (Bur Seite.) Der Rampldtab!

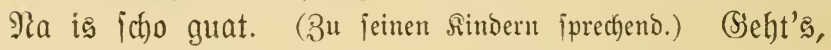
finder, ich hab mit eurer Mitter was j'reben.

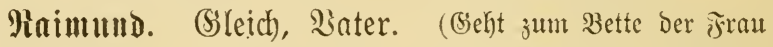

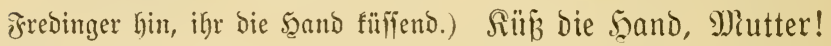
Mie geht's Dir Denn?

Frau Fredinger (riebenoll). SBart du Fiaubersbub! MBo wwarit du benn? Sich io Davonzuldhleidfen uno jeine franfe Miutter allein laîjen? Das hätt' ich mir von meinem Maimuno nicht gebacht.

Manimuแd (Iächelno). 2(ber Miutter, ich) wnar ja mur ein wenig fort.

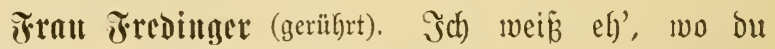
marit. Die Miedizin haît g'holt für dei franfe Mutter.

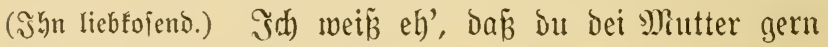
hajt und fïr fie alles tuit.

Minimund (lä̈heIno meich). Yieg' bich) nicht auf, SJutter.

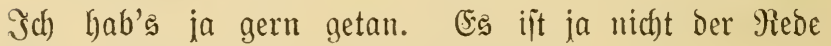
wert. MSann's bir nur bilft, Miutter?

Fredoinger (welcher bis bahin teilnams̆los zugehört hat, un=

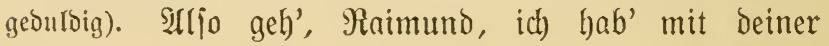




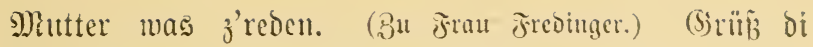
(Siott, Miariedl. Tia wie geht's dir?

Frau Frediuger (Ieije iprectend, in erregtem 3uitand zu rainumb). B̧leib' ba, Yiaimumo, uno la ja mich nicht allein mit'm Bater; er iefiert mich.

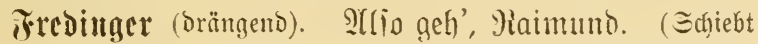

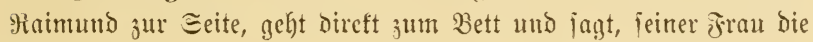
5̧and reichent.) Arlitern, Mariedl, wie geht's da denn.

Fran Frediuger (ängitlich uno jurïfflyalteno). Schlecht, Fredinger, fibled)t!

Fredinger. $\Xi_{0}$ ! (Sieft cinent Moment uachbenflich) vor fich bin und jagt ju jeinen Sindern gewendet.) Rinder, geht's 'naus, ich bab mit eurer Mintter was j'reden.

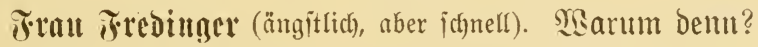

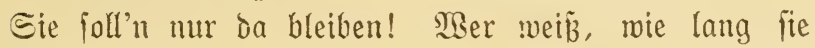
mich) nod) haben! Şas willit benn, Frebinger?

irredinger. 3'reden hab' i was mit bir utto do

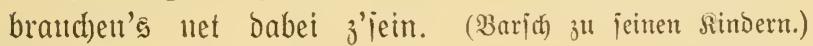
Alio geht's 'naus, finder.

(Dieje jehen ihre Mintter an und gehen zögerno eines nadh bem

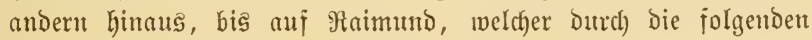

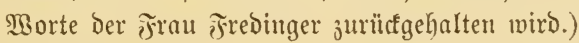

Frau rredinger. Der Faimumb foll da bleib'n, wann idh was brand).

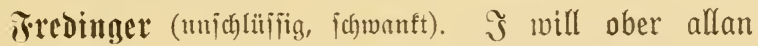
mit bir reden.

Frau Fredinger. Er fann fich ja zum Fenjter itell'n, do hört er eh' nichts.

irroinger. Na, meinetwegen. Do geniert er mi baun net weiter. (3u Raimun gewendet.) Du fannị do 


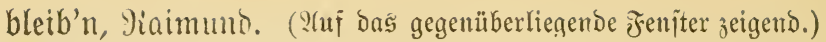
Sely Dort zum Fenfter hin, Denn i hob mit ba Muatta was z'reben, wann ma di braude'n, wer'n ma bi icho ruaf'n.

Minimund (wirft einen vieljagenden Blicf auj feine Mutter, welche nicft, uno geht mit ben an Fresinger geridbteten $\mathfrak{B}_{\text {orten) }}$ Bitte, Bater! (zu Dem gegenüberliegenden Feniter, wo er fïh) Ginter ben \$orbang itellt uno Ginausblicft).

\section{Aldyte Szene.}

Frebinger, Frau Frebinger und raimund.

Frediuger (ieţt fidc) an bas Bett jeiner Fran uno beginnt). Arfitern, Mariedl, wie geht's bir? Schlecht, fagit, is net zum glauben, ichauit ja jo gut aus. Mirit bald

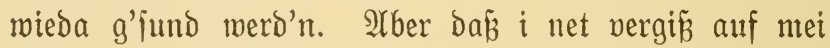
Red! Der Ioftor ift ba g'mejt, bu hait a Scbrift auf= jeben laijen?

Frau Fredinger (ipöttij(h). Du meinjt wegen bem Tejtament?

Frediuger (einen icheuen Blidf auf fie merfend, um bann um jo liebevolfer jortzufahren). Na jo, Maried, wenn bu's ichon fo nennen willft. Der Doftor hat mir ef' fo was ähnliches beim 'nausgef'n g'jagt. (Seine Inngeoulo ichwer bezägmend.) SUllio hajt du es wirflich g'macht?

Frau Frediutger (rubig aber entjobieden). $\Im a !$

Frediuger (auffahrend). Ja? Was joll das heiß̄en? Du willit aljo bie Rinder zu Erben einieben?

Fran Frediuger (ichwer atntend). Sa, denn von bir friegen's ef' nichts! -

Fredoinger (fich bemühent recht liebevoll zu fein, währento er 
fanm jeinen Born bemeiitem fann). Itber Maried, ichau, jeb̧t find wir fo lang werheirat', du hajt alles 'friegt majt mollen hajt.

Frau Fredither. Das billoejt bu bir ein.

Fredinger. Na, hait bu etwa net alles befommen und die Sinder (bifitig und böhnend), die lieben, guten finder, hab' ich ihnen nicht alles geben was braudft bab'n?

Frau Frediuger (gedelynt fragent). Meinen Rindern? Du meinit mohl bie Rinder, bie bu mit ber Cilli und der Sathi g'babt haît? -

Fredinger (mütend, will auffahren, beherridgt jich) jebod noch, nimmt bie Santo jeiner frau, welche fie ihm entziehen will, und itreichelt und liebfoit iie). Stber Mariedl, wer wird benn jo grauplich jein. Mia, na, Marieol, bei Scanderl fannjt mir do no laijen. (Saält ihre heand fejt, welche fie wiederholt vergebliche \$erjuche madgt, ihm zu entziefen.) Es hat a mal a 3eit geb'n, mo's ou mir bie Şano gern laij'n haịt und fönnt's denn net wieder fo wer'n. 2lber bie Rinder

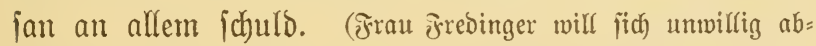
wenden, er hält aber ifge şand feit.) W̧as follten benn bie Sinder mit bem Şaus machen? Wann'ît es aber mir verichreibit, i bin ba Bater und merb' für fie forgen.

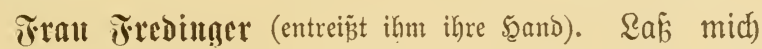

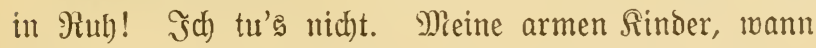
bie nicht einmal bas befommen bon mir!

Fredinger. 2tber mir g'hört's!

Frau Fredinger. Das ift mein Eigentum. Jch hab es mir verbient!

Fredinger. Miteinander! 


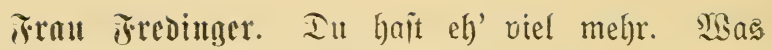
(ic) bab' ijt eh' a Bagatell.

irredinger (mit zorniger Stimme). Illio willit nicht, Marie?

Frau Frrdinger (mübe uno ängitlich). Siein, jeb̧t la mi(h) in $\Re$ iuth!

Miniunund (fommt gin uno frägt). MBas willit denn, 9)intter?

Frredunger (barich). Siser hat bich Denn g'rufen? Schau, dâj d' weiter fommit.

Maiumut. Эach habe geglaubt, die Miutter habe mich) gerufen.

Frau Frediuger. Bleib mur da, 9iaimund. (3u

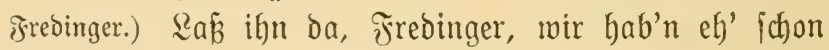
ausg'reot.

Fredinger (zornig). Schau, bá̉ bu meiter fommit, $i$ bin no net ferti! (अaimumb will jögerno weggehen.) Slllio

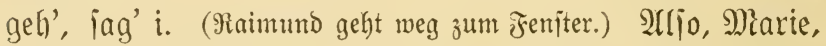
net wahr, ou änderit bas Ieftament und febst mi jum Erben ein?

Frau Fredinger (müde). Sefier' mich nicht, Frebinger, ich) tu's nicht.

Frediunger (mütend, mit gesämpiter, gepres̄ter @timme).

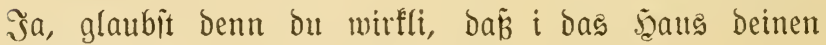

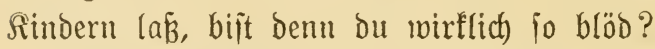

Frrau Fredinger (mit entifeţt aufgeriffenen 2(ugen, jïc) auf einen $\mathfrak{A} r \mathrm{rm}$ jtütsend und halb aufridtend). Mas, Fredinger, bu willit ben findern mein saus itreitig machen?

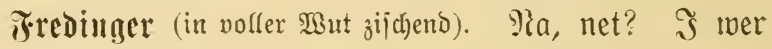
Deinen Banferten das Szaus laîten? 
Tran Frodinger (entiest ausrujent). Sejus Miaria uno Solef! Banferten meine Sinder, So a idslechter

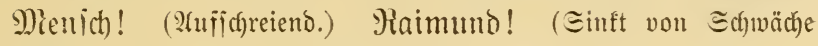
befaflen zutücf, reijer.) Haimuno!

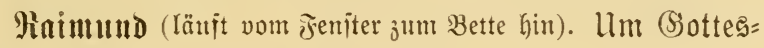
willen, Miutter, was ift bir Denn?

Frall Fredinger (itojweife atmeno). Mitr iit... ichlećt . . Haimumo . . Der Şater . . Der Water!

Matumund (zornig zu fresinger). Du bajt ibr wae tan.

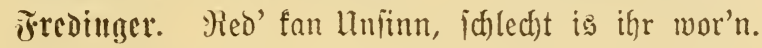

Frul Fredinger (idjer atmend). Mir is ichlecht... Yiainumb

Raimund (erichrofert). Miutter!

Trau Fredinger. $\Im$ itirb'...

Miaimund (halb weinerliden Ioneś). Miutter! (Zaujt

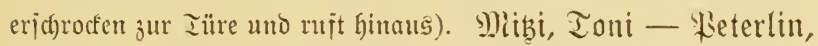
hol'ns den Hepi; der Matter is ichlechter!

\section{Beunte Sjene.}

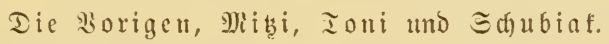

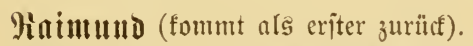

Whipi, Toui und Sdjubint (itürzen Ginter ihm herein uno rufen). Mas ijt Dem g'icheh'n?! Der Miutter is id)led)ter?

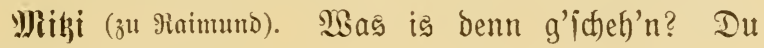
warit ja herimnen?

Minimuı. Ter Bater hat mit der 'Mutter g'ïtritt'n und --

Fredinger (zornig unterbrechent). Schlecht is ihr wor'n uns ba hat's an Anfall friagt, fie wirb fie idfo wieder Dajangan! 


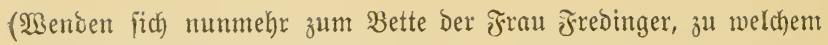
Ioni bereits geeilt ift und alle ftehen neben bem Bette.)

Toni. Mutter, was is bir Denn?

Frau Frediuger (îterbend). Finder ... mit mir

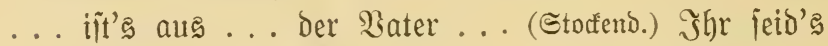
... verjorgt... es fann ench ... nichts geichehn... lebt's wohl! ... lebt's wohl . . (S)ott ichüb' euch! . . . (ঔärlt fraftlos zurüc und fitirbt.)

Miłki uno Ioni (vergieß̃en einige Tränen uno wehflagen). Mutter! Mutter! Bleib bei uns!

Maimund (wild aufiftreiend). Mutter! Mei Mutter ijt tot!

Sdjubiaf (högniich zur Seite). Sa endlid)! Der Allte icheint fie befördert zu haben.

Fredinger (itegt vollfommen gefühllos dabei und fagt beipeite). Umionit! Umionit hab ich mich io ang'îtrengt, na (ballt veritecft bie Fauit) jebst gilt's.

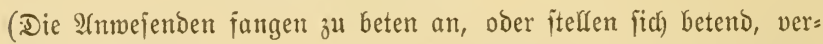
Gängen bie Spiegel uno treffien alle Maj̄nahmen, wie joldhe nach dem eingetretenem Iode ïblich findo.)

Piaimund (zornig und verzmeifelt zu șrebinger). Iu bift

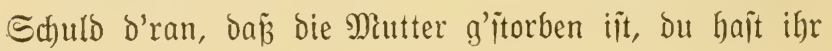
mas 'tan.

Fredinger (grob). Şalt's Maull und red net fo blöb baher.

Mitji (zu Freoinger). Er lagt aber, bu haît g'îtritten mit ifr.

Miaimund. Sa und bie Mutter hat momentan auf= g'idhrie'n unt hat mich g'rufen.

Fredinger (roh). Meil's an Srampfanfall friagt hat, do fann do $i$ nir bafür! 
Erdubial (bei Seite). Şch möd)te wetten, ba hat Der Alte bie 5̃and im Spiele. -

Maimund (zornig zu orrebinger). Die M)iutter hat ja noch was reden woll'n, hat aber nid)t mehr fömnen.

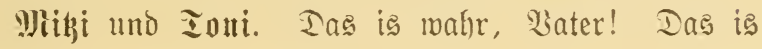
wabr!

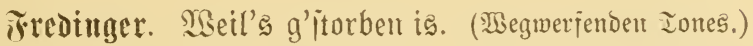
Mas wer $i$ mi weiter mit io an Buab'n 'rumitreit'n.

Maimund (ïeft srrebinger wüten an). Ias werben wir ichon noch ief'n!

Frrdituger (will ifm eine $2(n$ twort geben, überlegt es fich),

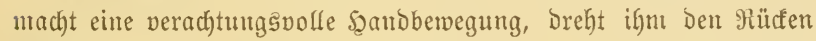

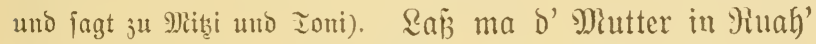
und geh'n ma ins Speiszimmer, bort wer'n ma alles weg'n Der Reich' bereden. (2[Is bieje zägern, brängt er.)

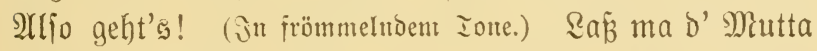
rulh'n, ite is jebt beijer o'ran wie wir. Der serrgott hab's jelig. (Macht plump von ber šerne das Beichen bes Srenzes über bie Reiche.)

(Die übrigen $\mathfrak{Q}$ (nwejenden madben ę ebenjo, verfolgen aber mi $\bar{B}=$

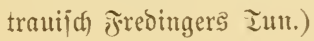

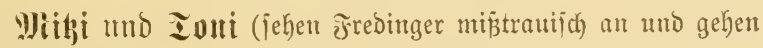
erīt auf Schubiafs Bureben langiam hinaus).

Matulund (miberwilfig, will jich nicht entfernert, worauf Frebinger ungeburbig mit bem jūpe ftampft).

Frreinger. Arljo geh'ı ma!

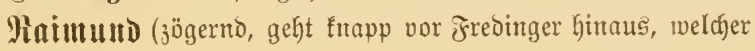
als ber Rethte folgt.

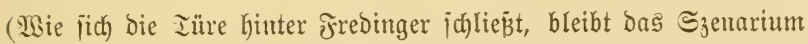
vollfonmen vereinjamt. Man ljört j̧resinger jagen) 
Tredinger. (Siegt's nur. An Mroment glei bin $i$ mieda da. (Uno furz baranf öfïnet fïc) wieder die mittlere ₹üre und jrebinger cricheint.)

\section{3ehnte ভjene.}

Frebinger.

Frrediuger (wirft einen icheuen Blicf anf bie seid)e, läıjt jofort anf Den Rajten von jeiner veritorbenen ortan zh, will ihn

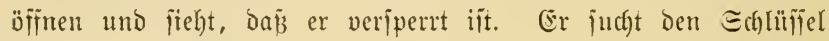

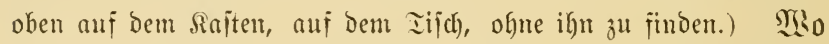
ijt benn unr der Echliilficl? (\&äuft zum andern siaiten.)

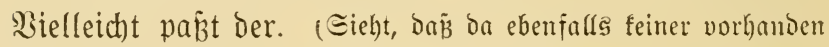

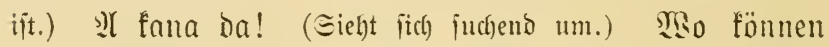
mur bie Edylïfifel fein? (9iad)benflich.) Eoll's am End' ane von Den Mabeln hab'n, ober (mirft einen fcheuen Blicf auf bie seidje feiner Frau) gar mei frau hab'n? $⿴ 囗 t) e r$ wo? (Siel)t mi bas Machtfäitthen hin.) $\mathfrak{H} m$ Machtfaitl mern's

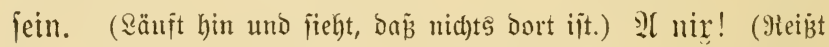

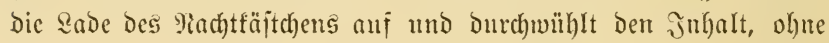
das (sejucl)te ju finder. EEnttänjogt.) Do a nei? (Etarrt einen Moment vor fịch hin, fährt bann mit bem alsgeitresten

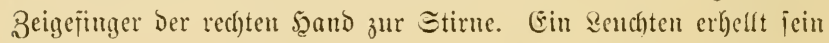
(Beficht.) In fann's mur mei Ţrau im Böriel unterm Sopfoliter ham! (Sieht feine tote joran an, itreft bie jände

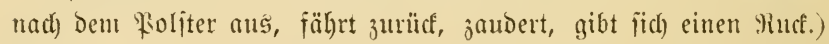

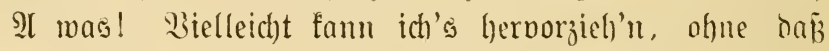
i's anrïl)r'. (3u jidf ferbit; verjucht mit ber linfen saand vor= jitchtig unter ben Foliter zu fahren, fieht, baj es nirght geht; firch

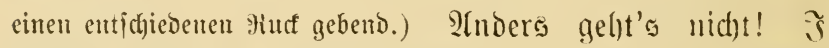

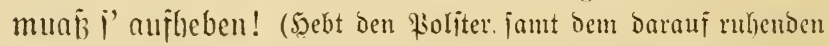
Sopf uno Dberförper feiner Frau in bie 5ähe, uno zieht bie biöre 
hervor.) Mabit Maried, i tua sir ja nir, i bin bir

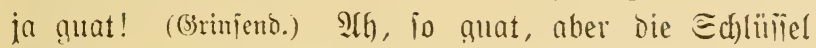

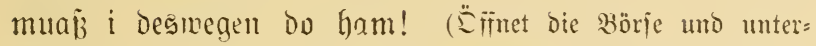
jucht ihren Inthalt, entnimmt berjelben melfrere Banfnoten uno jagt böbniicl.) St paar 3ehuer, io, oe wer ma auffeb'n.

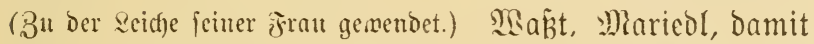
fie dir net verlor'n geh'n, (hähnija) uno die Rinder, bie liaben, guaten finder, de brauchen's net, de hab'n eb' g'erbt. (Brimiend und höhniịch.) 5a, ha, bas hafit, wann's Das Erbteil friag'n. SBapt, Maried, i bin fo biel a füriorạlicher Mienlich) für Deine finder! (5at bie Bant=

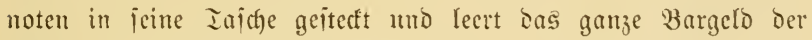

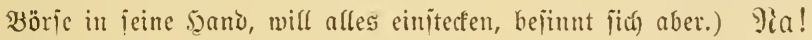

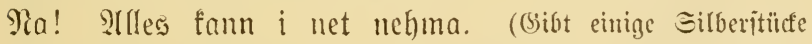

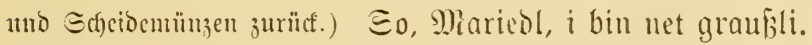
Da hajt a paar Eechierln j'rucf. Mehr brauchjit net für die Jiai' ins senfieits. (Entichieden.) Emuit funnt ma vielleicht gar lag'n, i hab dir dei (Selo g'itohl'n. Iher nie Echliijiel? Ia fühl' i was l)artes im Exitch=

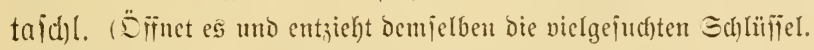
(Brinjend lächelno.) Yia, da häı' ich's ja! sèbt mer'n mer u:a dell $\Xi$ chmudf a hol'n! Plber j'erit (idjiebt bie Börie vorifichtig unter ben Woljter) z'rüctlegen, Damit's niemand merft, Daun iunit funnt ma lag'n, i hätt's (Ssels

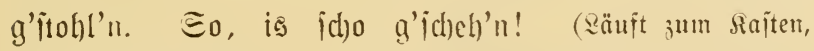
probiert bie Schlüfifel, ipertt auf unb burchitöbert denjelben und bie

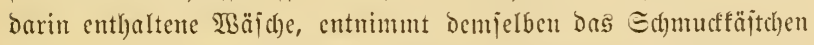
mit Setten, Ringe, Shrringe, S(rmbano. 5ögnemo.) Da. ha, ihren Echındf, den muaß̉ $i$ ạlei auf die Eeit'n räumen, Damit ifn bie finder net friag'n. Sit zmar nid)t viel! Stber i war immer fo g'icheiot uno hab' meiner frau 
immer viel weniger geb'n, als was braucht hat und no baju an Echmuct, io a \&urus! Ien hat fie fich als belcheidene Frau immer jelber faufen milafien. (Wendet

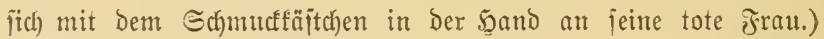
Mgas, Maried, bei mir hajt es immer fo guat g'babt, Daß̉ ou mir als Danf bafür den Echmudf idhenfen faunit. Du brauchit'n jeşt ja eh' net mehr uno bie Banferten derf'n 'n net friag'n. Strer i Maried, i fann ihn guat brauch'n. (5̧at unterbefien bas ভchmunffäītchen geöfinnet, entnimm demielben eine goldene Şalšfette, hebt fie empor und betradytet jie.) $\mathfrak{A h}$, bie goldene Şalsfetten ganz ichön

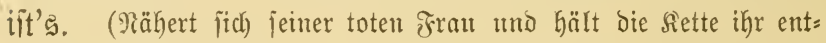
gegen, als ob er jie ing zeigen wollte.) Dei şalsfetten, Marieol, (grinit böhriifa), na, wer glaubit Denn, Daj friag'n mirb?

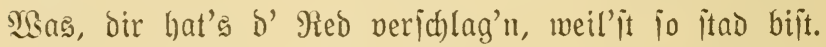
Ia muaí i bir's icho ielber lag'n. Tia, wer foll's denn junit friag'n, als mia dei Freundin, bie Cilli, bie veroient's, bie hat von mir fünf Rinder und viere bavon hat's ichon begraben. De hat auf mein Mat g'hört und hat bazu g'ichaut, ba bie finderln (frönmelno) a beizeiten als Engerln in jimmel fommen, beim fünften ît's nimmer gangen, bös war zu g'iuno. Das is lyalt

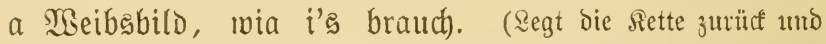
entnimmt bafür bie Dhrringe.) Uno bie Dhrringerlı, dö friagt bie Siati, bie hat a brei lebendige finder von mir, aber fie halt zu mir. (尺egt bie Dhrringe jurinf uno

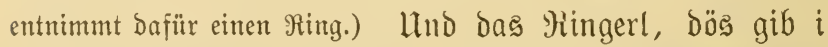
Der Sautsmaiterin, Denn mit Dera hab' i glüctlichermeife tane finder, ab fie is a Ferl'. (Sanz anders als mie mei Frau, dö dumme Pocfen. SBill ifr (Sieló ben 
Findern vererb'n. (5̧at Den ring zurüd gelegt und bas

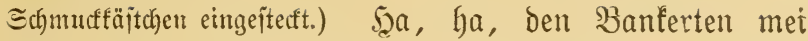
(Seld! Sa, baber! (Macht mit bent Daumen die entiprecthende Bewegung.) Pichts derfien's friag'n! J゙h hab' icho vor=

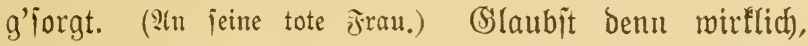

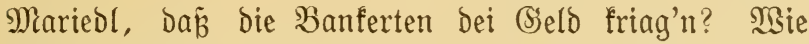
fann ma denn nur io blöd jein, du dumme lurjed'l! Das wiro a \$rozé wer'n! Pia, eing'fabelt ham' mer's guat, benn mit (Selo fann ma alles mach'n, bös ras i am beiten. Yrber war das notmendi? Jeätt's mir net bas (Seld verichreiben fönnen? So aber muás $i$ do no an \$rojez führen? § funnt mi frunım ärgern! (अiebet jich immer in größ̄ere $\mathfrak{B} u t$ Ginein. $3 \mathfrak{u}$ jeiner toten Frau.) $\mathfrak{A m}$ liabiten hautert $\mathfrak{i}$ bir no ani abi. (Shebt bie Szand. wiitento.) IU blöbe STocfen. (Schimpft unflätig.)

Maimutud (ipringt Gerein).

\section{Elfte હzente.}

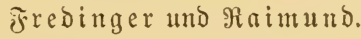

Miaimumo. Miüfre' bich nicht!

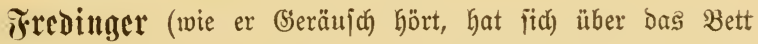

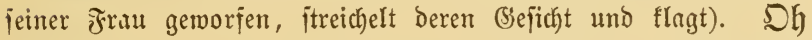
mei Maried!! mei arme, arme Mariedl! (Wie er bes

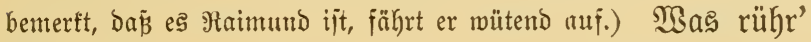
bich nicht?

Maimund (iït ipracblos ïtefengeblieben und idgreit bann 3ornig). Du hajt bie tote Mutter idllagen wollen?

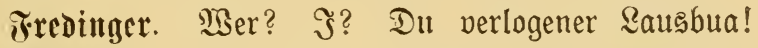

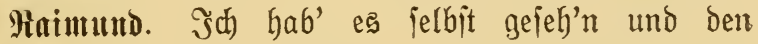
હch)muct hait aud eingeitecft!

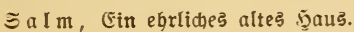


Trediuger (fährt wütenb auf, als er fich) entbect fieght und

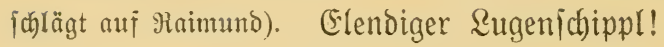

Minimumd (totenbleidh, will zurürfichlagen, befinut jid jeboct,

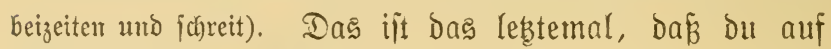
mich geid)lagen hait, Water!

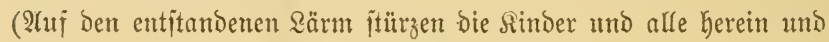
fragen Durcheinander.)

Mlle. Masas ift benn geichehen?! Masas hat's benn geben?

\section{3wölfte 巨zene.}

Die Borigen und Mitzi, Toni und Sanbiaf.

Minimumb (mild). Sluf bie Mutter hat er id)lag'n woll'n. Den ङchmud hat er aus'n Siajten genommen und eingeitectit.

Aflle (burcheinander, eritaunt). Mgas, ben Schmudf? (Sehen auf den nod) offen ïtehenden Siaiten. Miţi, Ioni uno Schubiaf Iaufen bin uno fehen nad) uno da fie bas Fehlen des Schmudfes entbecfen, itürzen fie zurüct zu șrebinger, ber mittlerwcile Den ßerjuch machte, davonzueilen, und itellen Fïh ifm entgegent.)

Erjubiaf (gierig iajreieno). Schuiegerpapa, ben Schmudt müifen Sie zurïffgeben, der gehört zur Berlafifenichafts= maije.

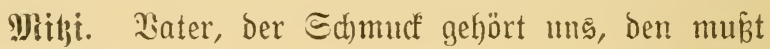
Dil hergeben.

Toni (befräftigeno). Sa! uns gehört er!

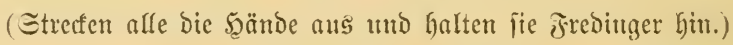

Fredinger (wild). Was, euch? In Iredf g'bört eud)!

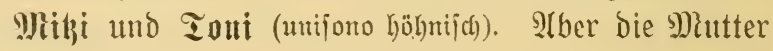
hat $u$ ins alles vererbt. 
Jredinger (müteno). Die Erbichaft habt's ifgr no net. Die wir $i \cdot e u d$ no orndli verjalzen.

Galubiaf. Shruiegerpapa, bas ift Diebitahl; ment bu ifn nicht zurïcfibibit, mach) ith bie 2 nzzeige.

Fredinger (fiefyt fïch) von affen Seiten bedrängt uno feinen

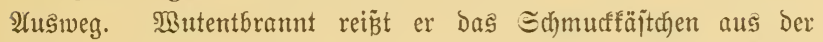

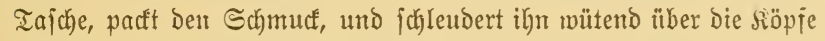
feiner ihn bebrängenden Familienmitglieber und gegen bie seiche feiter Frau und ichreit). Da habt's den Drect!

Toni, Mithi und Edjubiaf (iehen bies erforectit und

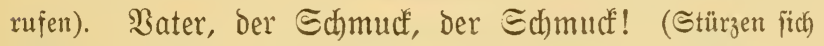

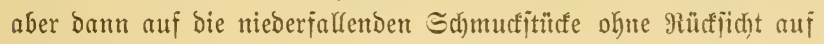
bie Reictye.)

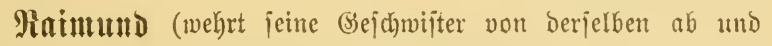
jtellt jich mit ausgebreiteten S(rmen vor biejelbe).

Frediuger (ïid) an Raimuno wendent mit brohent anfge= hobener 5and, farreiento). Da jiebjt, mas d' ang'richt hait. Mart, Rausbua, bas wirit mir zahlen. (\$(b.)

\section{Dreizebinte Szene.}

Die 3origen ohne Frebinger.

Mitji. Ma, fowas! Şaut den Schmucf ba her.

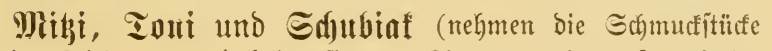
von ber Reiche meg umb heben fie vom Bimmerteppich auf, unb be: trachten biefelben prinfend).

Toni. Piichts ijt ifm g'icheh'n!

Mithi. Das war noch a (Sslürf́, Dá̉ er baher g'fallen is, ftatt auf ben Boden.

Edjubinf. Denn hätt' er tie auf den Boden ge= worfen, fo wären fie bejhäbigt worden, fo aber find fie 
weid) anfgefallen. Die liebe Schwiegermama hält's ja gut aus. (ફ̧ähnifch lächeltro.)

Minimund (ber inzwijchen mit jeinem zajchentudhe bie burd) Miederfallen eines Schmucitiürfes im (sefichte feiner veritorbenen

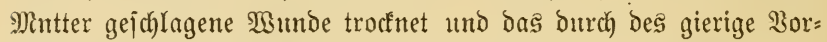

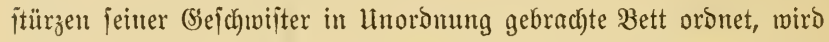
mun aufmerfiam, fäbrt entiekzt auj uno ruft Schubiaf zu). So was zu \{agen, ou bift ein niederträchtiger fierl!

Equıbiaf (frech). গুBas? Niederträchtig ichimpfit bu mich), ou frecher Rausbub! Sch mur bir boch ein= mal eine geben.

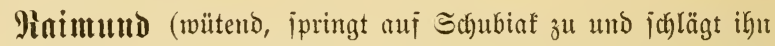
mit gebalfter Fauit ins (Beficht). Du Şaderlump! Mir willfit ou eine geben?

\section{Mierzehnte Sjente.}

Die 3 origen mo sepi.

Mithi, Toni und Ed)ubiat (wollen fid) um fojmpjend auf Miainumb fürzzen. Sn biejem Miontent tritt \$epi ein, jtutht,

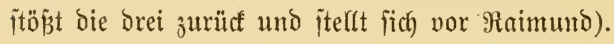

Hepi. Raß̧t's mir den Raimumo in Muh'. Er hat ganj recht gebabt. Schamt's euch net? Die Mutter ijt no net a mal recht tot. Sdyaut's lieber, baḱ neiter fommt'e, fonit . . . (Mad)t eine brohenbe seberde mit jeinen şäıı̈ter.)

Mitpi, Toni und Safubiaf (ichauen jich veroukst an).

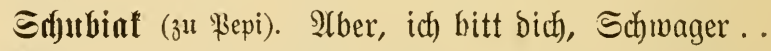

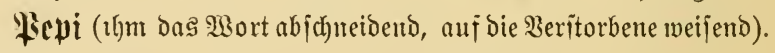
je id)on recht. (Seht's, laß̧t's die Miutter ruh'n.

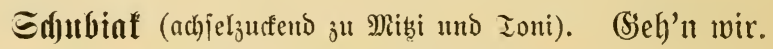

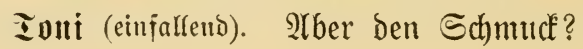


Mithi. Den nebmen roir mit uno bamit ifn ber Bater net wieder nehmen fann, werden wir ifn jelber aufheben. (Iachen jid) gegenjeitig verittänonisinnig zu uno gehen binats.)

\section{Fün†̇zhnte ङjene.}

Raimund und Pepi.

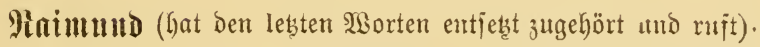
Uno das find meine (Sejchwijter. (Betrachtet verzmeifert feine tote Mutter unt ruft.) Mutter, ou gute, arme Mutter!

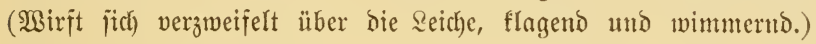
Mutter, meine Mutter!

Pepi (ïteft mit gefalteten Şänden, ein leiies Bebet mutrmelno, neben ber Reiche. Rach einer $\mathfrak{B}_{e}$ eile legt er feinem von Schnterze

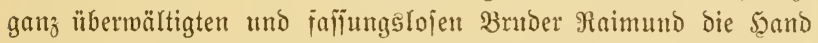

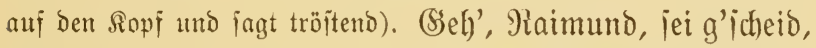

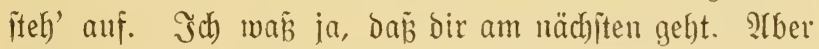
unier serrgott hat's ichon io iodl'n.

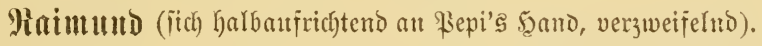

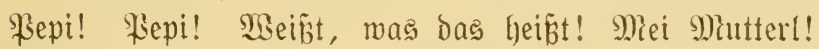

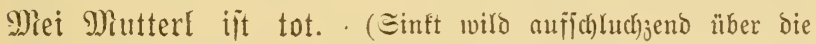
Seiche, morant Fepi mit Tränen in Den S(fugen fagt.)

Psepi. Armer Sierl! Mit ber Mituter ift ifym jei Mselt g'ĩtorb'n. Strmer B̉ua!

(ख̂orhang fällt.)

Eีd bes eriten Afteś. 


\section{Bureitev dat.}

Frebingers Eomptoir.

(Semöhnlich, untrein, vernachläjfïgt. Dinfs feitmärts ein alter Schreibtijd). 2(n Der gegenïberliegenden $\mathfrak{W a n d}$ in ber Ëfe bie eijerne

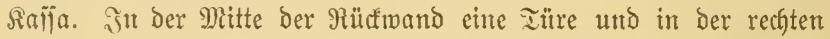
Seitenwand ebenfalls eine Türe $i c$.

\section{Srite Şљne.}

Fredinger.

Frrediuger (iteht vor Dent Spiegel, befieht fich) von oben bis unten und liebäugelt mit fich felbit). Sla aljo, jebt wären mer Mittiber? 5ृa, MBittiber! Jeb̧t tönnen mir tuan und mad)'n was mir moll'n! (Wiederbolt in fragendem Tone.)

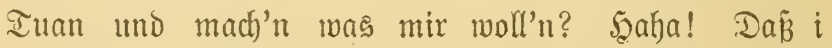
net lach', als mann $i$ net immer tan bätt, wos $i$ bob woll'n. (F̈ängt laut höhniflc) zul lachen an, exjchricft ob beঙ

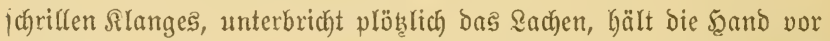

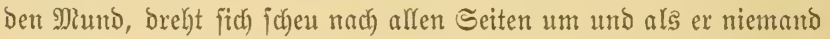
jiebt und nichts verbächtiges hört, jagt er leife wie ju jich jelbit.) $\mathfrak{U}$ ), laut lacken berf $i$ net, funit manerten meine Reut,

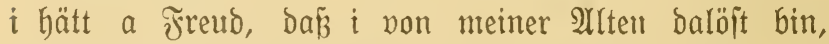
aber was i net fag', dafj mei gottjelige Frau (mit Gimmelwärts gerichteten 2(ugen uno fröntmlerifchen (Bejtcht) ge=

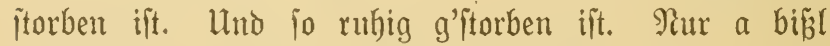
z'ichnell ober eigentlich) a bif́l z'langiam. İ hab zwar 


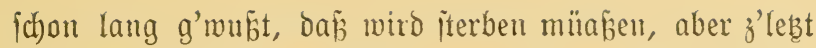
is ma z'fdunell gang'n. Mann's aber no idneller gang'n wär', war's mir no liaber g'meit, Denn bann hätt's das Iejtament net fo mach'n fönna uno mann's langiamer g'meit war, fo hätt i's do no bajua bracht,

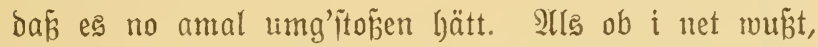
dañ alles geft, no baju mit an Schüppel (Sseld. Stber das hätt mir halt ber Doftor genau fag'n foll'n, wann's eigentli itirbt. §s a a bummer Sierl, denn er hat

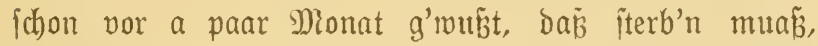
hat mir's a g'iagt, aber er hat mir net jag'n fönna bis mann's eigentli iterben wird. Es muís alio do wahr fein wia's g'bajen hat, ber Doftor halt zu meiner Jrau, aber weil $i$ joviel an ehrlicher Menich und und Charafter bin und a Sa and bie andere wajht (ntadt mit Den Şänden bie entiprechende ßemegung) ma glaubert net, was io a papierene Saf' alles fann, hat er eigentli ful mir g'halten, natürli nur weil $i$ joviel an ehrlicher Menich uno a Charafter bin uno man iich auf mi verlafijen

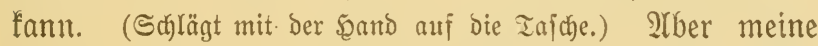
Fintor hab'n was g'neift und hab'n an andern Doftor und extra an \$rofeĩor hol'n lafi'n, (Gögnifa) als ob's de ianeller und bejfer hätten madjen finna. Ta -

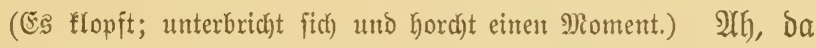

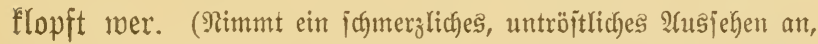

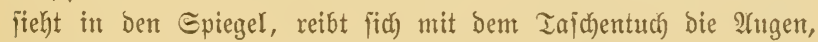
bamit fie rïch röten.) So jeb̧t fitimmt's! Szerein!

(Peterlin tritt ein.) 


\section{3meite $\Xi_{j}$ ene.}

Fredinger und seterlin.

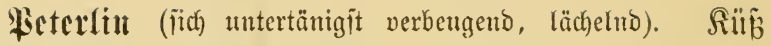
d’ Şand, gnä Şerr!

Fredittger (wie er fie fieft, ninmt er eine ungejmungene Sraltung ein und geht ihr neugierig zwei bis brei Schritte entgegen). MBas is, Feterlin? Bringan's was? Ş was g'weit?

Fetertin. Sa, gnä Şerr! (Greift unter ihre Schürže, Golt einen Brief hervor und gibt benjelben, fich vorfictstig um=

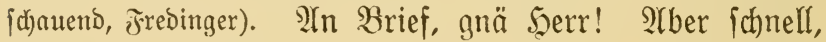
gnä Seerr, idhelll! Die junge Frau hat g'jagt $i$ joll ihn refommanbiert aufgeb'n an ben Dr. Seger und glet z'rucffommen.

Fredinger (erregt, reiß̄t ihr den Brief ans der Şand und liejt bie A(brefie). Scerrn Dr. Seger. (Radht grinjent.) Sapperment an Dr. Jeger, bös geht mi an. (\&aüt zu Türen und verriegelt betDe.)

Picterlitu (brängeno). Ed)nell, gnä Şerr! ¿̌ bitt Şna, gnä Şerr, tummeln's Ş)na!

Fredinger (greift in bie Iajche, gibt ihr einige (Suldoen). Da hab'ns, P̉eterlin. Sö mers'n Şha icho aubaliag'n.

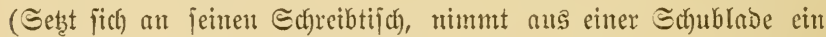

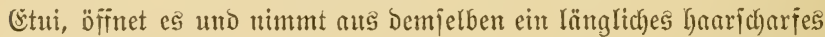

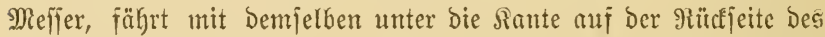

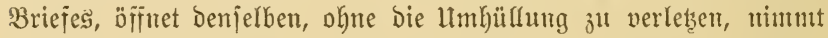

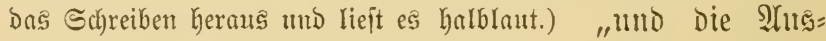
fage, melche bie \$seterlin abgelegt hat, iit falich)." (Zornig, laut.) N⿴囗十art, farnalli, i mir Dir's zeig'n. Wieine Reut

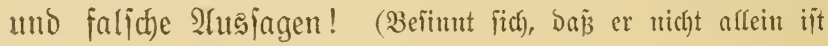




\section{$-41-$}

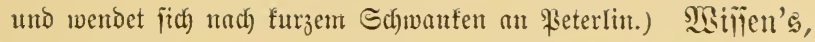
was bie Ioni fareibt und no bazu über Şhna?

Heterliu. İber mi?

Fredinger. Daß̧ $\Xi_{e}$ in bem Erbichaftsprozé falich ausg'iagt hab'n.

Fieterlin (erbleicht und jagt fieberno). $\Im$ !? Smo jteft Delln das, gnä Şerr?

Frebinger (zeigt auf bie Stelle bes 3riefes). Da Der.

Peterlin (lieît und jagt bann). So was, als ob $i$ lüg'n föunt! So an ordentliche Fierion wia $i$ bin! Falich ausg'iagt foll $i$ hab'n. Is ja net möglich, benu i hab ja nur das g'iagt, was mir ber gnä Şerr vor=

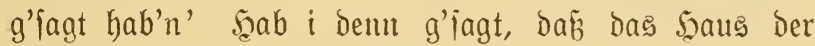
veritorbenen gnä Fraul g'bört hat?

Frroinger (zornig). Sö alte llrichl! Das fönnen's gar net g'fagt hab'n, benn bam hätten's ja gar net

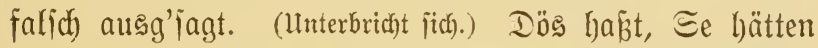

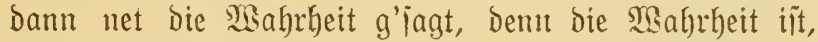

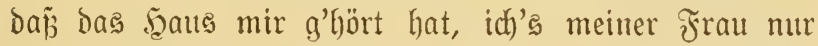
proviforifch) verid)rieben hab. (Etellt iid) in Foje mit bes fdhwörender, empört fein forfender Stimme.) Szeilig iit bas Eigentum!

Beterlin (zur Seite). Beponders wann's fan andern g'hört!

Frediutger. Jjab'tis was g'jagt?

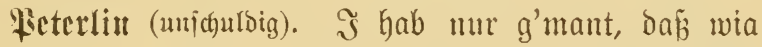
Der gnä Б̄err g'rufen hab'n: Şeilig ift bas Eigentum!

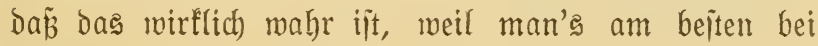
Shnen, gnä Şerr, fieft.

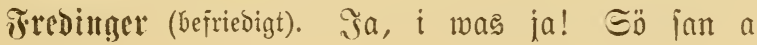


rare Fraut, anf die $i$ mi verlaijen fann und de zu mir halt, Denn $i$ bin fowiel an efrlicher Menich und Sharafter uno mei saus is an ehrliches sauts, an altes 5ૃalls!

Weterlin (brängeno). Den Brief, gnä Seerr! Sonjit hab i Berorun. Die junge Frau is feit a paar Iag eb' fo ipieß̧i auf mi. Sest mas $i$ a marum?

Fredinger. (S)lei, \$eterlin, glei! (Stectt bas Echreiben

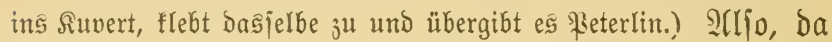
bab'ns den Brief z'ruct. Plichts ift ifm g'icheh'n. I hab nir g'iefn uno Sö a net. (Mit dem ginger brohent.) Beritengan's?

Peterlin. Ulber gnä Seerr! § wer bo net $a$ fo an guaten und liab'n Şerrn verraten?

Frevinger. Sur immer guat auppafien uno wann's was fef'n, hör'n ober in b' Şand friag'n, mur glei

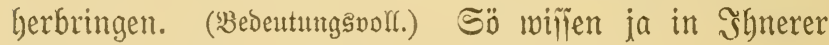
M̉ohnung (gebefnt, hinterlijtig) dö Sacherln.

Heterlin. 2rber, gnä Şerr, alles, alles was nur soll'n.

Jredinger. Und bann, $\subseteq_{0}$ wififen ja eh'. $\Im$ bin net $\{$ d mmutici.

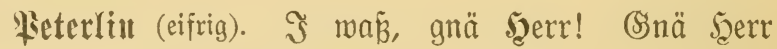
hab'n io a guate offene Şand. Sinä Şerr, i -

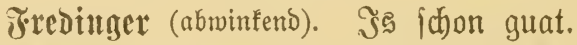

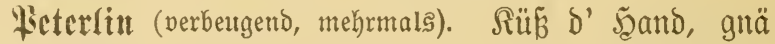

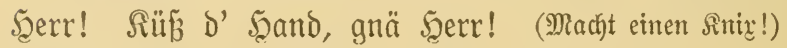

Fredinger (nicft ifr z̧u). 2roje.

(\$eterlin ab.) 


\section{$-43-$}

\section{Iritte Sjente.}

jrebinger.

Jredoinger (ifre nachiegens). Sarnalli, Ruber, io an elendiges Mseibsbilb, itiehlt wia a Yab, was ifr unter ঠ' Sand fonmt, liagt, betrüagt too's und wia's nur

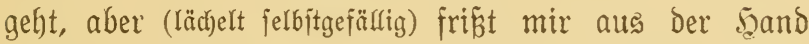
und tuat alles was $i$ von ifr verlang. Denn eritens (verítänonisinnig) lunnt i's einipirren laijen und zreitens lá̧ $i$ ifgr was ertra verbiena uno (mit Göhuijcher ßetonung) auf jolche Reut hat fi mei Frau verlaif'n und hat g'laubt, fie hat a ebrliche Ferion an ihr, (grinjent) babei hat's mir alles verraten. $\Im$ bab oft ichon g'wußst, was mei Frau und meine Rinder tuan wer'n, bebor fie's no tan bab'n. Sa, ja! Die Ehrlichlet mit der is a eig'nes (S') frett. S fag halt immer, efrlich fam a jeder Dumme Sierl fein! Pa und bab i net recht! Ias is grod fo, wie mit bie গ̂lujagen in bem Erbichaftsprozen, ben i gegen meine finder fübr'. Die Ioni ichreift ba

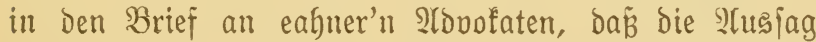
von ber fieterlin falich) ijt. Misia fann man nur jo blöb fein und fo was jag'n, wann man's net berweifen fann? Und wann fie's jelbit beweifen funnten, fo reot' eafina, eahna eigenter Doftor bas wieder aus. SBiefo? Ja, bas ijt a eigene Sach, bas macht alles mei 2rovofat mit ber papieranen Saf'! Der Dr. Scaticheff, (jich unter=

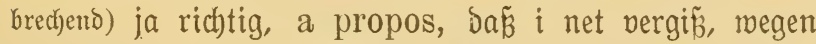
ber Slusjag von ber Cilli, bie is ja für heunt' vor= g'laden, mit ber mú̃ i glei reden. Der Dr. Scatichef hat ma zmar glei telephoniert, bás alles gut gangen ijt, 
aber $\mathrm{i}$ muaß́ do wiijen, was fie g'fagt hat und baj net eppa falich ausg'jagt hat. Dumm gnua is ja bazua, mann $i$ ifr a alles vorg'iagt hab. $\mathfrak{F}$ wir's liaber glei aug̣frag'n. (\&äuft zur Geitentüre uno ruft.) Cillit!

\section{Bicrte $\Xi_{j}$ ene.}

srebinger und ein Schreiber.

(Die Tür an ber Seite geht auf und ein Schreiber tritt herein.)

Sdpreiber. Şerr von Frebinger, bie Fran Cilli ijt nod) nid)t zurürde.

Frediuger. So? Dann muá̉ îe bald mieder da jein? Sisann's fommt, joll's jofort zu mir fommen.

Edjreiber. Bitte, Seerr von Fredinger, merbe es ausrichten. Şerr von Frebinger, ber Şerr Recter ...

Frediutger (unterbrecthent). SBas ift's mit'm Recfer?

Endreiber. 5̧err von Fredinger. Er hat zul Şerrn von Fredinger hineinmollen und ba mar zu und ba hat er bringend g'fragt nach) Jerrn von Fredinger...

Frecdiutger (unterbrechent). Dringend jagen's. (Befiunt

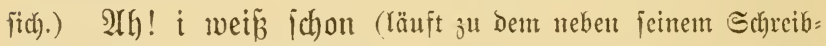

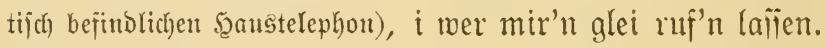

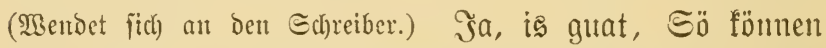
ichon geh'n und bie Cilli joll, wann's z'rürffommt, glei zll mir fommen.

Edretber. Bitte, Seer pon Frebinger.

\section{Fillitte ఠzene. \\ Frediuger.}

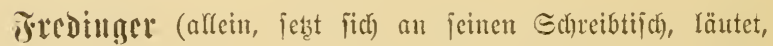

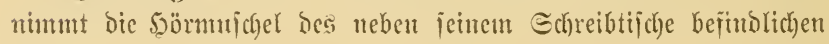




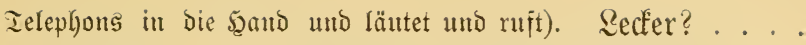
Sa, Secter.... foll jofort ju mir fommen.... (nad) einer $\mathfrak{B}$ eile) fommt gleid)! (Siut! ( aus Der Şand, läıtet ab.) Sa, ber Lecfer! Mag wirb er benn wollen? smann er's ichon io prefifant macht. In Der Fabrif ijit niüts g'ichehn, denn da hätten's mir ichon bie andern g'jagt. Ia fann jich's nur um bie Sdriften handeln, bie wird er mir geben wollen? Эa, Der Lecfer, der Lecfer, wie ber mir hilft, daß̉ i ben Erbjchafteprozeß́ gegen meine Rinder g'vinn. Ia hat mei Doftor, ber Dr. Şatichef, mir g'iagt, es wäre ein gutes Beweismittel, dá̉ das ફ̧aus wirflich mei g'hört, wann g'vilife Edbriften ba wären, aus benen mei Bejīb= tum hervorgängat. Da hat ber Recfer, der net nur eh' ichon geichworen hat, dấ Das Şaus uno alles mei g'yört hat, g'iagt, er verichafft mir bie Edhriften, bie wir brautchen uno er hat \$gort g'halten. Sa, meine Seut jan mir anbänglich), (ielbitgejänlig) hab'n aber a alle Uriach baju. Sag'n ma nur der Recfer z. B. Der is als a ausg'bungerter Maurerg'iell zu mir fommen vor a zmanz'g, fünfazmanz'g Sahrln. Die Sunn' hat rein Durch eahm burchg'icheint, heunt is er a g'machter Mann

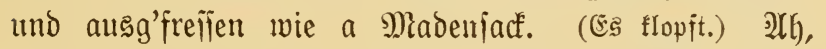
mir icheint, da is er ichon? Allitern herein! (sectertritt ein burd' bie mittlere Türe.)

\section{Siebente Szene.}

Frebinger und acter.

Qedfer. SSuten Mrorgen, Şerr von Frebinger!

Jredinger (niaft mit dem Ropfe). Sö hab'n zll mir eina woll'n? Ş eppa megen bie Sdyriften, oder... 
Secter (unterbrect)end). Эa, Şerr von Fresinger!

Frediuger (ungebuldig). Irlitern dann geben's nur glei her. (Strectit die Şand aus.)

Beffer (framt in ber Eeitentajaje feines giofes herum und ziefyt aus berjelben einen Fad Sdyriften, weldhe er Frebinger gift). Da find's, 5err von Fredinger!

Fredinger (hat biefelben gierig ergriffen unb fieht fie burch). Sehr guat', Recfer, fefyr guat. Arber bas fan net alle? Der Syabler und ber ßonigl fehlen a. SMas is mit benen?

Becfer. Эa, Şerr non Frrebinger, Der Şabler, Der will net recht.

Fredinger. SMarum benn nöt?

Secter. Er hat g'iagt, er muar fie's no überlegen!

Jredinger. Şaben's ifm? (Macht bie entiprechende Obeberde bes Beldozählens mit Daumen und Beigefinger.)

Beffer (nift). §a, aber...

Fredinger (erleidytert). গa alítern!

Secter. Ya, ja, Seerr von Frebinger, aber er mar in msicfel=mactel uno hat g'iagt, er muan fie's no über= legen uno or la jat mir's bis morgen jag'n.

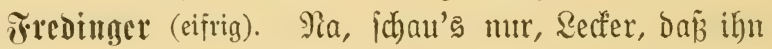
bazubringen. (2iftig.) ऽö lönnen no was zuleg'n, (gebehnt) natürli, nur wann's notwendig ift.

Secter. Das wir $i$ eh tuan mütán'n, denn i hab's ihm fo quafi veriprochen.

Fredinger. $\Xi_{0}$, jo.

Qedfer. Sa ja, damit i ihn mur oazua bring. SMenigitens ben no, denn mit dem \$onigl wirs's abjolut nir, wer'n. 


\section{Frediuger (auffalreno). Marum nöt?}

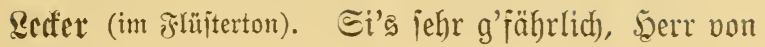
Fredinger!

Jredinger (Feine Unnube Ginter (strobheit verbergent). Atber was reben's benn für an \$amlatich z'iam. (Reife.) Mas g'fährlich?! (Eaut.) M̂ann ma fei Eigentum ver= teibigt! ?o bazu gegen io a Strabanjerbanda, wie meine Rinder jan! Majo? Marum? 5zeilig ijt bas Eigentum! Und i bin a anitändiger Menich uno Eharafter.

Becfer (berufigend). Slber regen's Shna net auf, Seer von Freoinger! $\mathfrak{I}$ hab mur g'mant.

Fredinger (bie gefpielte 2 tufregung ablegent, neugierig brängend). Ra was benu? Reden's, Recter, reden's.

Eceffer (reife). Der Ronigl tuat's abjolut net. -

Frediuger (Iauerno und leije). Marum net? Daben's ihm benn nichts... ober veriprodhen. (Miacht bie ent= iprechende Bemegung mit Daunten und Beigejinger, b. h. Seldo.)

Secfer. Ja, aber er hat mi beinah ausig'ichmifif'n.

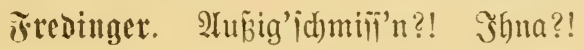

Secter. Sa uno g'idjri'an hat er. SMann's a für'n Scerrn von Fresinger g'hört, io verzicht er liaber auf bie Riefermngen an io an Rundichaft. SBann er a a flaner Ssemerbsmann ijt, fo ijt er immer an elyrlicher, red)tichaffener Mann g'meit und gibt ife net zu aner joldhen Rumperei her. Sia uno (z̈̈gert uno will mit ber Sprache nicht rectgt heransrücfent).

Frroinger (gejpannt und anciferno). Tia und ...

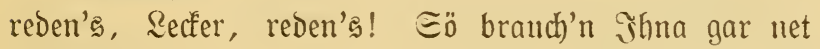
zu genieren. 
Secter (z̈̈gerno). Dumm's Beug hat er z'Famng'reo't. Frredinger (brängent). YUlio jagen's es! (Seije zu zecter.) Recfer, $i$ muán alles wifi'n, damit $i$ wa i mi j'richten hab.

Secter (entichloijen). Er hat halt g'iagt, 5eer von

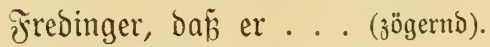

Fredinger. Táz er?!

Seffer. Dá̉ er net mithelfen will, bie Sintor um iłr Erbteil zu betrügen.

Tredinger. Rausbua, orectiger!

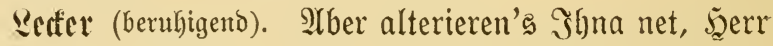
von Frebinger, wegen fo an \$üldher.

Frredinger (begei tert). Sa, a \$iulldher ijt er. Mecht l)ahen's, Secfer, nir als a ßüld)er ijt er, der \&ausbua, ber brectige, und $i$ bin a ehrlicher Menich, uno a (Eharafter. Mas, Recfer, hab i Mecht.

Beffer. Э̌a, Seerr von Fredinger.

Fredinger (neugierig unrubig). Slber hör'ns, Recter, hat er funit toas g'iagt?

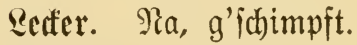

irredinger. Saas benn?

Qecter. Rix b'funders (zögerno).

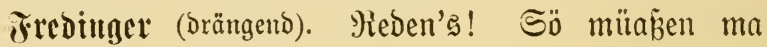
alles jag'n. (Sjeniern's ふ̌bna net, wann er mi a be= leibigt hat. - Mas funnt benn fo an ebrlicher, recht= (c)affener Mienich, wia $i$ bill, pon io an Rausbuab'n, fo an \$ülcher anders derwarten als a Ssemeinheit. Na net?

Beffer (z̋̈gert uno jagt baum entichlojifen). MSann i’s icho jag'n muañ! Er hat halt g'iagt, baí er immer 
nur für bie Frau von Frebinger g'arbeit hat uno nia net für Э̧na und d'rum tuat er besmegen bas a net mit bie Sdfriften. Und...

Jredinger (auffagrento mit geipielter Ėntrüftung). DaS is a Ruag', der liagt, der Rump. Für mi hat er g'arbeit', mei hat bas şaus g'fört und waun $i$ jest bie Redhungen umidreiben lá̉', io gebt's eahm an Drecf an. Na, ber foll mir nur no amal fumma!

Lecfer (unterbrechend). Der fummt Şna net! Der net! Da fönnen's ruhig fein.

Fredinger. \$apperlapap! Sö fennen die Reut' no immer net. Mirb [cho' wer'n!

Secter (entichieden). B̉ei Dem aber net?

Frredunger. 5̧a! ha! SBarum benn g'rab ber net? So a Şungerleiber, ber braucht mi. $\Im$ mir eafm's idjo hamzahl'n!

Redfer (achjelzutfend). Misia's glauben, Şerr von Fredinger. Waann er's nutr net uns hamzahlt! -

Fredinger (unruhig auffahrent). Şamzahl'n mir! Der! So a brectiger Satmgerleider!

Recfer (unterbrechent). Niber a Şantiger? Denn um= fouit hat er net broht?

\section{Fredinger. Droht?!}

Qeffer. Sa broht. Mia i Shna g'jagt hab, hat er mi ja aufitichmeif́nen woll'n uno -

Fredinger. Sa, bas haben's mir aber no net $\delta a=$ zählt. গ্llitern derzähl'ns.

Eecfer. Wie er mir g'iagt hat, daß̉ er nur für bie Frau von Frebinger g'arbeit hat und nia net für Şna, und dañ er bas mit bie Schriften net tuat, hab $i$ ifm 
no zureden woll'n und ihm zum veriprechen ang'fangt; zum ๔chlun bab i's mit bie Sselder probiert, ba is er mia a MSildor auf bie Iür' zuag'bupft, hat's aufg'rifijen

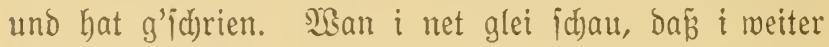

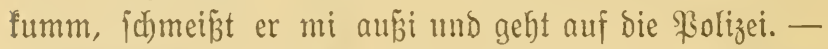

Frebiuger (wie von einer siper geftochen, in bie \$ö̆he

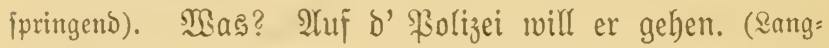
fam wie für fich iprect)end.) Sluf d' Folizei?

Serfer (hebt abwehrent die Şände). 2lber, Scerr von Fredinger, er hat's ja no net tan!

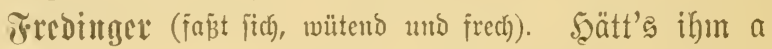
net g'raten. So a Ballot! So a 2ausbua pon a Scungerleider, auf o' Solizei will er geh'n. Mir (ichlägt fich auf bie Bruft). Mir, ben Seerrn von Fredinger, will ber io was antuan uno wegen was? Mseil $i$ mei Eigentum verteibig! und heilig is bas Eigentum.

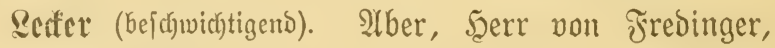
berfangens Şgna do. So a 2Yufregung funnt \$̧na nur ichad'n.

Frediuger. Alber is bemn uet jo?! Sober foll' i's meinen Sindern, ben elendigen Banferten, laî'n? (Jammerno.) Miei elrrlid) erworbenes Eigentum! mei hat alles g'bört! mir allan, a bas Scaus, mas anf mei Frau g'ichrieb'ıt war, g'yört mur mir allan, meiner Fran hat gar nichts g'fört. S(h) hab allan, mur $\mathrm{i}$ allan, bab g'arbeit, mei frau hat g'faulenzt, waun a b' Reut fag'n, mei Frau hätt fie g'racfert Iag uno Pacd)t, uno $i$ hätt g'tumpt uno g'foffen und bätt ben Scerrgott an

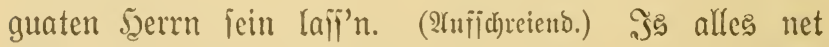
wabr? Denn bie Rent, de fo was jag'n, fan a (5)'jindel. 
Serer hat benn ic idjo was auf an ioldjenten Iritid)= Iratich geb'n? I was am bejten, was wahr is. I allan hab's crmorben! Ditr allan hat alles g'bört!

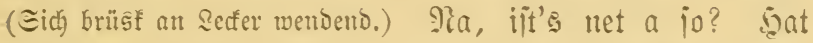
net alles mein g'bört? $\Xi_{0}$ fan lang im Şauts, હö war'n iđjon ba, wia's șaus 'baut wor'n is. હö müap̃en's wilīen. D'rum hab $i$ J̧hna a a J̈mament b'rauf ablegen laijen, daj alles mei g'hört hat. Э̧e ntet jo?

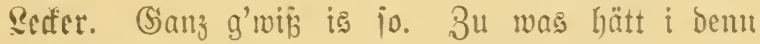
foni g'idyor'n? baj mur Igna allan g'fört hat. -

iredinger. Sia alitern. Iltid ba will io a Etrold) auf d' Rolizet gef'n.

Secfer (unterbrect)ent). Stber, நeer pon Fredinger, er is ja net gangen. $\mathfrak{s}$ bab ja b'rum g'ichaut, baj i weiter fomm', bamit er net vielleid)t in 3orn bo no Ginrennt. - (Berubigeno.) YIber, நerr pon frebinger,

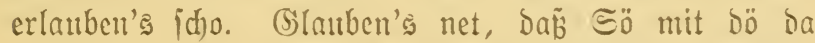

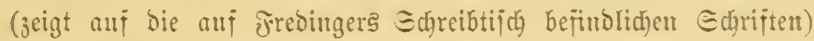
g'nua hab'n wer'n? Biclleidjt giengat 's do?

Fredinger (idwanfend). 5̧m, hm! Pia - - es wirt idjo gef'n. Ier Dr. 5atichef hat mir eh' g'jagt, wie er bie Echriften, dö ভö mir jekst geb'n hab'n, verlangt hat, es möd)t guat jein, wann i's no friag, aber er glaubt, baji, wann's net anders giengert, vielleid)t do allan mit bie eriten @dhriften geh'n wirb, bie ভö mir icho frïher 'bractet' bab'n uno be er Dem Sericht als Bemeisofumente vorg'legt hat. Illitern miro fich's megen be paar lesten, De be brecfigen S3am= fababin net bergeben wollen, a net mehr ipiejen. I 
mir's halt probieren und wir's dem Dr. Şaticher glei

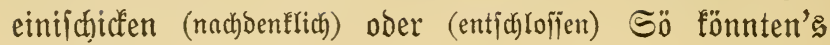

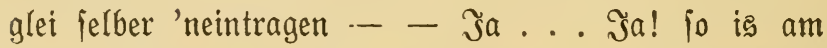
beften. Geh'ns liaber glei jelber eini zum Dr. Scatichef, geb'ns ihm bie Schriften mit aner ichön Empfehlung von mir unb erzähl'ns ifym auf jeden Fall bie (s'; ‘chicht mit bem Ponigl. Ma faun net wiffen und da muáz ma beizeiten an Riegel vorichiab'n. (Bibt bie Schriften dem Recfer zurütc.) Da, da haben's es! Sö miffen, Lecfer, meine Sinder friagen mei (s'jhäft nöt und sö jan ber 2lnzige, ber b'rauf rechnen fann.

(Die Türe öffinet fich uno herein tritt (Cirri.)

Fredinger (bemertft fie fofort und jagt). Du bijt's, Eillli, na wart an Moment. (S)lei bin $i$ fertig mit'm Recfer. (3u secter leife.) Allitern geh'ns nur glei?

Secfer (ïtrahlend). SSlei! glei! Şerr von Fredinger! Dh, wo fich's ums (Ss'ichäft handeln tuat, tua $i$ alles. (\$3auft bie Schriften zujammen ein und will geh'n.) (S'hor= ichamiter Diener, Seerr von Frebinger!

Fredittger (mit ber Şand winfend). Ŝpropos, Sö,

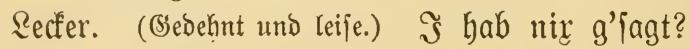

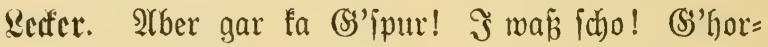
(d)amiter Diener, Seerr bon Fredinger! (Gefta ab.)

Fredinger. Stbje! (Wendet fid fofort an Cirfi.) Эa, was i lag'n mollt, Silli!

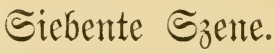

Frebinger und cirri.

Froditger. Der Doftor Satidhef hat mir g'rad früher telephoniert, Daß̉ du guat ausg'iagt hait. - 
(Gilli (beicheiden tuend). I hab' halt tan, was i hab' tuan fönna.

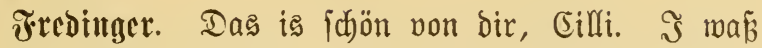
ja, was i für a ßerl' an bir hab'. (2auerno.) Itber ou haft's ja a bejdwor'n? Ret mahr, Cilli?

(Silli (nerlegen). Ja, es is ma eh idher g'nua an= fommen.

Fredinger (ironijd begütigend). NUber was is benn

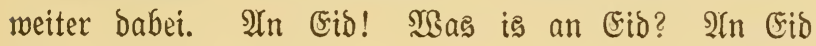
is fünf Sretzer wert! -

(Cilli (will fprechen, fresinger unterbridgt fie). Altber mir Fredinger. Stber $i$ bitt bi, Cifli, es ifit ja nur,

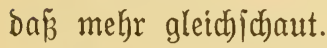

(6illi (eifrig). Ulm das war's mir eben.

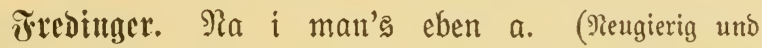

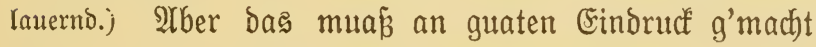
hab'n, wie mei Doftor Dei Beeisigung beantragt hat. -

(silli (unterbrectjend). IYber bas hat er ja gar net verlangt, das heiṕt verlangen fönnen.

Fredinger (eritaunt). Sarum net?

Cilli. Meil's ber andere früher verlangt hat.

Fredinger. Mßer ber andere?

Gilli. Der Doftor pon beine Sinder.

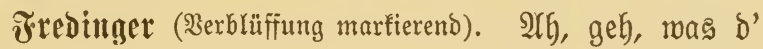
net jagit! ber Dr. Seger?! Ra und wia war benn bie

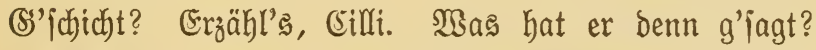

Cilli. Er hat g'jagt, baß żwijchen mir und beiner Familie Freindichaft bejteht und er baher um meine $\mathfrak{S e}$ eidigung bitte. 
Fredinger. AYh! was b' net fogit? Und hat fie Denn mei Doftor net glei g'rüfrt?

(Silli. Ylber ja, er hat ef glei g'idrien: Wiejo?

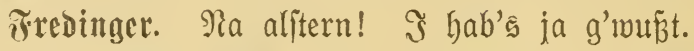

(Silli (unterbrecheno). Saa, aber jeşt hat ber Irdoofat von beine finder zum idhimpfen ang'fangen.

Frediuger. (S)'ichimpft hat er?

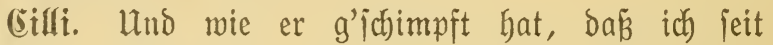
Sałren bei Menich bin und fechs Sinder von bir hob!

Jrebiuger (aufahrenb). So a Rugenidjippl, bas is jo gar net wahr, fünfe war'ns mur, beim jechjiten warit idjon verbeirat.

(Silli. Net wahr und i wir's do am bejten wifijen, bas lechite ift wirflich von mein Mann.

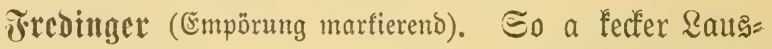
bua! গa, wann $i$ ben amal unter b’ ફ̧äno triag', bem wir i's zagen. Der fann fie g'freuen.

(billi (befriesigt). Sa, zag's ihm nur, den jübijchen Sauferl. I hab' mi eh fo g'ichamt. $2(m$ liabiten mär' $i$ in ben Boben verjunfen. Э hab gar net g'muẽt, dẩ $i$ mi no amal fo icjamen mir müifien.

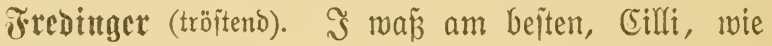
dir g'mejen jein muj̃.

(Cilli (eifriger). Seşt hätt $i$ am liabjten nimmer mehr ausg'iagt, benn $i$ bin bod als ehrliche Ilngeftellte bes Şaujes angeben morden, bie fich ifr 3 rot jeit a oreip̧ig J̌ahren in dein Şaus verbient und quaji zur Familie gerechnet morben is und bie daher am bejten wififen muaj, ob das Szaus dir g'fört ober beiner Fratt. 
Fredinger. Ssanz natürli! So is a.

(iilli. Und wia i glaubt hab', io wie mir's ber Doftor g'jagt hat, er wirb mi fchmören lajīen, um

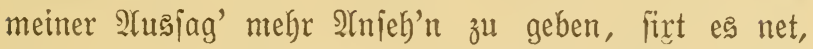
iteht b'r ber Rausbua von an jübifchen Doftor auf und

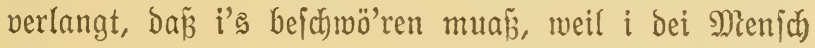
bin und jechs Sinder von bir hab. Sit bas net a (Ses= meinheit?

Frediutger. Und toos für ane no bazu!

(6illi. 2lls of's ifm was angingert, baí $i$ mit bir jects sinder g'babt hab' ober net?

Frediuger. Sanz natürli! das geft eabm an Drect an. Slber jag', Eilli, was hat benn mei Doftor, der Dr. șatichel, dazua g'iagt?

(Eilli. Der war erit ganz paff, bann bat er g'jagt: Das g'bört net hierber und bie Frau 3eugin ift jeşt verbeiratet! Stell bir vor, Ferol, idjreit ber jübijche Doftor bazwifchen. Um fo idjlechter, wenn fie ver= beirat is.

Fredinger (Das Rachen verbeiz̄end und Den Bortigen jpielend). Mas? Das hat er g'jagt! It fo a Frechyeit. ?a, wann $i$ den berwifch!!! . . . wann i den berwifh! denn mir

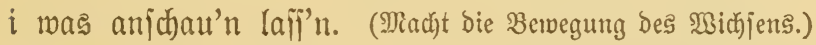

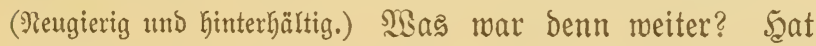
er vielleid)t no was g'idjimpit? Den laif' i auf Ehren= beleibigung llagen.

Cilli (rebet fith immer in gröbere Erregung Ginein). Plet

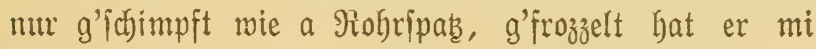
a no!

Freditger. M̉iajo? 
(5illi. M̉ie der Şudenbua g'ichrien hat: $u m$ io ichlechter, wann fie verheirat' is und in glïctlicher she lebt, hat er mi als a lachender g'fragt: Jekt möcht ich nur roijien, wer eigentlich ber (s)lüctliche in biejer Ehe ift. Sie, ba hat er auf mi beut', (zeigt mit bem 3eigejinger auf jich jelbjit) Şhr Mann, ober ber Scerr Fredinger?

Frebintger (auffpringent, mit verítellter Ëmpörung). Eॄ̧ ijt wirffich net zum glauben, was fi heunt io a baber= gelaufener Bamichabl von an jübijchen Irovofaten alles berlaubt. $2 a \tilde{\beta}$ geh'n, (Eilli, (tröitens) $i$ ber şerr von Fredinger, i wir's ifm icho zagn und mei Doftor, der Şatichef, fommt mit'n großem Edymert. (Stellt jich in

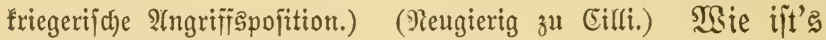
benn aus'gangen?

(billi (berufigter). Şin und her g'ïtritten haben's,

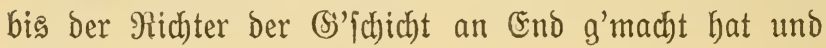
bann -

Jrediuger (unterbrechend, lauerno). Dann haft fhmören müijen.

(Silli. Ş̄t mir eh idjwer anfommen.

Jrediuger (baria)). Das hajt mir ichon amal g'iagt.

(Silli (zögerno, furchtfam). Sa, aber wann's d'rauf fommen, ba za net wahr is.

Fredinger (legt ihr raja) bie Szand auf den Mund, Leife). \$it? Sei itad und red net io an Unjinn? SBas is Denn net wahr? Wie kann's benn net wahr jein?

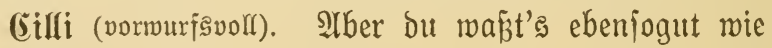

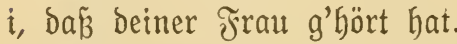

Frediuger. WSirit ruhig fein. Reo net io g'ichmoli'n. 
3'erit jejimpfit und idbreift, $i$ joll mir do mei Eigentum net nehman laijen und es net meinen Rindern, Dera Strabanzabanda laijen und weil's jeķt a bifijel hantig wirb, ziagit ben Schmaf ein und reb'it io an Stiefel

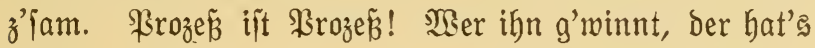
Fecht. Und wia ifn aner g'winnt, das is bem iei eigene Sach' und i g'winn ben \$rrozés, denn $i$ hab's Recht, weil bas Şaus mir g'hört hat, mei ganz allani. Alllss, alles hat nur mir g'hört, (bijfig) oder willï ou,

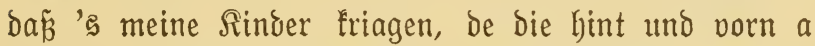
suaber hit und a suaber her g'hajen haben?

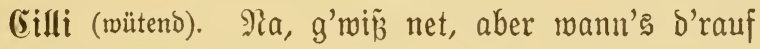
fommen und mi einipirr'n!

Frediuger. Saperlapapp! Braudjit fa 2lngît z'bam. Red fan Stiefel z'lam. $\mathfrak{L} a \tilde{B}$ mur mi und mein Doftor machen. Mei Doftor, ber veriteht's ja, bas is ja net Der erite 'Frozer, Den $i$ g'winn.

(5illi. SMann's bir mur net amal ichlecht ausgeht!

Fredinger. Mas fchlecht? Red net jo oumm, Das veritebit bu net, cilli!

(Silli. Alber mei Mann jagt's auch.

Fredinger (erjobrocten auffahrend). Selijas! Cilli, du wirjt do Deituem Mann nichts davon erzählt hab'n?

(Eilli. Slber gar feine Soee, für gar fo oumm brauchjit mi net z'halten. Şch mein ja mur, er jagt, baź bie Reut icho umananderreben, bei bir miro's wegen beine fortmährenden (Sejdidjten no a idjlechts End nebma.

Fredittger (zornig). Die Reut, bie fönnen mi Buctele fraren tragen uno Dei Mlann, ber foll fie liaber mebr 
um fei Balbiererei fümmern und meniger faufen, bamit $i$ net immer jeine Schulden in bie Mirtghäujer uno Saffeeczecherln zahl'n mua

(Gilli (entrüjtet). 2lber, Ferol, de zablft ja bu gar net! Fredinger. M̧er Denn jonjt?

(billi. Na ich!

Fredinger (höhnifă). Siber mit mem fein Şeld benn? Bielleidft gar mit dein $\mathfrak{B e r b i e n j t ? ~}$

(Cilli (rot und verlegen). Sa, mit mei'm Berbienjt.

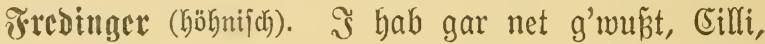

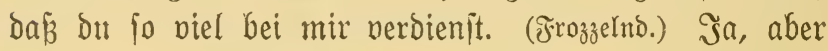
fag mir mur, Cilli, megen mas fefierit bu mi benn immer fo um (Seld, Damit'it die Schulden von bein Mann zahl'n fannit? San bas andere Schulden oder is das a anderer Mann?

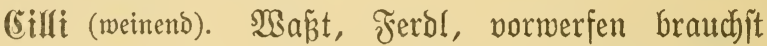
mir bas net? Sch hab mein Mann gern g'babt.

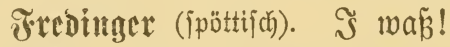

(6illi. Denn $i$ hob ihn aus Riab g'yeirat.

Fredinger. Stber $i$ hab bafür zahl'n müa $\tilde{z}^{\prime} n$.

Cillli. Das war für bie fünf Sinder, die $i$ von bir Kab. Mei Mann hat nidbts bavon g'habt.

Frebinger. Iflles was wahr ijt! (5)’habt hat er nichts davon.

(Silli (befriesigt). গa alfo, jeb̧t gibjt es felber zua.

Fredinger. Sa, weil er's verfoffen hat.

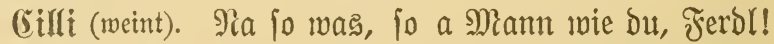

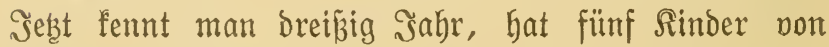
eal)m und er tut an jo was an und wirft an bie paar Gsulden vor, die man für'n Manı braudft, den man 
g'heirat hat. Intio ba tuat ma alles, alles, was man mur tuan fann. Sogar g'iøwwor'n hab $i$.

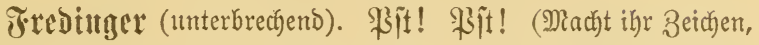

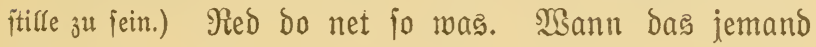
Gör'n tät.

(Gifli (meint). Sa, weils wahr is! Siet amal den Mann gunnit mir, hätt $i$ benn ervig lebig bleiben foll'n?! Jünf Sinder lyab ich ef pon bir alier lediger g'habt uno beim lechjten war $i$ menigitens icho verbeirat.

Fredinger. Stber id hab bir's immer g'jagt, ou follit net heiraten, aber bu warit ja fo vernarrt in ben Mann und hait burchaus Frau werden woll'n.

(Billi (bijifig). Şättejt bu mich vielleicht g'beirat?

Jrebinger. Ra, wer waj̄, wann $i$ lebig g'wejen wär?

(Cilli. Sa, bas jagit jeşt, weil $i$ verheirat bin.

Frediuger (jie unterbreç̧end). Und wannjt net g'Geirat Gätt'it und g'wart Gätt'it, fiefjit, Cilli, jebt bin i Maittiber, aber fo natürli, nadbom bu eh an Mann hait, geht's niminer.

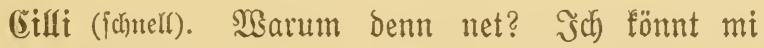
ja jubetben lajfen?!

Jrediuger (eritaunt). Edheiden lafien? Ja. (überlegt und fagt linterlifitig.) Cilli, bas ijt mabr, aber fo recht es mir wär, würo's trobe ber Scheibung nichts aus ber Seirat. (5eucterifor.) Iut mir wirflich recht lad?

(Cifli (enttäujळt). Marum benn net?

Jredinger. M̂Eet mir Ratfolifen jan. 
(5illi. MBiejo Ratholifen! derfen denn be net a zweit's mal heiraten, wann's g'ichieden jan?

Fredinger. So viel $\mathfrak{i}$ war net. Şern hab'n tönnen fie fich a paar mal, aber heiraten nur amal.

(Cilli. Alber bie Rutherifhen, be berfen do wieder heiraten, mann's g'idjieden worden ian?

Fredinger. J゙h glaub' ja.

(Silli (enttäufcht). M̉ann ich bas frïher g'wußst hätt', mar ich a sutherifhe wor'n, bevor i meinen Mann g'heirat hab.

Fredinger. Recht hätt'it g'habt. Sa, ja, i jag's immer; g'icheio jein is ichön, aber wifĩen mua man's beizeiten.

(5illi. 2lber mann $i$ jeşt lutherifd) wurbat, bann funnt i mi aljer lutberiicher icheiden lafjen und bi heiraten.

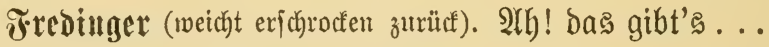
(Unterbricht fich uno bejinnt ficth, vorfictitig.) 's wär mögli. I waß̉ dös net g'nau. Stber wamnit es präzife wifijen millit, fo fannit ja den Dr. Scatichef fragen.:

(Gillt (von ber şbee hingeriffent). Sa, Ferol, ja, bas

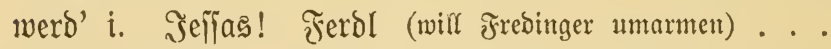
wann idh bich no amal zum Mann friegat. So a (S)lücf bat aber unjeraner net, wann er fich's autch red= (ich) verdient hat, io wie ich. Yllles tät $i$, alles jagat $i$ aus. M̉ann's millit, $i$ geh' no mal zum (s'ridyt uno (c)jö̈r no was bazu!

Fredinger (ifr abuinfent). Suber fei do g'icheio uno

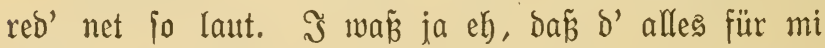
tuajt. Sieb'it, Cilli, $i$ bab' eb glei immer an bi bent, 
i hab' bir bie fietten von meiner Frau ihrem Schmulf ichenfer wollen, aber Der Lausbua, Der Raimund, Der hat mi g'fört und hat an Siramall g'ichlag'n. Da jan bann meine ganzen finder fommen und hab'n mit mir g'ïtritten uno mi abjolut nimmer auslafien, bis i eahna

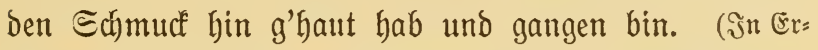

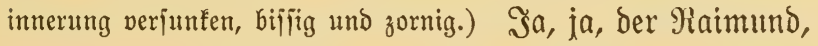

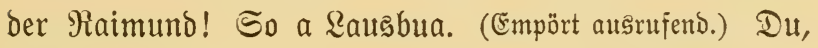
Cilli, aufg'rieben hat er auf mi!

(Silli (entiest). 2łufg'rieben?!

Fredinger. Sa, aufg'rieben. Stell bir por, auf mi, auf jein 3atern hat er aufg'rieben! Irbihau'n hat er nir ane woll'n.

(Silli. Es i ît net zum glauben, was der fich fidjon alles erlaubt. Shabt's denn g'raft miteinander?

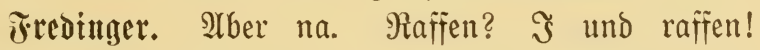
So a friedlicher Mienich wia $i$ und raffen und no bazua mit mein jüngiten Buab'n. Fallert mir ein. s)tber a berwogener fierl ijt er.

(Eilli. Sisie leis's benn bann io J'iammg'raten?

Fredinger. SBegen nir und mieder nix. Er hat mid) g'ieg'n, wia $i$ ben Schmude ang'ichaut hab uns $i$ hab ifm g'jagt, er joll mir ben Ed)mucf nur orbentlid) anichau'n Inifen, ba is er glei rabiat wor'n, hat glei zum ichrei'n ang'fangt, $i$ foll feiner Miutter ifren Sdhmudf fteh'n laijen, Der g'bört net mei und i will ifn eh nur nebmen, bamit idh'n (Euch idhenfen fann. $\mathfrak{Z}\left(\mathfrak{s}_{\text {s }}\right.$ ob $\mathrm{i}$ mi je an frembem Eigentum vergreifen tät! $\Im$ bin a efrlicher Menich und a Charafter und tua io was net! Irber fo hat's eh mei g'hört, denn es is. 
pon mei (Sield fauft mor'n, alio g'fört's mei! und was war benn ba weiter babei, wann i bir a Stïtfl bavon g'ichenft hätt; notabene von mein Esigentum, aber ba

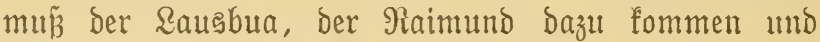
glei fo rabiat wer'n.

(billi. Jch) hab's ja ichon lang g'jagt, baj er a rabiater Ferl is. Schon vor ein paar Эahren. Fiannit di erinnern, wie er no a Bua von a elf, zwölf Salyrl'n war und twie er zu mir fommen is und g'jagt luat: (hocboutich nactafhmend) Son heut an fagen Sie mir Sie

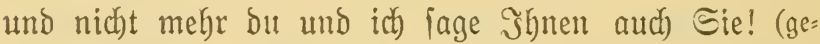
wöhnlich weiteriprect)ens) und wie ich ifhn g'rragt hab, warum ich bin bo mit alle beine (Ssejchwijter no per bu, bat er mir ganz einfach zur ântwort geb'n: (hockdeutich) Megen meiner Mutter!

Jrebinger (nactbenflich). Ja, ja, weg'n jeiner Mutter. Eei Mluatta, an bera is er wirfli g'bängt. Er hat alles tan, was ifym g'jagt hat, bas muás man ifym laijen. Ret fo wia jeine (S)'ichwijter, be a vis=a=pis eahnara Mutter a verlogene, Ginterlifitige Banda war'n. Der Maimuno, ber wär für fei Mlutter Durchs Fetter gang'n.

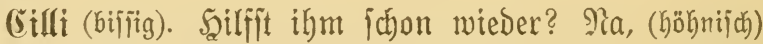
ou vergípt ichon mieba, daßj er net von...

Fredinger (mütend, grob auffahrend). Sei ftab. Salt's

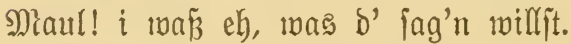

(Eilli (ipitigig). Du hajt's ja jelber g'jagt.

Fredinger (grob). Da hab i's halt g'jagt. I mag'n eh net.

(silli. Ş马 eh am bejten, denn $i$ wá̉ wirkli net, 
was alle an bem Buabn g'frefīen hab'u. Sect is er, jaugrob is er, und z'iammg'ichimpt hat er uns alle.

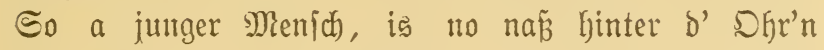
und wann ma mit jemandem von ifm redt, gibt ei'm

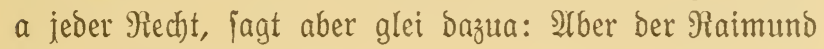
is a anitändiger Menich.

\section{Frediutger (befräftigeno). Das is er $a$.}

(billi (hojmijh). Ia hamers ja. Seest jagit es jelber, bu, ber ifn net mag und bem er ane abihau'n lyat woll'n. (Boshaft uno höhnifa aufladjens). Der Piaimunto a anjtändiger Menic)! Marum jagt Denn bas a jeber? Warum benn? Meil er vielleidjt gegen unjeraner grob und gemein is? Doer meil er was g'lernt hat uno ins Univerfium geft. $\mathfrak{I}$ bin zivar net its Univerfium gang'n umb hab net viel g'lernt, Denn zu meiner 3eit hat man fïtr folche Dummtheiten fa Beit g'habt, aber bin i beshalb net was mor'n? Mir fann ficher do niemand mas anders nachjag'n, als baj̄ $i$ immer an= ftändig war und mir mei Brot ehrlich perbient hab. (5)'raubt uno g'itohl'n hab i net.

\section{Fredinger (ironif(a)). Net?}

(Eilli. Und mann idh bas net tan hab, bin $i$ do ficher ebenjo anitänoig wie ber Faimund und Der fagt,

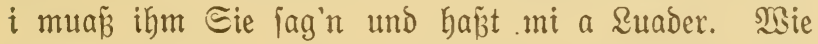
fann man benn a brav's Madl und an achtbare Frau fo fchimpfen? SGeil ich dir jeit meinem 15. Sahr ehr= lich und redlich bient hab, oder weil $i$ a paar finder pon Dir hab? Sit benn ba was babei? Jich hab's halt aljer lediger auf ঠ' Melt bracht und fei Muatter alfer verheirateter und da fagt er, meine Sinder jan Bajtardin. 
$2(15$ ob bas net g'logen wie brudft wär? Bajtarbln fan boch unr Rinder von ledige Reut, bas ijt aber net bei uns ber Fall, benn wann $i$ a felber no lebig war, wie ich bie Sinder von bir friagt hab, fo marit ja bu ichon verheirat, alitern jan's fane Bajtaroln. Sa, hob $i$ net Recht, Ferol?

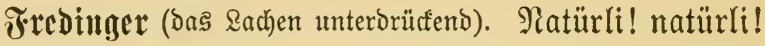

(billi (immer eifriger merbent). Und wie war's benn g'wejen, wann's bu mi früher fennt hätt'ît wia dei Frau, do waret bann fie bas suader und ber Raimund a Bajtarbl!

Frebinger (auf ben Iif und bu warft mei Frau woro'n uno dei groap̃er $\mathfrak{B u a}$ hätt fitubiert.

(Silli (freudig). Mas, Ferol, wie guat fie bas g'mad)t bätt. Und er war a net burd)g'fallen, benn mei Bub, ber Schani, hat jo viel an offenen Sopf. Da lagen d' Reut, er hat an Maiferfopf. Das ijt ja gar net wahr! als ob mei $\mathfrak{B u b}$ an $\mathfrak{M a f f e r f o p f}$ haben funnt! 's îj ja gar net möglich, denn wie er vierzehn Sahr

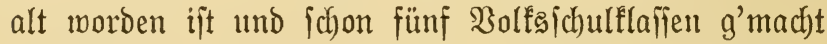
g'habt hat, ba hat's a g'baß̉en, mei B̧ua, ber Schani, war in jeder Slaff' fiben blieb'n, aber gar fa (s'ipur ba bavon, ich, fei Muatter, wir's do am beften wiffen. Der $\mathfrak{B u b}$ macht alles recht gründi, Damit er's guat wấ und bes!negen hat er a a jede filafi' zwamol g'macht. Und ba fag'n b' Reut, er is fitzen blieben.

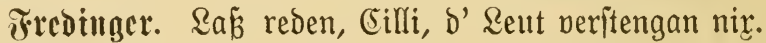

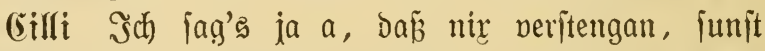
fuunten's net fo was j'iammreben, denn mir bat's 
mei'm B̧uab'n lei Refrer ielber g'iagt, wie mei Schani vierzegn Jahr alt wor'n is. Da hab id) mit jei'm Refrer felber g'redt und hab ifhm g'iagt, i möcht den Buab'n meiter itubieren laijen, wann's mir a no fo id)wer anfäm. Denn mei Schani hat fo viel an offenten Siopf und lernt fo leidst und hat aus lauter Siab zum Sernen jebe Silaif' zweimal wiederfolt und ba hab i halt Den Rehrer g'fragt, was er bazı meinert, ob ich ihn net jeb̧t in a höhere ङchul' ichicfen fönnt. Der lyat mi

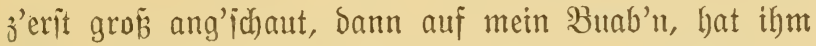
feine Sand auf Den Siopf g'legt und aljer lachender zu mir g'fagt: Sic haben Yiecht, metne lieke Jrau, Shr Sohn hat, wie idh mich überzeugt habe, wirflid) einen offenen Siopf und einen zu offenen Sopf für meitere Studien; es wäre wirflidf lüade, menn der bem 5̧and= wert perloren ginge.

Jrredinger (biifiig). Sat er bas mirffich g'iagt?

(silli. Ssantj natïrli hat er's g'iagt. Şch bab' bir's feinerzeit eh derzählt, aber ou fannit bi wahricheinlich

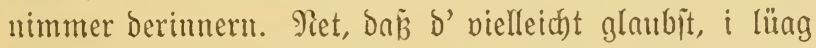
Dir was vor, aber iei Rehrer hat'n wirfli io g'lobt, na und da is er halt bas word'n, was jei Sater is, bas

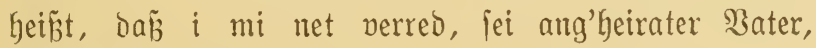
nämlich Balbierer.

Fredinger. 5̧ait a ganz recht g'tabt, benn bie Balbiererei is a fitnit, net io wia be blöbe Stubiererei.

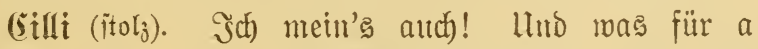
Siunit! Tas, was ber Maimund fann, lernt mei Schanerl im Sandumoref'n. SBas itt Denn a (S)umnafium, 
Do wer'n die Bub'n zu Trotteln ergog'n und im 4 ni= verfium, ba wern's nix nut.

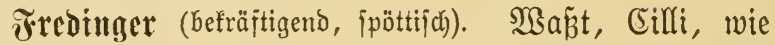
bu alles swä̈t und ben Sagel auf'n Sopf triffit. Es ijt wirflich mahr, im Univerfium mer' $n$ ' jungen $\mathfrak{L}_{\text {eut }}$ wirfli nix mus, bös haß̉t wann's hingeh'n, aber viel

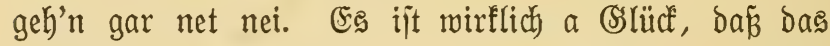
bein Schanerl net g'idjef'n is, benn als Balbiererg'bilf' is er do ganz wer anderer.

(Cîlli (nit jtolzer ßetomung). (Sallz wer anderer?! Wie ich ichon g'jagt hab, mann mei $\mathfrak{b u b}$ mecht, lernat er im Scandumbreken das, was ber Maimund fann, aber ber Saimund foll's probieren, ob er an o' Şaar ichneiden oder balbieren fann.

Fredinger. 2lber ber! 2in Galbieren finna oder gar d' Scaar ichneiden! Dá̉ i net lach! Das hat er ja net g'itudiert, ba is er z' bumm bazu!

(Cilli (freubig). Ret mahr? Jch lag's ja immer, es is nur a bumme Peonerei von d' Reut, wann's jag'n,

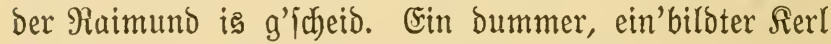
is er, ber fi fei Brot net felber verbienen lann und Dabei gegen fo anjtändige, vernünftige Reut, wie unier= an's is, auftritt, als wär ma wirfli a Straßßenmentid und net an adhtbare Frau. Hnd wie red't er benn mit bir, Ferol? Scat er an 2ld dutung vor bir?

Fredinger (ichüttelt unjalüifitg ben Ropi).

(Cilli. 2Yuf fein eig'nen Satern reibt er auf, ber's ifm immer fo guat g'meint hat.

Frediuger (heuchlerija)). Ind wia guat id, ifm's 
immer g'mant lab. Irfles hab id ifm zufommen laijen. 2rlles, megr wie jedem anderen.

(Silli. Da Derzähl'n fich aber d' Lent, da fei Muatter tot is, er von bir net amal g'mug z' efien

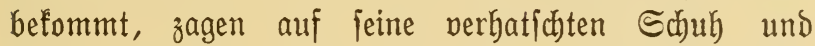
g'flicften Flaber und jag'n ganz laut, bá er net amal 's notwendigite friegt.

Fredinger. Was, Eilli? Mir bas! Mir, dem 5eern von Fredinger! Der $i$ immer fo a guter $\mathfrak{B}$ ater für meine Rinder mar! Nber be Strabanzerbantoa fann's net fbäben und ber Rausbua, ber Raimund am allermenigiten. $\Im$ hab überhaupt a (slüct mit meiner Familie. Mei Frau be... (unterbricht fich) na, i will liaber nix reben, fie is jebst in Der (Siruab'n, (Geudjerija) ınier Seerrgott laif' ruf'n, aber was is mit be finder. Der ßepi is a leidt's $\mathfrak{I} u d$, der alles verlumpt und verbrabt. (Bemugtueno.) Sia, jebt fann er mir nir melyr nehmen, benn wie er mit ieine (S'jichmijter z'iammgang'n is in bem \$rozez, hab i ifn auffitg'ichmifif'n. Stell bir vor, Cilli, de bab'n mi burch eahnern Doftor mi fequejtrieren laij'n woll'n. গa, woart nur, bu rot= g'ichäbleder Rump, na, wart mur (mit broheno ausgejtertecter そauft).

(Eilli. Marum lañt's du dir's denn g'fallen?

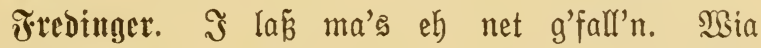
g'jagt, ber \$epi, das is a sump, ber oraht nno fauft, bie Madln, de fan Siarnallina, wia ma's net io balo wieber find't, und mei Sdyiegerfuhn, ber Lampeldiab, ber hat mir g'rad no g'feflt. Sa, ja, aber's Iejtament

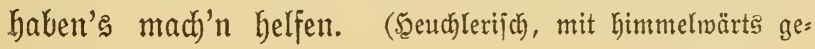




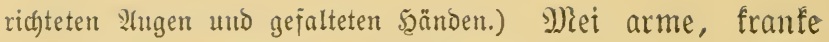
Jrau! io z' martern! hätten i's net ruhig iterben laij'n

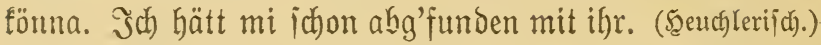
Denn $i$ bin joviel an elyrlicher uno anjtänoiger Menich uno a Eharafter. $\Im$ hab mei Frau nia leftert, ite foll mir ifre Sachen vermachen. $\Im$ tua jo mas net, aber meine finder, be haben's beijer veritanden, be haben woll'n, i joll gar nix friag'n. Tiet amal ielber hab'n iie ïch's vergunnt, demn bie $\mathfrak{B} u b$ 'n fan jo z' furz

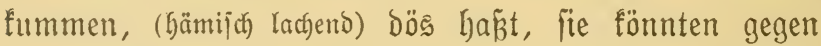
eahnere હchmeitern z' furz fommen, wann's b' (Erbichaft mirflich frieg'n tuan, und dem Maimund ham's net amal bas biifel Bargeld vergumnt, was alle friagt ham, bös haß̄t frieg'n joll'n, (Göhnija) aber bis dahin, da hat's no 3eit. Pa ja, bie zma Mabeln, mei Schwieger= jun, ber Rampeldiab, bie hab'n ja 's Iejtament g'macht, da mar ja net mir für mi, fonbern a für eahnera Brïber net mehr zu ermarten!

(5illi (einjallemb). Stber dá̉ nur det テrrau dazua brad)t bam?

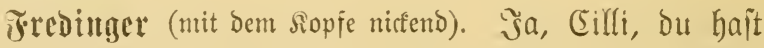
Hecht, da hab i mir idfon oft meine Bsedanfen brïber g'madtst, wie fie's ang'îtellt ham, dañ mei frau a folches Iejtament g'macht hat. Ia hätt i $u$ m alles g'wett', Dá̉ bie Bub'n, be do ifre Riebling waren, menigitens io viel als d' Madeln frieg'n, aber ba $\tilde{B}$ de weniger frieg'n als wie D' Madln und bá̉ Der Maimuno, Der

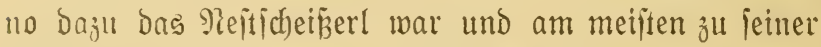
Mutter g'balten hat, an allerwenigĩten friegt, bätt $i$ um alles in ber siselt net glaubt. Fa, bei ser 
Teftamentseröfinung hat eh Der Maamumo g'idjrie'n: "Das iit Raub" und is fortg'rennt und bie Mizi und die Toni fan ifm nachg'rennt und hab'n ifm zureden troll'n. Sistro ealyner halt 's (S'mifĩen oructit bab'n und fe hab'n an 2Ingit g'tabt, es fummt was auf, (ben Sopf fojüttelnd) Denn fo ganz ofyne ijt's bei bem Ieftament net zuagangen. Mei Frau und io a Ieita= ment? Ess is mir heunt no a Rätiel, wia bas hat g'ichef'n fönnen.

(silli. Mi hat's a g'wunbert.

Fredinger. $\Im$ hab el glaubt, $i$ wir be Teftament= macherei überhaupt verhindern fönnen, aber es is nimmer gang'n. Die ßeterlin lyat mi zroar glei g'tyolt

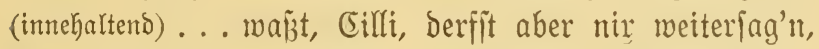
bie Peterlin hat mir alles zutrag'n, was in ber $\mathfrak{M B}_{\mathrm{b}} \mathrm{h}=$

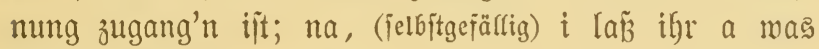
bafïr zufommen.

Cilli. Lnd bamn 'mur je's a tuan!

Fredinger (Den Eriftaunten ipielend). M̉iefo?

Cuilli. Du hajt's mir's ja jelber g'iagt, da g'îtohl'n hat.

Frediutuer (Ginterlititig). S foll bas g'jagt hab'n?!

(silli (beleibigt). S wir bir do net fo ins (s')ficht

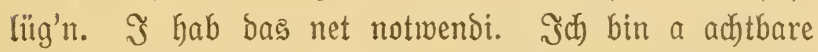
Frau. Es ijt aber net fo lang, baí d' mir erzählt hait, dáp bie Sieterlin von beiner Fraut ifre Sachen joviel

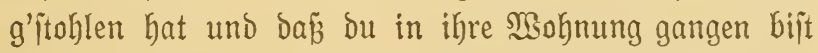
und bie Mohnung ganz an'gramt g'funden hajt mit 
lauter Sad)en, De's beiner veritorbenen Frau g'itohlen hat. (s)laubit benn, ich) werb mir jorwas aus dem finger ¡uzzeln?

Frediuger. SMannit du's fdyon waßst, fo fanu $i$ net mebr ua jag'n. 2(ber du barfit nichts weiterreden,

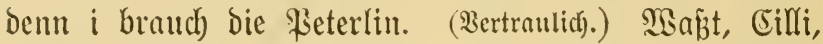

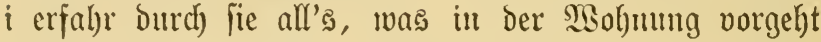
(ichlau) und no melyr. Drum braud) $i$ die \$seterlin. Sie hat g'itohl'n und is a groỉ suaber, aber a santo wajkt bie andere und bei bell Erbichafteprozes, ben $i$ jekst gegen meine Sinder fülyr, muaj i's hab'n.

(billi (eritaunt). Soll be vielleicht a ausfag'n? De fam bir ja met belfen.

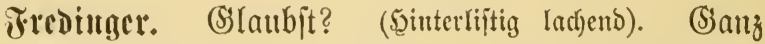
matïrli! Soll autsag'n?! Siet mur foll, fie hat icho aแsg'iagt und fo, wia i's berwart hab, mur bie reine

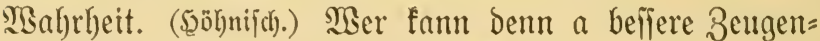
ichaft abgeben, als wir a \$erion, auf be mei veritorbente Frau fo viel g'balten hat?! (Sid) itor Siannit ganz itab fein, Cilli, i waj mir meine Rent (d)on altsj’łud)en.

(હ゙ふ flopft.)

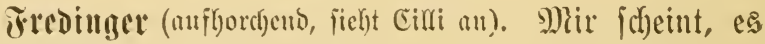
hat flopft.

(oilli. M)ir idjeint a!

istedinger (rant). Deerein! 


\section{Pldite Szente.}

Die Borigen. Dr. Saatidet.

Dr. Sृatia)ef (öfïnt bie mittlere Türe, fieht vorerit herein, und als er Fredinger fieht, geht er mit ausgeftrecten Sänden auf ihn zu). (Srü̈ß Sie SSott, Seerr pon Fredinger!

Fredinger (als er bemerft, baj es Dr. Satjchef ijt, auf= fpringend und entgegeneilend, jehr freundich, beinabe bevot).

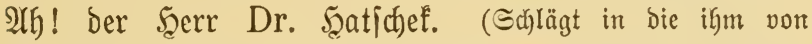
Dr. Şatjchef entgegengereichten Şände ein.) Şabe die Shre, Seerr Doftor!

Dr. Şatiduct. Wie geljt's, Jeerr pon Fredinger?

Fredinger. Danfe fïr o' Rachfrag! Šmmer gleidh, immer gleich, Şerr Doftor, und Э̧hen, Şerr Doftor?

Dr. Szatidjef. Dante, Den Umitänden angemelien. Tredinger (fieht ihn erwartungsvoll an und gibt ifm einen Binf betreffs Cilli). Mুas gibt's neues, Scerr Doftor?

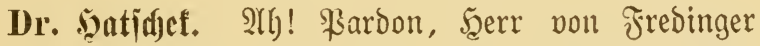

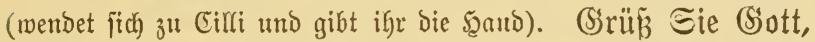
Frau Eilli!

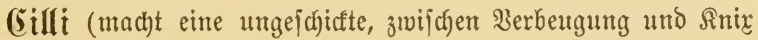
[chtwanfende Bemegung). Эch hab bie Ehre, Scerr Doftor!

Dr. Şatidjef (neigt fid zu ihr). Şaben Sie Şerrn von Fredinger fchon Mitteilung gemacht?

(5illi. Ja, Seerr Doftor, g'rad jeşt.

Fredinger (einfallend). 2rlles hat's ma berzählt, alles, Seerr Doftor.

Dr. Şatjdet. Dann ift's ja gut. AUljo leben's

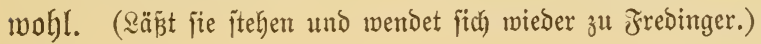


Fredinger (ba Cifli nidgt verjtehen will uno nicht geht,

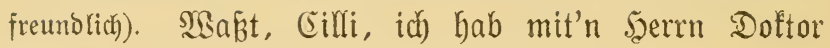
was z' reben, i la

(Cilli (jiefht erịt eriftaunt unb beleibigt auf und will etwas er:

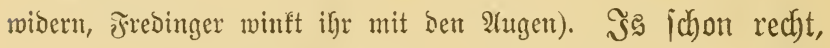
mann's mi brauch'n, laffen's mi wieder ruafen, seerr pon Freoinger.

Fredinger. (Sstrat $i s$.

(Silli (macht wieber ifre ungeichicfte, 3̧wijd)en Berbeugung und

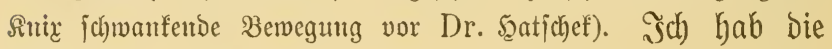
E⿹hre, heerr Doftor.

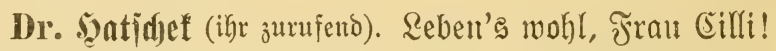
(Sronifo.) Sffitat Shna (Sott!

(Eilli ab.)

\section{Teunte Szene. \\ Frebinger und Dr. 5atfichef.}

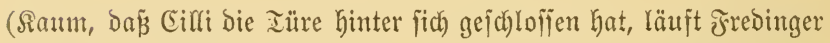
hjin und verriegelt biejelbe fowohl als auch bie andere Türe.)

Fredinger. SYln Moment, 5eerr Doftor. (2äujt zur Türe.)

Dr. Şatidjef. Bitte, bitte, Şerr von Frebinger!

Fredinger (zurücfelfrent). Es is nur, bamit niemand einafummt und roir ung'itört jan. (Beht zu feinem Schreib= tifa und bietet Dr. Fantichet mit einer Şandbemegung einen Stuhl an ber Seite besferben an, fid felber jeţento.) Bitte, feben's Şhna, Sgerr Doftor, wir fennen do net ewig ittehu.

Dr. Satidjef (jeșt jith). Danf fchön, Seerr von Frebinger. 


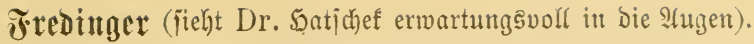
Ulitern, was gibt's benn nettes, 5eer Doftor?

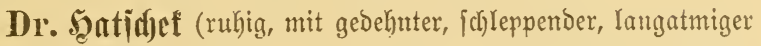
Stinme). 2rljo, wie ich börte, hat Şhnen, ફ̧err von Fredinger, die Frau Eilli bereits alles erzählt be= treff̈s ihrer Slusjage?

Jrevinger (Yächelño). $\Im a !$

Dr. satidjef (idmmuzelno). Es ijt jedenfalls ein einigermaß̉en unangenebmer und peinlicher 3mijchenfall gemejen, wirb aber am (Sange bes Frozelies nicht bas geringite ändern. Die 2lusjage an uno für fïch murbe als vollwertig anerfannt. Im grofenen und ganzen itebt bie Âtngelegenheit günitig. Sie jeb̧t hat fein von uns geführter 3enge ausgelaijen. (5s iît wirflich) ein präch)= tiges Beugenmaterial.

Frediuger (voriftrtig, reife). Saben's alle uniere Beugen a ichrör'n laijen, 5̧err Doftor?

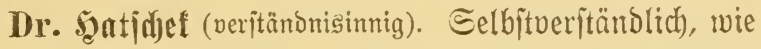
wir es zu Beginn bes Srozelies bejprochen. Mir merben uns boch nicht einer eventuellen 3urüdfnabme

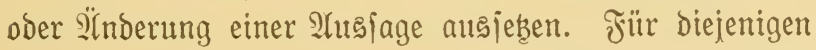
Beugen, weldbe ifre 2lusfage ichon abgelegt haben, gibt es fein Burüct, (betonend) fein Burïd mehr. Sie haben alle ifre Ilugfagen beeibet uno bei jold)en, wo man vielleidyt trob alledem noch eine nachträglicje Sinnes: änderung ermarten fönnte, werben wir, wie o. $\mathfrak{B}$. bei

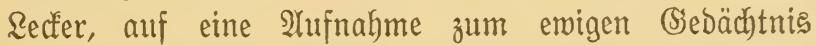
bringen. In Invetracht Der Ronjequenzen, rvelche für bie Beugen entittehen fönnten, gibt es für fie nunmebr fein Burücf́. 
Fredinger (befriesigt). $\Im$ hab nur g'mant, bie Menichen fan ichlecht, und wann's an was antun fönna.

Dr. Scatidjef (betonend). So mürben fie fïd nur um fo tiefer ins eigene Fleiīd ichneiden. (Selbitgefällig.) $D a$ fönnen Sie ganz beruhigt fein. Umionit ift man nicht 2ldoofat und veriteft feinen Beruf.

Fredinger (nift mit bem Sopfe). Das twa $\mathfrak{i}$ mur $\mathfrak{z} u$ gut, Dás Sö's guat verftängan.

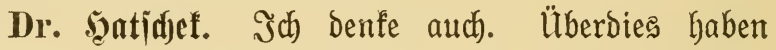
uns bie von mir bem (Serichte als Berweigdofumente

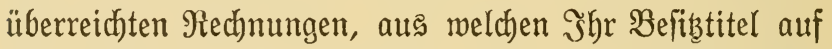
Das itrittige Dbjeft hernorgeht, utm ein ichönes Stür in ber Rette ber Beweife vormärts gebracht.

Frediuger. Uno jeşt, g'rad vorher, hab i Sgna

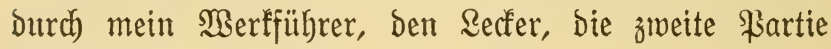
einig'idjičtt.

Dr. Şatidist. Sa, ich habe biefelben, fluapp bevor ich) zu Shnen ging, noch übernommen uno werbe fie fo= fort Dem (Serichte einantworten.

Frredinger (iłn ungedurdig unterbrectgent). Sagt er Şhnen Derzäblt, Seerr Doftor?

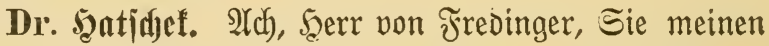
wegen bem Fonigl. Sa, bies war audi ein (Srumb, rwestwegen idh) gleidh) zu Şhnen gefommen. NTber ba braudjen Sie fict) vorberband fein graues Şaar wadbjen laifien.

Fredinger (funthn und frect). NYGer was fagen's Denn zu io aner Freeffyeit? Mir, bem Seerrn von Frebinger, Der ich fo a ebrlicher Menfich bin und a EGarafter! 
Dr. Santidjef. Mian fann von foldben Reuten nidats anderes erwarten. Piegen Sie fich bafher besmegen nicht auf. Menn es not tut, werben wir mit bem frechen Burjichen fibon fertig werben und werbe auch in biejer ßidbtung Borjorge treffen. Sm übrigen, Seerr vou Frebinger, fönnen wir, wie bie Sache iteht, ganz zu= frieben fein und bin ich auch biejethalb gefommen, um mit Эhnen eine nähere ßepprechung zu pflegen. Mie gejagt, Scerr von Frebinger, nach dem jebigen Stand

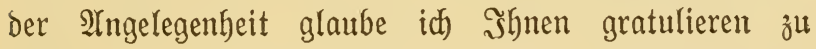
fönnen, denn, wie idh Shnen bereits vor Beginn bes Frozelfes und jeitfer wieberholt jagte, zu geminnen ift Der ßrozez nie, da bie grundbücherliche Eintragung des Scaujes auf Den Mamen Shrer veriftorbenen Frau, forwie Der gemeinjame Erwerb (శresinger will imterbrechen, Dr. Şatjchef abwehreno), bitte, Scerr von Frebinger, mich ausfpredben zu lafien, jowie ber gemeinjame Errwerb, D. h. Die langjährige Mitarbeit Şhrer verftorbenen Frau, ein nicht abzumeijendes (Segengerwicht gegen unjere $\mathfrak{B e}=$ hauptungen billdet. - Mir müffien baher trachten, falls wir vielleidht bod) nidft (mit erföhter Stimme betoneno) burch) ganz auß̧ergewöhnliche llmptände in Dem \$rozen objïegen, zu einem für uns möglichit günjtigen 2 usgleich zu ge= langen. 3t biejem 3wecfe...

Fredinger (unterbridgt ign ungeduldig). Şaben 5̧err Doftor mit Dem Dr. Seger von meine Sinder g'redt?

Dr. Santidef. Jamofl hab idj bies, aber mein werter gegnerifher Şerr Sollege wird jedesmal teurer, wern id) mit ihm ipreche. (Segenwärtig itellt er fold erorbitante Forderungen, dá̧ 
Fredinger (unterbrectiend). Für fich jelber ober für meine Sinder?

Dr. Şatidjef. Ias exftere ijt ber Fall.

Fredinger. 2ump!

Dr. Şatidat (mit B̉etonung). 2lber natürlich nur für feine Expenjen uno $\mathfrak{A}$ เిlagen.

Frediuger (veritänonišvoll nicfend). $\Im$ veritel), i ver= fiteh! So a Rump!

Dr. Sentidnef. Arber Seerr von Fredinger! Das i ĩt

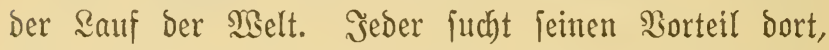
wo er ifn findet. Mie gejagt, gegenmärtig itellt er joldh) enorme SInjpriict)e, dás es mir unmöglich ift, Shnen zu empfehlen, darauf einzugehen.

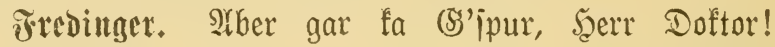
bem wer i's in ben Rachen idhiab'n!

Dr. seatidget. Ulmiomehr, als bod) der Römenanteil uns verbleiben mun.

Fredinger. Ratïrli, zll was führat ma denn bann \$rozeß́!

Dr. Sentidut. Selbitredend! Es bleift uns baber, wie ich es auch Şgnen itets empfohlen, nichts anderes ïbrig, als den (Sang bes \$rozefies nod) mehr zu ver: fajleppen, b. h. zu perlangiamen, als wie wir es bis: her getan.

Frroinger. 2lber warum benn gar fo lang?

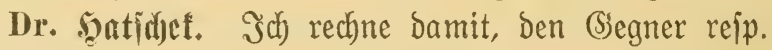
Shre Rinder mürbe zu machen.

Fredinger. शth fo!

Dr. seatidjef. W̉ie Sie mir fagten, find bie Mittel, ïber melche Э̧hre Rinder verfügen, geringfügige? 
Trebiuger. Sa, aber mei Edwiegerjun, ser Schubiaf, der hat a Sseld.

Dr. satidyet. Nun, wird ber für bas allgemeine $\mathfrak{S O}_{\mathrm{b}} \mathrm{l}$ ber anderen auffommen?

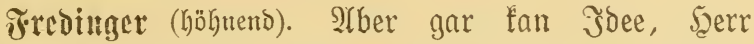
Doftor! Der Echubiaf was hergeb'n! Der nimmt eafner eher's Mart aus bie Snochen.

Dr. Syatidjef. $\mathfrak{H m}$ io befifer, bas iit, was wir für unjere 3wecte brauthen, benn je geringfügiger bie Mittel Shrer Rinder find, bejto eher werden biefelben zu Ende gehen und um io früher werden Эgre Simber zu einem billigen Sltuggleich bereit fein.

Frebinger (eifrig). Ratürli billig! Denn das saus ijt mein Eigentum uno wann i eahna mas gib', is is mur a (Snad' von mir.

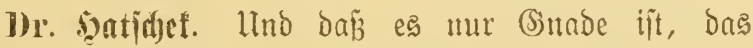
müffen şgre Sinder erit ichäken lemen und barum müiffen wir fie erit bahinbringen. Der bejte $\mathrm{S}_{\mathrm{Seg}}$ hieju, Serr von Fredinger, iit der Э̧nnen bereits angedentete. Ias möglichit weite Sinausfchieben des Frozelies, ba= burch werden Эgre finder bald dem Richts gegenüber fteben und mürbe me:den und bann um fo billiger zu Gaben jein. Althhungern jei bie forarole!

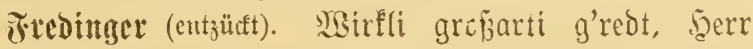
Doftor! 厄ö fan wirfli a g'idjeibter Mann. (Dr. Şat=

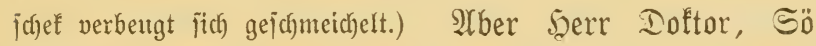
erlauben ichon, was wirb benn ber Dr. Seger bazua jag'n? So viel ma von eafym bis jebt g'mirft hab'n, wird fie ber net io leicht abipeif'n laij'n?

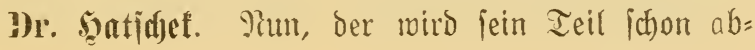


befommen, aber (mit erhöbter Betonung) natïrlid) in be= bedeutent verringertem Miaje, wie er benfelben bean=

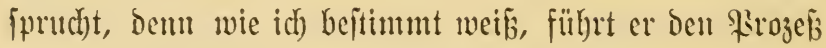
nur in ber Sorausfict)t, baf fïr ihn ielbit cin tïchtiger

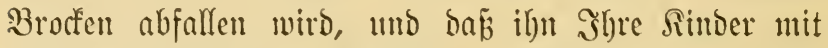

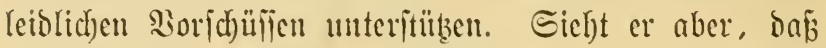
er von Sl)ren Sintorn, von weldben er bisher nidbt cimen roten sefler gejelyen, aud) in şinfunft nid)t et= halten mirb, da fie ja mit Slumahme Shres Schuteger= johnes, refp. feiner Frau, b. h. Shrer vertheirateten Iodjter, jelbit in Rïrze nidyte haben merden, biejelben baburd) mürbe geworden, auf ifren Miedjtsvertreter Dr. Jeger jelbit einen Irud ausïben werden, fo wird mein (mit ironif(cher 3 ctommtg) fehr efrenwerter Soer follege Dr. Jeger, um für fid nod) zu retten, was ju retten ijt, fein beil wieder bei ums fuchen und wenn wir ifm mit einem (mad)t bic bementfprechente 3 cwegung unb betorent) ausgiebigen saändedunct entgegenfommen, Sie wifïen, 5err von Frebinger, eine 5anto wäjdyt bie anbere fo wiro uns Dr. Seger jelbit ju einem möglidbjt vor:

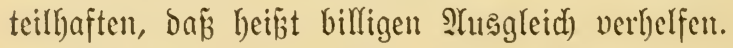

Frediuger (bewumberno). SIrfe Irdetumig vor Şgna, Serr Doftor. Mia Sö bas fdjön jul bringen veritengan.

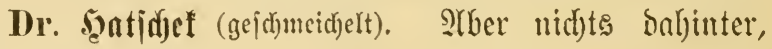
Seert von Frebinger. Randlänfige Sadhe. Sagt bod) fchon unjer gröpter Dichter Slltmeifter (Soethe: Miann mit zugefnüpften $\mathfrak{I} a f d$ en, bir tut niemano mas zu lieb. Şand wiro mur von Şand gewaidjen: SBenn bu nebmen willit, fo gib!

Fredinger. Der hat net llured)t g'babt, bas war 
a g'icheiter Mann. Der muá̃ d' Melt fennt uno ver= ftanden baben.

Dr. Şatideft. Und ob! denn ber Mann war Minifter!

Fredinger. Sia, bann glaub i's, benn jo a Exzellenz= herr mur viel fenna, ber beitelt die (S'jheibheit nur jo aus bem 2̂̈rmel. Das is ja mur natürli, dañ io aner 's no viel beffer fam, wie un'raner.

Dr. şatidjef (zuítimmend lätgelno). Zmeifelsohne. Illo wie gejagt, auf bieje Meife hoffe ich, baj woir zu einem zufriedeniftellenden Ende gelangen merben. Irlpo nod)mals, Şerr von Frebinger, (nit Dem Beigejinger zur Befräftigung feiner Borte brohend) Lafien Sie Shren Sindern gar nichts zulommen.

Frediutger (höhnija). Stber net amal an lucfaten Szeller friag'ns, Şerr Doftor. Meder o' Madin, no

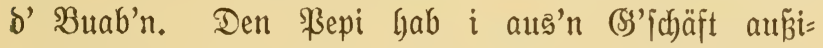

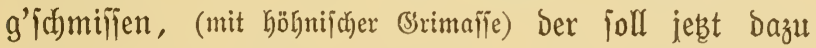
ichau'n, wo a Sseld hernehmen. Denen Madlu gib $i$ a nix mehr, de foll'n meinetwegen auf $\delta$ ' Straßzen geh'n, wann's nix z'elien hab'n; den 5aushalt lös $i$ all, bas bab i meiner Iodjter, ber Ioni, ichon g'iagt, fie foll hingeh'n, wo's will. Bon mir friagt's nix mebr.

Dr. Scatidyet. Sie find bierzu auth nicht ver= pflichtet! Shre Töbter haben, mit einer anjebnlichen Mitgift ausgeftattet, geheiratet und find ïberbies, forwie

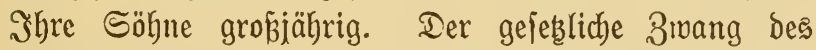
Rebensunterhaltes ift aljo nicht mebr vorbanden uno 
(bejinnt jidf, jubeigt einen Moment und ipricht banu ruhig weiter) aber Şbr jüngiter હohn, Jeerr von Jrebinger?

Jrroinger (jornig). Ee manen den হausbuab'n, den Raimuns? Ien bab i auñig'idymillen.

Dr. Şatidjef. Şinauggemorfen?

Frebinger. Sanz natïrli. S wir bo fame (S'jidjidften mach'n mit Dem ?obbuab'n. Ţo grab norber war er da bei mir, hat mit mir g'itritten, weil $i$ ifm fa (S)eld geb'ı hab.

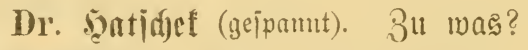

Jrebinger. Iamit er leben fann.

Dr. Jatidjef. jaben ङie ifm etruas gegeben?

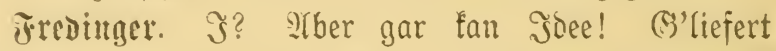
gab i ifn und hab ifym g'jagt, er joll tuan und madf'n was er will. $\mathfrak{s}$ gif ifm nichte.

Dr. Satidef. Ilno bamit hat er ïd zufrieden ge= geben?

Jredinger. S)tuañ fie wohl z'frieden geben. Rann ja nix tuan.

Dr. B̈ntidef. Dod)! Dod)! Serr von Frredinger. sch mache Sie nur barauf aufmerfiam, benn el iit noch nicht grofijährig.

Frediuger (laut uno jrect). (Srrobjährig bin, grob́= jäfrig her, da idher i mi ben blau'n Ieifel D'rum.

D1. Dontid)ef. ìd) madje Eie nux aujmerfiam, 5etr von Frebinger, denn Gie fönnen baju verbalten werden, ibm den Rebensunterbalt ju geben.

Trediuger (irouich). Ilber Serr Doftor, Dös was i belier? Siger foll mi Denn bajua verhalten?

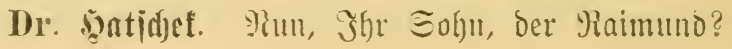




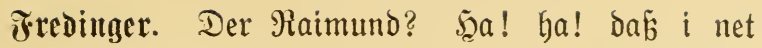

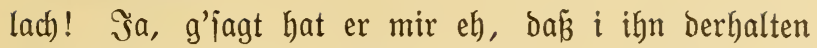

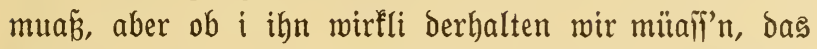
ijt erit a Frag', bie i felber zu beantworten mir' ivifien.

Dr. Satidjef. Tun und menn er Sie flagt? oder wenn Dr. Jeger in feinem Samen gegen Sie tlagbar auftritt?

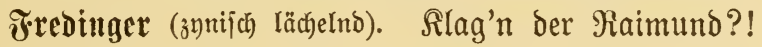
Mi?! Fein Satern?! Mas Shna net ei'fallt, Şerr Doftor, bas berleben's nöt! Marum benn net gar? Der Maimund mi flag'n oder burch fein Doftor flag'n laijen! Der halt viel eher fein Doftor vom flag'n ab.

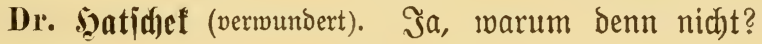
Er märe hierzu vollfommen bered)tigt.

Fredinger. Berechtigt hin, bered)tigt her. Âtber Der Rainutno tuat's nöt.

Dr. Jatidjet (immer vermunderter). Ilber warum benn

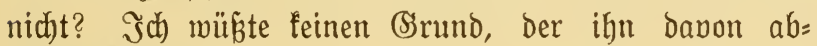
halten fönnte.

Fredinger. Dafür wak i's umio befier, Şerr Doftor.

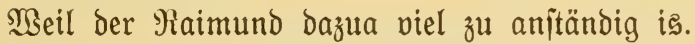

Dr. Santidjet (ïtuşt einen Moment und jagt bann zunifich lädyelnઠ). Zu anjtänoig! Hmio beĩer! Run, da fönnen twir auth in biejer şinfitcht ganz ruhig fein. (Sarfajtifo.) Es hat boch mandidmal auch was (siutes an fitch, wenn ein Menich zu anjtändig ift. -

Frediuger. Fa aljo, Feg'ns Seerr Doftor, dá̉ $i$ Hecht g'babt hab.

Dr. Sentidict. Pau ja, bas itelle ich Sgnen num nicht mehr in wrbrede, aber auf jo roas war ich nicht vorbereitet, denn jelbît in meiner langiäbrigen, viel= 
fältigen Sraries als Nlonofat iit mir bies noch nidyt untergefommen. Ia um eine Rlage erbeben zu fönnen, bas bab ich oft er= lebt, bá aber einer zu anitänoig war, um bies zu tun,

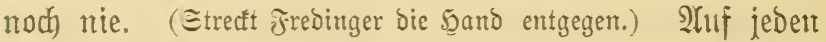
Fall fann ich Şnen nicht mur zu Shren Beugen, iondern auth) ju einem joldfen (Ssegner gratulieren; wenn Эgre anderen finder auci) io wären, würben wir den Frožé leicht gerwinuten.

Jredinger. Sth, be jan net jo bumm, hätt $i$ bei= nah g'iagt, mill jag'n anjtändi. Yrber நeerr Doftor, i

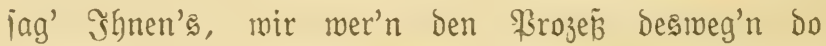
g'rinna, den $i$ bin im Mecht. Mein hat bas şaus

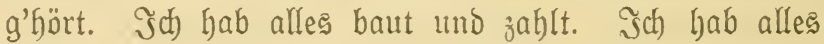
ermorben. (Aufipringend.) Seilig ijt bas Eigentum uno i bin a ebrlicher, rechtichaffener Menich uno a Charafter und mei şaus is an altes, an ehrliches 5zaus. (\$nit beichwörent zum Sinntnel gehobenen Sänden.) Ter alte Biott Da oben, ber lebt no und ber wird mir ju mein Recht

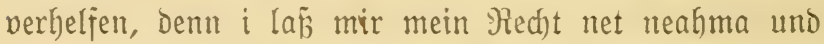
mann i neunmal IAnredst hätt', jo lajiert i mir mei Piecht net neahma, (itreất Dr. 5atichef jeine red)te 5ano ent=

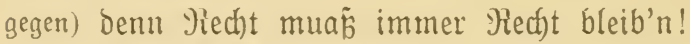

Dr. Şatidjef (ber injwiichen auch aufgeitanden iit, ichlägt in bie ifgm bargereichte Rechte und jagt): Bravo, berr von

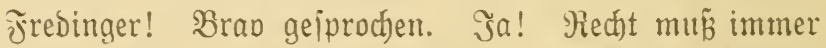
Miecht bleiben! Ias malte (Ssott! (3ur Eeite.) Hracht= volle Rechtsanjofaung! Bei bem fönnte ielbit idh noch in bie Echule gehen. (Worhang jällt.)

Ende Des jweiten Aftes. 


\title{
备rittex dat.
}

\author{
3 wei Jahre ipäter. \\ Fredingers Rontor.
}

\section{Erite Szent.}

Frebinger, Schubiaf und Mitit.

(Jredinger fitst an feinem Schreibtifhe, vorn uto zur Seite fitsen Schubiaf und Miți.)

Jredinger. Allitern is die (S)'idficht fomeit in

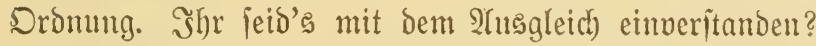

Sdutbinf. Sa, Sdywiegerpapa!

Mitibi. Эa, Nater.

Erdubinf (idnell). Irber wir befommen...

Jredinger (unterbrechend). Siatürli, natürli! So wia ma's vereinbart hab'n. Ind mit die (S'jchroijter Gabt's g'rebt? Dö fan a einveritanden?

Mitit. Die Ioni ja! aber natürlich, Bater, mur fo, wie bu's veriprocken hait.

Jrediuger (beteuerns). § wer do halten, was $i$ amal g'iagt hab. Die Ioni und ibr friagt's a jed's von

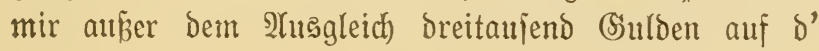


Saand. 2lber wie g'iagt, es berf niemanto was bavon wififen. Und bie Ioni berf unter faner Beoingung er=

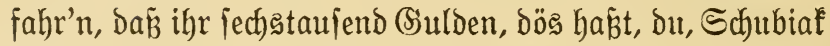
und beine Frau, a jeo's drei Tauiender friagt's. Şr

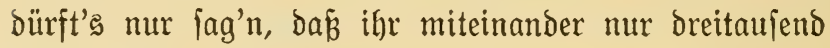
Suldon friagt habt's.

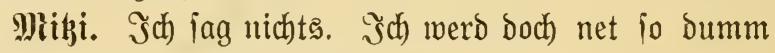
jein uno mein Mann auch net.

Sđfubiaf (lächelno). Îber gar feine ŞDee.

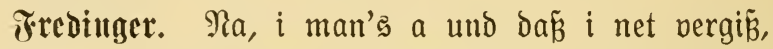

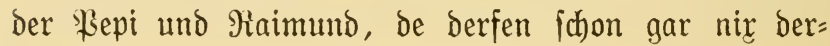
fahr'n!

Sdjubiaf (idnelf). Fäallt uns ein, Schmiegerpapa, wir twerden uns boch nicht blopijtellen und bann würden bie Schröger ja audh fo viel perlangen.

Fredinger. Das funnt $i$ dann net zahl'n, bös jag

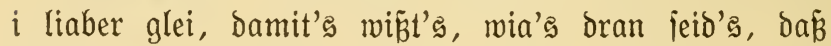
bann net mei Schuld is, wann's de berfahr'n. Der ßepi und der Paimuno, de müif'n fie mit dem $\mathfrak{2}$ tus= gleichsgeld z'frieden geben und wann's net j'frieden ian,

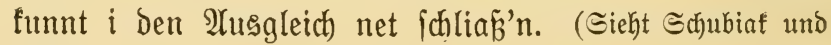
Miţi (auerno an).

Sd)ubiaf. 准er wir und bie Ioni find zufrieden und itimmen zu.

Fredinger. Dös maß́ i, orum jahl' i euch bafür extra.

Edubiaf. Und wann wir zuitimmen, ijt ber $\mathfrak{A} u s=$ gleich geichloffen, benn wir haben bie Majorität!

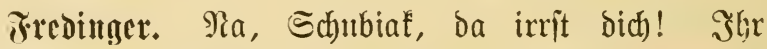
habt's net bie Majorität. Im ganzen fan vier Stimmen. 马Gr jaib's aber nur zmei. 
Edjubiat. Drei!

Fredinger. SMiajo?

Sdjubiat. Şd, mei jrau und bie Toni.

Fredinger (ironifa). Dös fan immer nur zrwa, Denn bu zählít ja net.

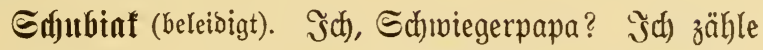

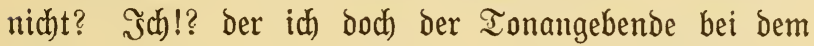
ganzen \$rozés bin. Sch!? ber idh alles gemacht und arrangiert habe. Schmiegerpapa, wenn ind nidjt wäre, fo wäre es überbaupt zu feinem 2 tusggleid) gefommen.

Fredinger. Dös fann wohl mögli fein, aber bas wás $i$ beitimmt, dá̉ bu nichts geerbt hajt und bei

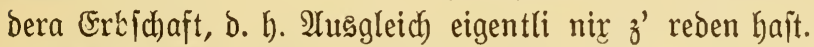

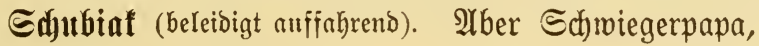
idi) habe bir fo geholfen!

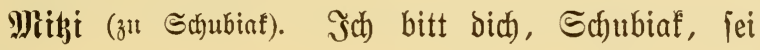
ruhig.

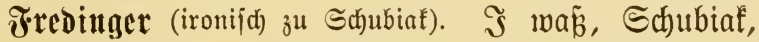

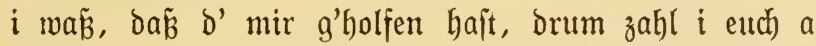
doppelt. Эhr friagt's ia am allermeiften. Itber $z^{\prime}$ reben, b. h. abs'itimmen hat nur bei Frau allan das Recht, dös is mur a Stimm.

Sdjubiat. 2lber ich bin ihr Mann!

Fredinger. Dös is mahr. Das ftell $i$ a net in N(bred, aber Desmeg'n is bös do nur a Stimm.

Mitigi (beruhigeno zu ভ(dubiaf). Der $\mathfrak{B a t e r}$ hat $\Re$ Recht, Schubiaf. $\mathfrak{B i r}$ zwei gelten nur als eine Stimme, benn idh) allein bin nur Erbe, bu net. (马utraulita). Du fiebjit ja, Sđjubią, ber $\mathfrak{B a t e r}$ gibt uns foviel wie für zmei Stimmen. Der $\mathfrak{B a t e r}$ is ja net grausli. 
Tredinger (beiftimmend). Alber na? ? und grausli! gar fan Soee! Dös fecht's ja el), orum lriagt's ifr ja mehr wie alle und jogar boppelt fo viel wie bie Ioni.

Sdubiat. arber fie befommt ja aud) breitaufend (sulden außer ber Âsgleichsfumme und bie Schmäger befommen mur bie $\mathfrak{A}$ usgleichsjumme.

Fredinger. Dös is richti! aber fie is allan a ganze Stimm und hat allan genau io viel Recht beim IItsgleich), wie ihr zma mitanander und trobsem friagt's ihr boppelt fo viel extra als wia bie Ioni.

Sdjubiaf. Das wird fie ja nidbt erfabren. Du jelbit, Schwiegerpapa, hajt ihr gejagt, fie befäme, fo wie meine Frau, bie Miki, breitaufend Şulden extra.

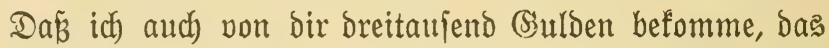
gebt fie ja gar nichts an. Bon mir erfährt bie Ioni nichts und bie Mitisi jagt ihr aud nichts. (Sich an Mitit wentento.) Nidbt?

Mithi (ironifach). Rein, ich werd' ifyr's lag'n!

Fredinger (Iauerno). Es iff mur, baßj fa Durd)= ananoer außzafommt.

Mithi (beteuerno). 2Yber Water, mein Mann uno ich merben boch bir net ichad'n und uns noch meniger. Mie du mit mein Mann zum unterhandeln ang'fangt hajt, hab ich zu ber Ioni g'jagt, dấ bu uns zwa Mabeln einer jeden breitaujend Sulden extra geben willft, wann wir auf ben slusgleich eingeh'n, aber bie Brüber bürfen nichts bavon erfahr'n und fie muaß mit=

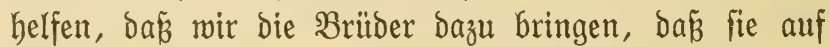
ben $\mathfrak{A}$ tuggleich eingehen. Das war ihr Pecht und pon

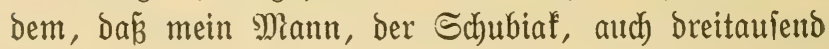


Buldoen friegt, war nie bie Sied' und fie wein aud fein Eterbensmörtl bavon und darf audh nie was erfahren. Sonit iagert's vielleicht gar, i bin a faliche Perion uno Gab's betrogen.

Ednubinf. Das wirb fie nie fagen fönnen, weil fie's nie erfahren wirb. Sie iit mit den breitaujend (s)ulden ertra zufrieden und jitimmt zu.

Fredinger. 2Ulio bös war'n icho zwa Stimmen, de ja lag'n tan. Sas ijt's mit'n ßepi?

Mityi. Der is auch mit babei, er hat ung fachon zug'iagt. Natürlic) friegt er mur bas, was alle beim 2Yusgleich frieg'n und nichts ertra.

Sifubinf. Dem bleibt nidhts anders übrig. Er hat ja gar nidbte zum Reben, er mura, ob er will ober nicht.

Mityi. (Sar io einfach war bie (s'jichidyt net. Die Ioni uno id) bab'n ifn ordentlich bearbeiten mülifen uno bie Ioni hat ifm gottsjämmerlich vorlamentiert,

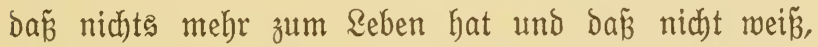
was anfangen foll, wann ber 2 usgleid) net zuitande fommt.

Sdubiaf. Brauchen tut er's aber notrendig, benn er pumpt ïberall herum.

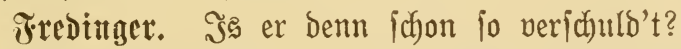

Miini. Biemlidh, er hat net gleich nadjgeb'n woll'n. Es war ilym z' wenig, aber weil wir ifm gar fo zus g'rest hab'n, fo hat er halt g'iagt es ifit ifm recht und er geht allf ben 2 tusgleich ein.

Fredinger (zufrieben). 2Ulfo dös war'n icho brei.

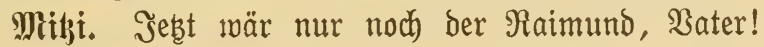


Frediuger (auffahreno). Zu was den? Den brauch'n ma net.

Edjubiaf (zuĩtimmend). (šanz ridhtig, ভdhiviegerpapa, ben braudhen wir nidht zul fragen, den eingebildeten Sausbuben, ber ift minorenn und hat nidfts zu reden.

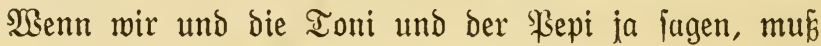
er, ob er will ober nicht!

Fredinger. 2uber ber wird ja gar net g'fragt. Warum benn net gar.

Sd)ubinf (veractungsvoll). (Sanz natürlich). So mas merben mir audb nod) fragen.

Mitij. Es is net io fehr um den Raimuno, als wie wegen ber Bormunojchaft.

Freditger. WMas, megen ber Sormund \{chaft?

Mitipi. Es ift nur, meil fich ber Dr. Jeger eben wegen ber Bormundid)aft ipreizen fönnt.

Jredoinger (Iauerno). গa, fo hätt's halt mit'm Dr. Jeger g'reot!

Mitji. Das haben wir eh getan, wie wir wegen Dem Aluggleich g'redt hab'n.

Fredinger. Dann ift's eh guat! Mas hat er benn

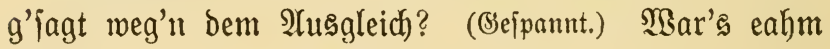
recht?

Mitji. Die Toni und ich maren brinn bei ifym und hab'n ihm g'fagt, dẩ mir alle net mehr anders ausfönnen, weil wir von ber Şand in ben Mund leb'n. 's ift ja bie reine Mahrheit!

Sdjubiat (einfalfent). כ̧hr habt ihm aber gejagt,

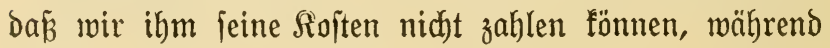


bei 3uitandefommen Des 2 Uuggleiches der Sduriegerpapa auch leine Siojten bezahlen wirb.

Mitji. Sa, das hab'n wir ihm alid) g'jagt!

Frredinger (höhnifđ lächelnd). Dann war's eafym recht?

Mitji. Er hat überbauñt net nein g'iagt. Er hat nur g'meint wegen bem Raimumo, weil ber noch net groşiährig iit, wegen ber Bormunojdaft, da hat er g'iagt, oa is er verantwortlich.

Fredinger. Alber gar net wahr! Der eigentliche Bormund bin ja $i$ und menn ifr zum $\mathfrak{A}$ usgleich ja jagt's, io ...

Mitji. Das hab'n wir dem Dr. Šeger eh g'iagt.

Fredinger. Und dann hat er an Muah geb'n?

Mitji. Er hat nur g'iagt, er will feine unan= nehmlichfeiten haben megen dem raimuno, denn Der Raimund ift ein Schreier und es wär' am beiten, wenn wir mit dem Raimuno jelbjt redeten und ihn aud bazu

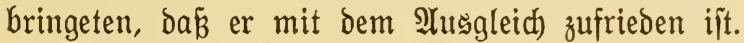

Fredinger. Shr merd's do net gar mit bem Raus= buab'n zum unterhandeln ang'fangt hab'n?

Mitipi. Unterhandelt hab'n wir net.

Fredinger. Mas denn?

Mipi. (S)'redt hab'n wir mit ifm.

Frebinger. $\Im \mathfrak{s}$ eh das gleidhe. $3 \mathfrak{u}$ was benn mit dem Rausbuab'n red'n?

Sdubiaf. (Sanz natürlich, ber Schmiegerpapa hat recht. Der muś den 2̂̉sgleich annefmen, wie er ijt. Fredinger. Wer hat benn mit eahm g'rebt?

Mithi. Die Toni uno ich hab'n mit ifm g'rebt. Fredinger. Dein Mann net? 
Mligi. Mein, Denn mit mein Mann reot er nichts. Sdjutat. Jrecher Rausbub!

Fresittger (neugierig). Was jagt er benn zum Itusgleicf)?

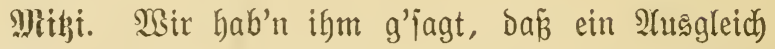
geichlofien werben foll, ba bat er gleich g'fragt: Miejo? Jredinger (geipant). Şhr habt's ifm do net g'iagt, dá⿱㇒㠯 er meniger...

Mithi (einfalfend, idgnell). Slein, nein, Bater, bas haben wir nicht getan. Er weif nichts davon. Es gefyt ifm felbit jefr ichlecht, aber er fublagt fich burch und mird barum um fo bocfbeiniger. Da muás man mit ifm iefre vorfichtig jein. Mie er g'fragt hat wiefo, ba haben wir ihm zuerit vorlamentiert, ba (c)lecht gebt, baß mir net amal mehr recht z' efijen

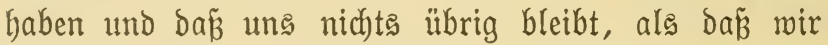

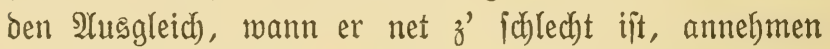
und mit den zweitaujend (suulden vom 2tuggleid) zu= frieden fein müijen. Das war ifm z'erit net recht und er hat's net annebmen mollen, da haben wir ifm zu= g'redt und hab'n ihm g'iagt, baß ser ßepi aud babei is und er wird's uns dod) net verderben.

Jredinger. Da hat er dann nachgeb'n?

Mithi. Scin und her ijt's gegangen, Dann hat er g'iagt: J̧⿻ bin minorenn und fann nicht viel machen.

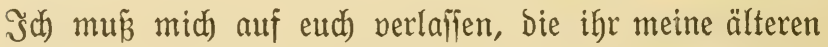
(şefdymifter feid, und mann ber \$epi aud babei ift, wirb er icjon fehen.

Edjubiaf. J゙h begreife nicht, Mibzi, was für (Sse= fdichten ifyr mit bem Raimund madht, ob ber freche 
Shus will oder nicht, bas ijt ganz Rebenfache, er ijt minoerjährig und lyat nichts zu reden. Mag wir be=

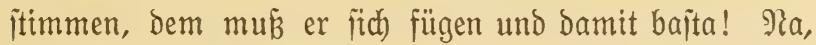
Dem möcht ich's jeigen!

Fredinger. Mann ma funnt. Ta, der Rausbua wird ipiben beim 2 (usgleich, was er friagt, ber fann fich Den Minno abriijchen.

Mithi. Stber Bater, es ijt net fo einfach.

Fredinger (Frech). Megen mas Denn?

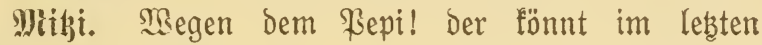
Mioment z'rücftreten.

Tredinger. SBarum Denn?

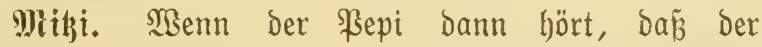
Raimund, wie ber Bater fagt, fich Den Mund abwifden fann, fo wiro's ifm nicht recht jein.

Sdubiaf. Faperlapap! Der \&epi ift froh, wenn er jein şeld erbält.

Fredinger. $\Im$ man's eben $a$.

Mitji. Arber ber \$epi glaubt, dá̃ Der Yiaimund foviel wie er befommen foll.

Fredinger. Das friegt er a.

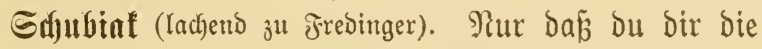
Şälfte bavon gleich) zurüdłbebälit.

Irredinger. Эaa, für feine Erziefung.

Safubiat. Und bie andere Şälfte?

Fredinger. Die heb' $i$ ihm auf, bis er 24 Jahre alt wor'n $i$.

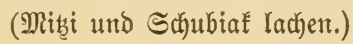

Sdubiaf. Pa, uns fann's recht fein unt ber $\mathfrak{P e p i}$ wirb auch nicht mehre viel baran ändern fönnen. 
Mlifi. Denn er ift jo notig beinand, daj̉ er gar nicht mehr aus fann.

Sdjubiaf. Siun, dann wirb's (chon geh'n.

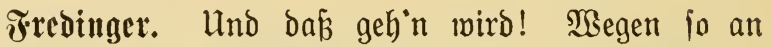

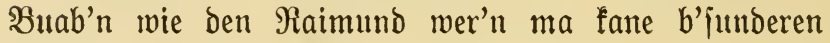
Ianz aufführ'n. Frelien muaź er's, ob er will ober net. (Sieht auf feine $\mathfrak{u g r}$.) $\mathfrak{U} \mathfrak{m}$ a zehne fommt mei Doftor. (3u Miţi und schubiaf.) Ese fan do alle ver=

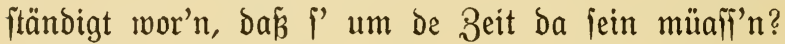

Mithi. Saa, ater, all'n hab'n wir's g'iagt. Der Ioni hab' ich g'fagt, fie foll frïher fommen, bamit fie mit bir noch abmadjen fann. Sie mú jeden Moment fommen, benn wir hab'n ung abg'redt, baj̉ wir fo a viertel Stund früher da z'fammentreffen, aber ich bin mit mein Maann eigens noch früher fommen, bamit auch) bie nidbts fieht, wegen mein Mann und ba mußst fđhon entichuldigen, ßater, wenn ich bich bitten tät megen bem (šeld (gibt Schubiaf einen $\mathfrak{B i n f}$ ).

Sdjubiaf. Es märe gut, Shwiegerpapa, menn Sie uns bas (Seld gleich) geben würden, bevor bie Ioni noch fommt.

Jrediuger. STlber zu was benn? Preffitert's denn fo? Nadjher gib $\mathfrak{i}$ eud's.'

Mitiji. Arber Bater?

Sdjubiaf. Warum denn veridhieben, Schmiegerpapa?

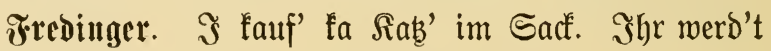
es idfo friag'n. MSas $i$ veriprich, bas halt $i$ a!

Sdjubial (fid) ereiferno). Mein, Schriegerpapa, auf bas geh' ich nicht ein. 
Fredittger (Iauerno). 2lber ihr fönnt's do wart'n bis muaring. I hab' bas (Seld net ba.

Mithi (zögerno). Mann's mein Mann - -

Sdjubiaf. Iut mir jehr leio, Sd)wiegerpapa, aber ohne Bseld fein 2 husgleich!

Frebiutger. Du brautchit mir net glei ben Fienolver anies'n!

Sdubiat. I I idy aud nicht, aber lieber Schwieger= papa, (Sejchäft ijt (Sejejäft!

Fredinger (fich wehrend). Эch hab' aber net fo viel ba.

Sdjubinf. Da fann idh nicht helfen, idficf' in bie Bant.

Fredinger (mürrifch). Na, fo was, an jo z'oräng'n, als ob $i$ a (sauner wär. $\Im$ geh ellch ja net burch.

Mribi (zutunlich falich). Water, bu entichuldigit ichon, aber ....

Fredinger (mürrif und zornig). $\Im_{\mathfrak{g}}$ idho guat! (Denft ein wenig nach und jagt bann.) I mir nachichau'n, ob i's hab. (Steht auf und geht zu feiner eifernen Raije, öfint bies jelbe, entnimmt Derjelben, von Beit zu 3eit vorjichtig und miß̄s=

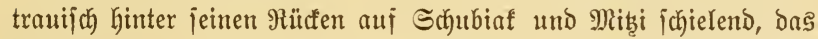
Beld, fperrt bie אaffe Giernuf zu und fommt mit bem Belde in ber

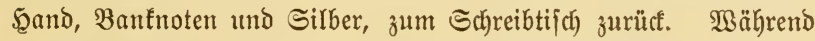
er bies tut, führen Miţi und Schubiaf folgendes Beipräch.)

Mitji. II hätteĩt Doch nicht io orängen foll'n!

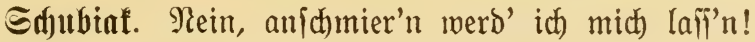
Mithi. Bielleidst hat er's boch net ba?

Sdubiaf. Şh mödte das haben, was er ba hat in Der Saifie, ber alte (S)auner!

Mithi (abwehreno). Nidcht io laut, jonit hört er bid). Sdjubiaf. Der glaubt, er fangt midh. Utber geh, 


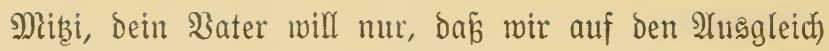
eingeh'n und dann fönnen wir uns das (SEeld fuchen. Midh fängt er aber nicht, benn ich habe es ichon mit geriebeneren Saaunern zu tun gehabt und habe nicht umionjt jahrelang im Drient gelebt.

Mithi (gibt ifm einen $\mathfrak{B i n f}$ ). $\mathfrak{s} \mathfrak{f t !}$ ! er fommt.

Fredinger (zurücffommend 孔u Schubiaf und Miţi). So, i hab das (Sield g'rab no g'funden. Es g'lengt auf a Şaar. Drum mü̈t's a a Silber nefmen, denn $i$ hab alles z'iamm trats'n müaif'n.

Edjubiat (Iäthelnd). Sh! Schriegerpapa, wir nehmen alles.

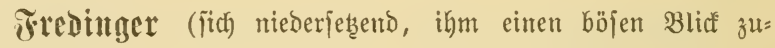

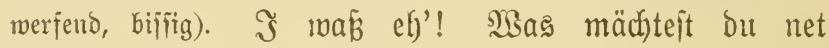

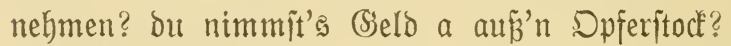

Sđjubinf (Yiebensmürbig lächelno). Und du etwa nicht, Schwiegerpapa?

Fredinger (zornig). Beleioig' mi' net, Schubiał. $\Im$ bin an ehrlicher Mann.

Mibi (interveniereno zu ভajubiaf). YYtber Schubiaf, jei Doch) rufig.

Edjubint (lädjeIno). Sît fhou wieder gut. Es war nicht io id)limm gemeint; Schrwiegerpapa wiro's nid)t fo bös aufnehmen. (Siebensmüroig zu jresinger.) Richt wahr, Schwiegerpapa? Silchts fïr ungut?

Fredinger. Sficho recht!

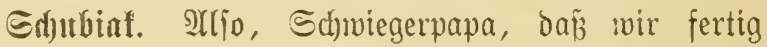
werben, bevor bie anderen fommen, (iiegt auf jeine $\mathfrak{u h r}$ ) es ijt nicht mehr viel Beit zu verlieren, jei jo freundich und gib das (S)eld her. (5ält ihm bie offenc Şand entgegen.) 
Fredinger (zögerno und mürrifक). SSlei! eS brennt

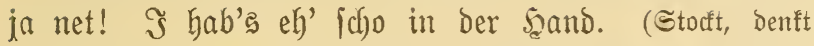

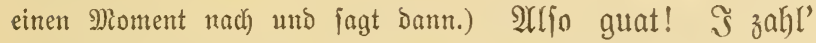
euch) das (sjelo jebt aus, aber igr mitafit's mir be=

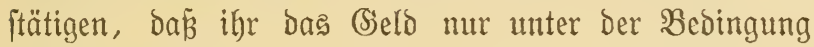

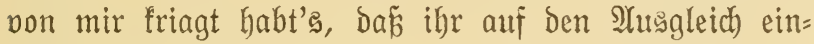
geht's.

Sflubiat (auffpringend und abwehrend). Sieiu! bas geht abjolut nicht.

Mitji. Bater, bas fönnen wir net tun!

Frediuger. Warum Denn net?

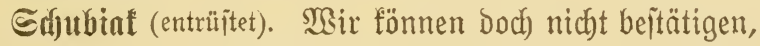
dẩ wir Seld dafür befommen haben, damit bie anderen betrogen merben.

Frediuger (entjejieden). Da fann i baun a net. Shne Beptätigung fa (Sield.

Sdubial (erregt). Atber Sdhwiegerpapa, wir find ja leine Schininder.

Frediuger. I a net. Whie bu mir, fo i bir!

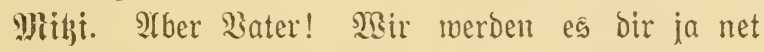
abitreiten!

Jredinger. Dös fann ma alauben uno a net.

Mitji. Water!?

Sdubiaf. Schwiegerpapa!?

Fredinger. Iraut's ifr mir net, traut i ettch a net!

Sditubinf (heudhelns). Schwiegerpapa, wir haben fein Miñtrauen gegen oich).

Fredinger (jarfajtifich). Ra net! Aber's (selo mollt's hab'n, bevor ber 2tusgleid) abg'ichloil'n is uno mir mollt's net beitätigen, da 
Sr)ubiat (ichnelf). Das bejtätigen wir bir, Schwieger= papa!

Jredinger (mis̄trauifa). Mas?

Edjubiat. Daß́s mir auf den 2 Huggleich) eingeh'n. Fredinger. Und's Sseld?

Sdubinf (zögerno). Weiß̄t, Sdwwiegerpapa, bas laffen wir vorberhand.

Frediuger. $\mathfrak{A}$ na! MBia g'lagt, ihr bejtätigt's

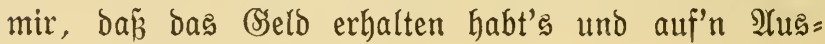
gleich eingeht's.

Edjubiaf (itellt firch mit zornig emporgehobenen ફ̧änden vor Fresinger). 2ther Schmiegerpapa, wie fannit bu mur io

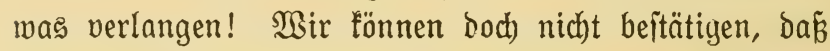
wir ung beitechen haben lafien und baj mir mehr be= fommen haben als bie sefchmitter. Es ift unmöglich. Sch lann bas idfon gar nidht. Millit bu benn bas nicht begreifen?

Mithi (in befhwötendem Zone). Bater, bas geht nicht, bas fönnen wir nicht!

Fredinger (iich) Dumm ftellend and mit Miţi und Schubiaf wie bie Rnţe mit ber Maus fpieleno). $\Im$ begreif' eud wirfli net. Şhr friagt's das (Seld do nur unter ber Bee bingung, daß̉ ihr auf ben 2 (uפgleich) eingeht's und wollt's das net beitätig'n. M̉arum benn net? Ş ja gar nichts dahinter! Mas tua $i$ Denn, wann ifhr bas Seld ichon habt's und ifhr ïberlegt's eud bann und jagt's bein $\mathfrak{i}$ (usgaleich) na.

Sdubiaf. Itber Sdmiegerpapa!

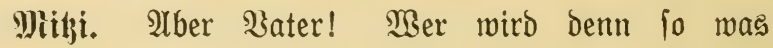
Denfen! 


\section{$-97-$}

Sdubiaf. Shrwiegerpapa, wir werben bod) fo mas nicht tun.

Fredinger. Son mir verlangt's aber, i joll euth bas (Seld por abgeichlofienem 2lusgleid) geb'n? (Bes toneno.) Marum benn bei mir utto net bei end)? Bin i vielleicht gar a (d)lechterer Menidh wie ihr? $\mathfrak{J}$ bin an ehrlicjer Mann und redhtidbaffener (Sharafter und hab no neamo toas g'numa!

(Schubiaf und Mitifi fehen iith) gegenjeitig verbuzt an uno bleiben ftumm.)

irtedinger (bie Situation ausnüßzent). Da bleibt's itab, da mifrt's fa 2 tntwort und is do ganz der gleiche Fall, wie bei euch. Şhr mollt's net auf ben Itrsgleich ein= geh'n, bevor $i$ euch net bas (S'eld geb'n hab und $i$ gib euch) net bas (Sseld, bevor ihr net bejtätigt's, da a i hr Dns (Sield friagt habt's, Damit's auf den Âtugleich eingeht's.

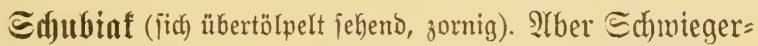
papa!

Frrdoingcr (zeigt auf Papier und Feber, Gält ifuen bas Bield

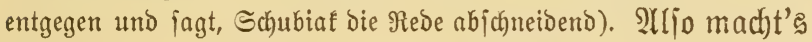
weiter fane (S)'ichichten! Ia iit Papier, Iinten uno Feder, unterichreibt's uno da ijt das Sseld! (Rleine \{raufe, baun in fragendem, aufforberndem zone.) SIlfo?

(Schubiaf uno Mititi jehen jïch ratlos an. Schubiaf icharrt zornig mit Dem ริuñe.)

Fredinger (wiederfolt betoneno). 2llio! Entweder io oder fo! \$ fann net an Emigfeit wart'n und bie andern wer'n a glei ba fein. 
Miipi (erichrocten zu Schubiaf). Es ijt mahr, Schubiaf, die mürī'n gleich da jein und fie bürfen nichts wifīen.

Edjubiaf (melcher vor fich) hingebrütet hat, finnt auf bie $\mathfrak{A} \mathfrak{n}=$ rese jeiner Frau einen Mioment nach und jagt dann zu zresinger).

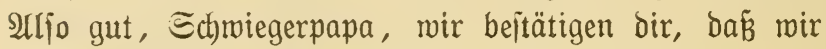
auf ben susgleich eingeh'n merden und (z̈̈gerno).

Frediuger (iđnell, lauerno). Und's (șeldo? Das fönnt's mir abjtreit'n.

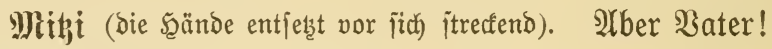

Frediutger (mit Bsenugtuung). Şätt's net ang'fangt.

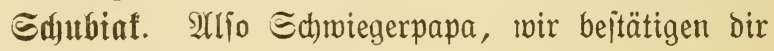
auch ben Empfang bes (Sieldes.

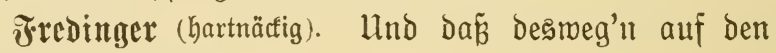
2Uugaleich eingeht's.

Edubiat. Siein, bas geht nicht.

Fredinger (idnefl). Dann fann $i$ a net.

Sdyubiat (in heller 2 (ufregung). SYber hör, Schmieger= papa, la

Fredittger (gejpannt). Na?!

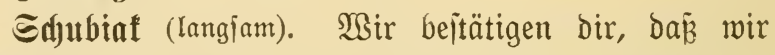
fech)staulend (Sulden erbalten haben (Wort für Wort be= tonend) auf einem 3ettel.

Fredinger (geipannt und miştrauij(h). Und?

Sdjubiaf. Und auf einem andern Bettel beitätigen mir, baż wir auf ben 2 Uusgleich eingeh'n.

Miji (idnell und freubig einfallend). Ja, Nater, jo geht's!

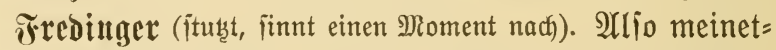
meg'n! Şhr beïtätigt's mir auf einem Bettel bas Seld und auf bem anderen 3ettel, baß́ ihr auf ben 2 huggleich eingeht's (unterbricft fich). Dös hẩt, eingang'n feios. 
(Schubiaf will proteítieren und hebt bie Sand als Zeiden ber 2(bwehr.)

Mliłji (winft ifm ab). Das fönnen wir tun, Scbubiaf.

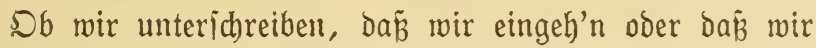
eingegangen find, bas bleibt fïch ichliep̧lich für uns egal.

Sdubiaf. Das glaubit bu!

Misi. Arber Schubiaf, bamit wir fertig werden. Fredinger. $\Im$ man's eben $a$ !

Sabubiat. Meinetwegen (ergreift die žeder, fabreibt zu= erit einen Bettel und zeigt ifn jresinger). Das ift bie $\mathfrak{B e}=$

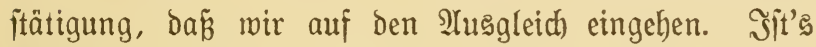
dir jo recht, Schmiegerpapa? \$Benn ja, bann merden wir ifn unteridreiben.

Frediuger (liejt ifn und gibt ihn zurücf). (S)uat jo!

Edjubiaf (nimmt ben Bettel, unteridgreibt ing und ichiebt ifn

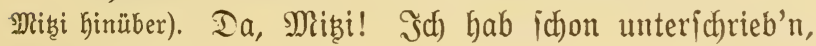
unterfacreib bu!

(Miţi tut fo und während Schubiaf an Dem zmeiten Bettel Der

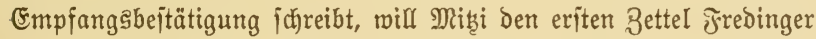
geben. Diejer will ihn idjon nebmen.)

Edjubiaf (bies bemerfeno uno idnnell den Bettel Miți aus ber 5̧and nehmend zu Frresinger). Du entichuldigit ichon, Edbriegerpapa, aber alles miteinander.

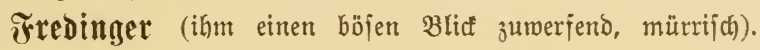
Meinetwegen, aber tummel bich!

Edubiaf. (S)leich bin ich fertig, Schriegerpapa! (Sd)reibt Den zmeiten Better fertig, unteridreibt ifn und gibt inn Miţi.) Da, Mişi, die Empfangsbejtätigung über das (Seld. Э̧ch hab ite gleid) unteridbrieb'n. Interidyreib bu!

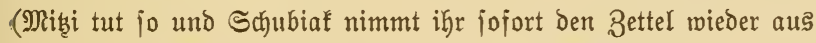

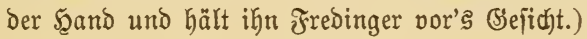


Sdubiaf. Da, bie Empfanģbejtätigung ïber bie fechstaujend (Sulden. WBir haben fie gleidh unterfarieben. Sit fie bir fo recht?

(Frebinger liejt ben Bettel, wifl ihn nehmen, aber Schubiaf zieft ¡chnell bie 5̧and zurücf.)

Fredinger (zornig). Alber fo gib ihn do her!

Sdjubiat. (S)leich, Schroiegerpapa. Sijt er bir jo recht?

Fredinger. Э̧ icho guat (will ifn wieber nehmen). (Sib inn her und den alloern 3ettel $a$.

Grubiaf. (S)leich! (itrectit ifm bie andere 52and entgegen). Zuerit bas (Seld, Echmiegerpapa!

Fredinger (muiteno). Ma da hört fi do alles auf. Mir fan do in faner Mauberhöhl'n. Sebtn lern $i$ euch erit mirfli femua. (Sib den Dredf non be Betteln her und ba habt's es Sseld.

Edubinf. Einen Moment, Schwiegerpapa. (ङibt Miți einen Bettel.) So, Miķi, das ift bie Empfangs= bejtätigung über die ganzen fechstaujeno (Sulden (Sib ou fie dem Schmiegerpapa uno er foll bir beine orei= tauleno (s)ulden geben und ich, (zu orresinger) Schinieger= papa, gebe dir bie Beftätigung, daß̧ mir auf ben $\mathfrak{A}$ gleich eingegangen find, gegen meine breitauieno (Sulden. ?idht wahr, Schwiegerpapa, lo geht's?

Frediuger (zornig und mürrifd). Sa meinetwegen. (s)ib her, Sdubial.

Sdjubiaf. Mein, ভdniegerpapa, die Miki juerit!

Frredinger (Schubiaf einen böjen Blidf zumerfenð). Maarum Denn die Mibi z'erit und liet du? Das bleibt it ja ganz gleich. 
Sdulbiat. $2 \mathfrak{A}$, Sdywiegerpapa, oa irrit bu bich, bas bleibt fich nicht gleich).

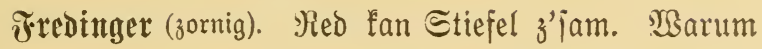
joll î benn bas net gleid) bleib'n?

Sd)ubiaf (böflich, ironifa). (Sanz einfach) aus folgendem Sirunde, lieber Sdrwiegerpapa. MEenn bu bei mir ben Infang machit, jo hajt du bie 3ettel daritber in ber Şand, dẩ mir auf ten 2lusgleich ichon eingegangen find und wir hingegen haben erit bie Saälfte des (S)eldes, ๖. h. Dreitaujend Ssulden in Der Şand. Die Seitätigung über bie ganzen jectstaulend (S)ulden brauchit bu bann nicht mehr, denn ou hait uniere idhriftliche Ilnnalyme Des 2 usgleiches bereits in Sänden und eriparit bir auf bieje Meije die zmeiten oreitaujend Sulden.

Fredinger (wild aufipringend). Das geht zll meit. $\Im$ bin ja la Rauberhauptmann.

Mitji (interveniereno zu Sdjubiaf). 2rber geh, Schubiaf, rie fann man benn nur gar io jein?

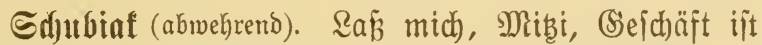
(Sejchäft! (3u srebinger). Du nimmit mir's nicht für übel, Sdbriegerpapa. Menn bir hingegen meine Frau zuerfit ifren 3ettel gibt gegen deine Dreitaujend (Sulden, fo hajt bu wohl bie Empfangsbejtätigung über bie ganze Summe von jechstaujend (Siulden in ber 5̃and, befib'it aber nicht bie Errflärung, dẩ wir auf ben 2lusgleich eingegangen find und verbleibt uns fomit noch volle Bemegungsfreibeit.

Fredittger (bififig). Sor euch) mua ma fi hiuten!

Edjubial (berufigend). 2lber warum Denn? Das werden wir ganz einfach machen. (马u Mitizi.) Du, Mik̨i, 
gib dem Schmiegerpapa mit ber einen Şand den Bettel und mit der anderen Scand übernimmit bu bas Sseld, melches bir bein $3 a t e r$ gleichzeitig ïbergibt, und idh mache das gleiche.

Fredinger (unwirich). Allio mach ma amal an Enb! (Sebt's her den Drect und da habt's es (Sield.

Sdjubiaf (recht höflich). \$arbon, Schrwiegerpapa, eine Jrage. Stimmt das Selo? 5̧ajt bu es gezählt?

Frredinger. (Ssanz natïrli hab i's zählt, twia i's aus der Siaifa g'mumma hab. (3eigt auf bas vor igm liegende Bserdo.) (5is jan genau jectstaujend (Sulden.

Sofjubiaf. Bitte, Schmiegerpapa, es nachzuzählen. Fredinger (mütend). Das is ma z' bumm! is bin ja fa Sdmmindler! S bin an ehrlid)er Mann und a rechtichaff'ner (Eharafter. $3 \mathfrak{u}$ mas denn nad)zähl'n? Es jitimmt a io a.

Sdjubiaf (iegr freundlich). Nichts für ungut, Echmieger= papa, aber ficther ifit ficher.

Fredinger (böie). Na, bie (s'; 'ichichten! Mneg'n fo an Drect (zähit).

ভdjubiat (wie Freoinger bei breitaujend anlangt). So, Schmiegerpapa, das mären bie breitaujend (Sulden für bie Mithi und jekst meine oreitaujend Sulden.

(马resinger willfahrt und zählt bie zmeiten breitaujend (sulden ab.)

Fredinger. Das fan jeşt beine breitaujend (S)ulden. (Es liegen jeţt zwei מ̧äuflein von je breitaujend Buldben vor

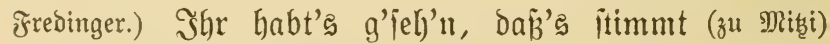
und jesten gib mir bein Bettel her und da hajt bas (seld. Mithi. Da, Bater! (übergibt æ̌resinger mit einer Şand 
ben Bettel und übernimmt mit ber andern bas ihr von ifm bar= gereichte (Seldo.) Tante, Bater!

Fredinger (zu ভchubiaf). Da hait du deine brei= taujend (Siulden und gib mir dein 3ettel.

Gaubiaf. Bitte, Echmiegerpapa. (Ier gleiche ßor= gang wie bei Mitzi miederholt fith.) Ianfe jehr, Edymieger= papa!

Fredinger. Sebet zählt's ifhr nach, ob's itimmt.

(Miţi uno Schubiat tun jo.)

Mitij. Šit ichon richtig, Water!

Edubiaf. Stimmt nollfommen.

(Miţi und Schubiat find in Begrifie, jedes jein Bseld zu verjorgen, als geflopit wird. Sie fahren zujanment.)

Jrebinger (leife). YYGer i hab ja zug'riegelt, wia ifr fommen jeio's. (Raut.) Maer is?

(Man hört, wie braupen verjucht wirb, bie Türe z̆u öfïnen und hört bie Stimme von Toni.)

Toni. Sch bin's, Bater! bie Toni.

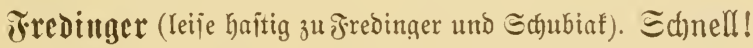
ichnell! pact't's z'iamm. Die Ioni is.

(Miţi und Schubiaf fitecten in aller Şajt bas Gielo ein.)

Fredinger (Iaut zur Türe hin). $2(h$ jo! bu biît's, Ioni. (3)lei mach) $i$ auf.

Mithi (erifhrofen). Das is mir aber unangenehm, baṕ uns bie Toni ba fieht, no Dazul eing'iperrt, was

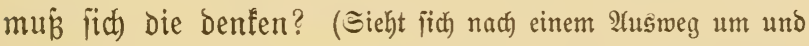
bemerft bie Türe, bie ins fontor führt. Seife aufïteheno zu Fresinger.) Du, Bater, wir geh'n ba naus (zeigt auf bie Rontortüre) und fommen bann nacbher von ber andern Seit'n wieder rein. 
ซredinger. Ssuat is. (Sieft auf bie Mhr.) 2lber be= fitimmt um zefne mieda ba fein.

Mitji. Sanz beitimmt, Bater. Und mann bie Ioni fragt, fo jag ihr, wir war'n ichon ba und hab'n auf fie g'wart und find bann wieder fortgang'n.

Fredinger (Leije). Э wir's ausricht'ı. Inmmelt's euch.

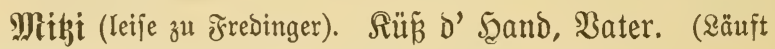
leife mit ভchubiaf burch bie Rontortüre Ginaus.)

Fredinger (riegelt Ginter ifnen ab, läuft Gieraụ zur mittleren Türe, riegelt diejelbe auf und öffnet jie). So, Ioni, jebt fannit icho eina.

\section{3weite Şene.}

Frebinger und Toni.

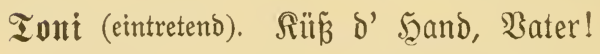

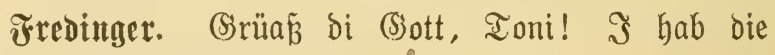
Iür zuag'riegelt g'babt, weil ' $i$ g'rad o'ran war, bei (Seld g' richten.

Toni (bemertt bie vor und neben dem Schreibtifd) Ftehenden

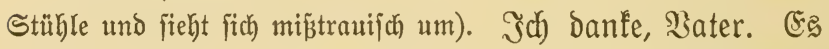
prefifert eh fdion, denn der $\mathfrak{B e p i}$, der lann alle Moment fommen. Sch hab'n von der Meiten g'ief'u und bin

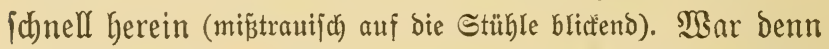
bie Mikzi no net da? Sie hat mir g'jagt, i foll zmijchen halb und oreiviertel Befne herfommen, bamit wir wegen bem (Seld noch mit bir abmachen (jeţt fich nieder). Du erlaubit ichon, Bater?

Fredinger. Seb̧ di mur, Toni. (Err fetet fich ebenfalls.) 


\section{$-105-$}

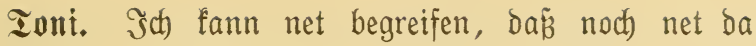
fein jollt.

Fredinger. Sie war eh fchon vor ungefähr aner halben Stuno ba utlo hat lang auf di g'wart und weilit aber net fommen bijt, hat's g'jagt, iie geht jebt und fommt uma zehne wieder.

Toni (ichnefl). Ia war's ichon ba? Saajt bu's fabon auszahlt, Bater?

Fredinger. Du manit bie breitaujend Ssulden? Sa, be hab i ifr geb'n.

Ioni. Dann fönnt i's vielleid)t aud gleich habn?

Fredinger. Utber ja, Toni. $\Im$ war eh g'rad d'ran, bir's z'iammz'richten. Wart a biffl, i Gol's nur aus ber Salja. Sannit mir dermeil bie Beịtätigung idreib'n. (Geht zur Rafle, öffnet biejelbe und will ihr bas G̈eld entnefmen.)

Toni. Sa, Bater, gleid). (Sie will bie Feder er= greifen, als fie auf bem Schreibtif bie Bettel liegen fiegt, welche Fredinger in Der byajt megzuräumen vergeffen hatte. Sie blid̛t zu= erjt auf Den einen, bann auf den zmeiten, fäbrt fojort barauf los und nimmt fie in bie Şand; in zornig eritauntem Ione.) Эa, was î́t benn bas, Bater?

Fredinger (ahnungstos in feiner אaffie framend). M̉as Denn, Ioni?

Toni. Du hajt ja ber Mibi und bem ভchubiaf lec)staufeno (sulden miteinander geben!

Fredinger (breht fid bei der Raffe bliţignell um). MSer jagt bas? (Wie er bemerf́t, daß̉ Toni bie Zettel in ber F̧and hălt, rennt ex exjdroden zu ihr uno will igr biejelben megnefhmen.)

(Toni meicfit aber aus.) 
Fredinger. Ssib mir meine 3etteln, de geh'n di nir an. Dö jan wegen an andern Seld.

Ioni. 2lber lüg boch net, Bater! ber eine Bettel ift die Beittätigung von ber Mib̧i und ifr'n Mann,

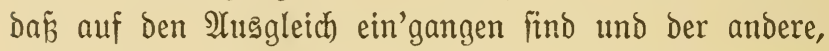

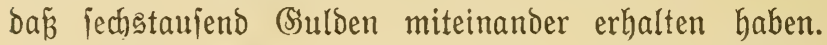
Beibe find von ber Mikisi und vom Schubial unter= idfrieb'n und's Datum von heut ift auch brauf. (Sreift.) Uno feucht find's auch noch. Arlio gibt's nichtes mebr zum ableugnen, Bater!

Fredinger (zornig brängend). Das geft bi gar nix an, was brauf iteht, dös fan meine Betteln uno g'för'n mir. 乡llo her damit.

Tont (immer zorniger werbent). Ath! fo mas foll mich nichts angeh'n? Mefr mie jeden andern. Srieg'n bie zmei jechstaufend (Sulden miteinander! Maser hätt fich das benten fönnen. Mich fo z' betrügen. YUs ob bie mebr Recht hätten, wie idh. So a Semeinheit! Der Sdubial hat ja nichts geerbt und hat brum auch nidbts z' reden, hat aber aud Dreitaujend (Sulden friegt. Niederträchtig! Drum ift die Mibi frïher fommen,

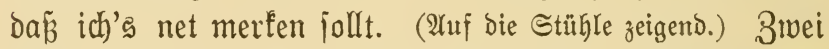
Sefiel find aud) ba und find dag'ĩtand'n, als ob g'rad jemand bavon aufg'îtanden wär! 2ltha, jeşt begreif ich aud), warum's zug'iperrt war, wie id) an'flopft hab, benn da waren's g'rad da (iiefft fith um und ihr Blicf bleibt auf Der Bureautüre haften) und da find's 'naus (zeigt auf bie Türe uno will hin). So eine (Ssemeinheit! So eine Niederträcútigf́eit! Meine eigene Schmeiter und midh fo zu betrügen. 


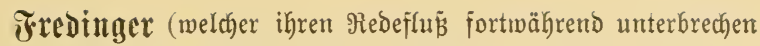
wollte, tritt ihr nunmehr in den $\mathfrak{B e g}$ ). Mach ma fane Manderln, Ioni und gib mir meine Betteln z'ruct. $\Im$ fann tuan und mach'n was $i$ will und wann bei Schmejter a Sinalli und ber Schmager a Saberlump

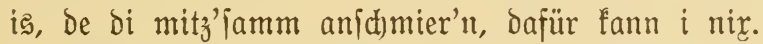

Toni (zornig und ichreiend, weineno, unterbriçt ifn). So, Bater, dafür fannit du nichts, mannit denen boppelt fo viel gibjit wie mir! Für was benn? Saben denn bie mehr Recht wie ich? Reine Spur bavon, denn bie Mibi hat af'rat io viel Mecht mie idh und ber હchubiaf, ber hat gar nichts z' reben, ber mifcht iich nur ïberall hinein, mo's ihn nichts angeht. Für was friagt benn ber a breitaufend Sulden, für mas Denn, Bater? Doer hab ich bir meniger g'holfen, als wie bie Mitibi. (Bes teuerno.) Mann ich net bin, bie bem \$epi jo zug'reo't hätt, jo giengert ber ficher net auf ben 2 (usgleich ein uno ber Maimuno jujon gar net.

Fredinger. Alber mach do net gar fo an Sramall!

Toni. Soll ich net an Sirarall mach'n, mann man

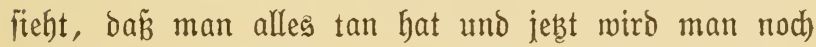

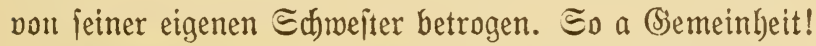

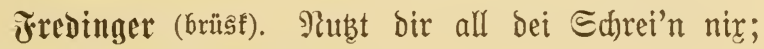
das is net mehr zul ändern.

Toni. SBeil bu's ihnen hineingejtect't hajt, too bu

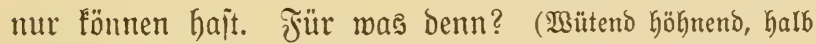
befinnungslos.) Daß́ bich Gint' und vorn ang'ichmiert hab'n! Ia ́a dich beim Ieitamentmach'n von der Mutter betrogen haben!

Jrendinger. Mit!! 
Ioui (milds). Ja, bich), Bater, ober ijt das fein $\mathfrak{B e}=$ trug, wann's a faliches Teitament unterichob'n hab'n?

Fredinger (auffahreno). $\mathfrak{B a s ~ j a g i t ? ~ ( \Im ̋ a ̈ h r t ~ a u f ~ I o n i ~}$ 子u und pact fie bei den Şandgelenten.) $\mathfrak{A}$ falich Ieitament bab'n's untericboben?

Toni. Sa, a falich Teitament hab'n's unterichoben und ber Dr. Jeger hat ifnen g'holfen.

Fredinger (in fürchterlidjer 2 (ufregung, brüct ihr bie Bselente

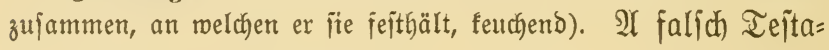
ment hab'n's unterichoben? und ber Dr. Jeger hat eahner g'holf'n?! Red, Toni, red!

Toni (mindet fich und will fich bie Şände befreien). $D$ meh, Bater, o meh! la $\tilde{\beta}$ mich) aus. Drüct́ mich) net io, bu tujt mir io meh!

Fredinger (in fürchterlicher (Erregung). Reঠ, Ioni, red,

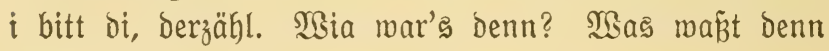
bavon?

Toni (burdy den Schmerz zur $\mathfrak{B} e r n u n f t$ gefommen). SBaS idf g'reot hab, ijt mahr, Bater, und mehr fann idf net jag'n. (3errend, um ihre Bselenfe aus den fie feithaltenden ફänden

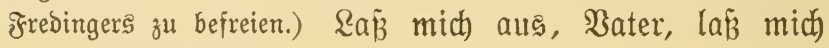
aus, ou tuit mir io weh!

Fredinger (hart). Net beoor'it mir derzäblt bajt, was d' von ber Iejtamentsunterichiaberei waj̈t und mia's du's erfahr'n bajt.

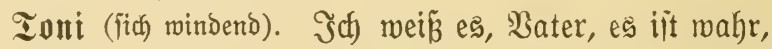
aber mehr barf id) net ag'n. $^{\prime}$.

Fredinger (feuctjend). Red. $\Im$ verrat di net, Ioni.

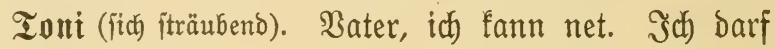
net mehr lag'n. $^{2}$ 
Fredinger. So wahr's an (Ssott im Seimmel gift, i verrat bi net, Ioni. Sag ma's.

Tont. J̈ch fann net, ich barf net.

Fredinger. Reb, Ioni! Mannit reb'it, Ioni, gib i dir a fectistaujend (sulden.

Tout (rubiger). Du verrat's midh net, Bater?

Fredinger. Ra, ficher net.

Toni. Schmör!

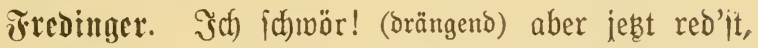
Toni.

Toni. Und bu gibit mir auch fechstaujend (s)ulden, wie ber Misia uno dem Schubiat.

Fredinger. S̃a, ou friagit's, aber du muaít ma alles derzähl'n.

Toni 2 llio .... (itoctt).

Fredinger (gejpannt). Med, red, Ioni!

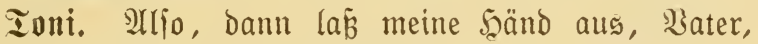
und bann werd ich dir's erzähl'n.

Fredinger (lǟst ihre Bielenfe auẹ). (Şuat, Ioni, i la oi aus, aber jekst red! MBia is das faliche Iejtament unteridhoben worben?

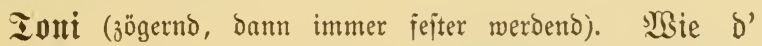
Mutter Iefitament g'macht hat, hat's net fo teitieren moll'n wie die Miß̧i und der ভchubiaf hab'n moll'n und wie Dann o' Mutter bas Ieitament g'macht hat, hat's ber Dr. Seger mit dem faliden veridbangiert uno hat der Miutter bas faliche Teitament zum unter= ichreiben geb'rt.

Fredinger (entietst). Der Dr. Seger! $\mathfrak{A}$ io a 
Sauner. Da war das Ieitament, was d' Mutter Ginterlaif'n hat, falich?!

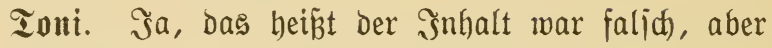
bie Unterichrift pon ber Mutter war echt.

Fredinger. Na fo a Banda! Dös fan ja ßer= brecher! Da hat d' Mutter ihr Scaus vielleicht gar mir vermacht g'habt.

Toni. Rein, bas nicht.

Frediuger. S̃a, marum ijt benn bann umtauicht mor'n? Mas ijt benn brin g'itanden?

Ioni. Dá̉ die Brüber foviel friegen müilien, wie wir, bie Miksi und ich.

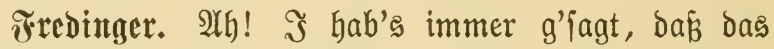

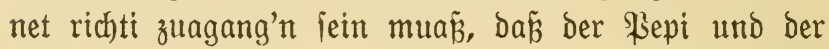
Raimund meniger friagt hab'n. Alber red, Ioni, mia fummt denn ber Dr. Seger da dazua?

Ioni. Ylber ber hat's ja mit'm Schubiaf abg'macht.

Fredinger. S hab's immer g'fagt, dấ er a Şader= lump is, der Rampeldiab, und i hab no immer Hedbt b'balten.

Iouti (befräftigend). Das ijt er audb) ein elender Serl, benn fonit hätt' er midh net auch jeb̧t betrügen mollen. Und bie Mibizi ...

Fredinger (unterbrectend). Mar die Miţ̧ a mit im Bandl?

Toni. Uno mie, Sater! Der Schubiaf und bie Miķi hab'n ja die ganze (S'ichicht anbandelt und mit Dem Dr. Seger ausfocht und weil ite's ohne mich net hab'n mad)'n tönnen, hab'n's mir's g'iagt.

Fredinger. So, du bait a mitg'halten? 
Toni. 2lber erij im lebeten Moment, Water! fannit.

Fredinger. Tas iagit jebt, weilit nimmer aus=

Toni (beteuerno). Nein, Bater, nein! Dá̉ das Iejtament umtauidht wirb, hab ich erit im lebten Moment erfahr'n, als nichts mehr bran zu ändern war, benn ber Dr. Seger hat's ichon fix und fertig mita'bracht g'habt.

Fredinger (entietst). Na fo was! was fo an Nobofat alles z'iammbringt, wann er amal zum rauben anfangt.

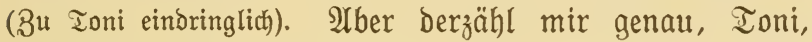
mia's eigentli hergang'n is!

Toni. SBie d' Mutter fo ichlecht beinand war, hat ber હchubial fortwähreno bei der Misi wegen bem Teitament benzt und hat g'iagt, fie foll mit mir red'n,

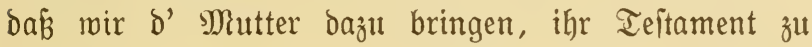
machen.

Fredinger. 2rber zu was denn? Mann's fan's g'mad)t hätt, hätt's ja a euch g'fört, benn $i$ und euer Mutter hab'n ja net in (S)ittergemeinid)aft g'lebt.

Tout. Unn g'bört hätt's ichon, aber bann hätt'n mir, bie Mabeln, weniger friegt, als wie bie Buabn, benn bann wär uns bas abgezogen morben, was wir vorausfriegt hab'n.

Fredinger (grinjend). De vierzigtaujend (Sulden, de d' Muatta auf's Szaus aufg'numa hat und euch geb'n hat.

Toni. Sa! Und bann mar's audh megen dir, Bater, weil ber Schubial immer g'iagt hat, wann fein Iejtament da ijt, mirit bu uns alles wegnehmen. 
Fredinger. So a SAauner! § wir meinen findern was megnehmen.

Toni. Und weil die Mibi und ihr Mann mir das alles immer vorg'iagt hab'n und mich immerfort fefiert hab'n, war ich auch babei und wir hab'n bann alles bereot.

Frredinger (unterbrechend). Mit'n Dr. Seger?

Iout. Nein; regen bem hat ber Schubiaf g'iagt, mit dem wirb er fichon iprectjen und ifn mitbringen.

Fredinger. SSlei mit'n falich'n Teitament?

Ioni. Stber nein, Bater, ba mar von einem falichen Teitament noch gar feine Red. Mir hab'n uns nur

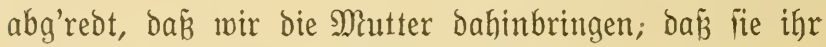
Teitament zu unieren (Siuniten mach'n ioll. Da $\tilde{B} .$.

Frredinger (einfarfend). Daß́ euch nichte a'zog'n wirb?

Ioni. Sa, Bater! Daß̧ une nichts abzogen miro

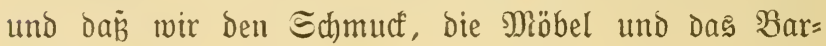
geld friegen.

Tredinger. $\Xi_{0}$, und da hat d' Mutter net moll'n?

Ioni. Aein! net io wie wir roll'n hab'n.

Fredinger. MBia Denn?

Ioni. Für ben $\mathfrak{I a g}$, an Dem die Mutter Teita= ment g'macht hat, haben wir uns abg'redt g'habt. Mir hab'n g'ichaut, daß̧ die Miki und ich allein blieben find.

Fredinger (ïct an bie Stirne greifend). Irum habt's a Den Raimund megg'ichicft?

Ioni. Sla ja, mo bie Dienitmäbln aud). Dann hab'n wir Der Matter wegen dem Iejtament zug'redt.

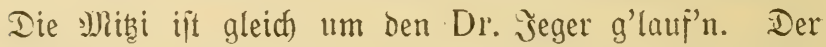
Schubiaf, ihr Mann, hat net weit von unierm şaus 
g'wart und da find's mit dem Dr. Jeger bald ba g'mefen und bas Ieftament ijt bann g'macht worben.

Tredinger. Mtber mia?

Toui. Die Mutter. hat nicht io mollen, wie's ber Schubial und die Mikỉ gern g'habt hätten, beim Şaus hat's ichon io viel Sponponaden g'mad)t und erit beim Sdimulf.

\section{Fredinger (geipannt). Und?}

Toni. Dann haben's halt auf alles, was bie Mutter wollen hat, ja g'iagt. Der Dr. Jeger hat alles niederg'ichrieb'n uno mie's zum Interid)reiben fommen is, hat er's fichell umtauid)t.

Frediuger. So a Banda! Uno du hait's g'mus̄t?

Toui (abmehrend). Tein, mir hat's ber Schubial erit g'jagt, wie fie grab vorm IImtauichen waren.

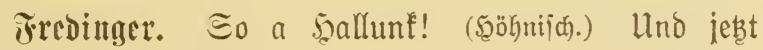
habt's do nix davon!

Ioni. Mer hätt' denn das denten fömnen, da $\tilde{B}$ io ausgeht! (Spöttijd.) Itber mehr als bie Buab'n hab'n wir boch.

Fredituger. Tös is mafr. Alber wia?

Ioni (höhnifa). M̉ie? Das erfahren fie nicht. SBir befommen a jed's jechitaujend Sulden extra und beim Íluggleich) fo wia bie Buab'n noch zweitaujeno, ba lyaben

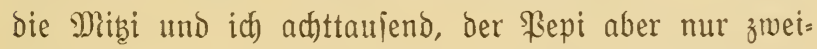
taujend und der Maimund gar mur eintaujend Ssulden.

Fredinger. Breitaujend Suldben. Denn für ben an Taujender muás $i$ ihn berhalt'n und den zweiten Taujender, den heb $i$ auf, bis er 24 Jahr alt wirb. 
Toni. Das ijt bem Raimund leine Sache. Er foll fich orum fümmern. Sch hab genug mit mir felber z' tun. Bei ber Selegenheit, Yater, möcht ich bich bitten um meine jechstauljend Siulden.

Fredinger. Schreib mir zma Beitätigungen, io wie's die Mitai uno der Schubiaf g'ichrieben hab'n.

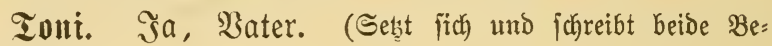
itätigungen.)

Frredinger (geft zur geöffneten Saffe, holt bas Beld und legt es vor Toni Gin). Da, Toni, fan bie fechstaulend (S)ulden und jest gib mir bie ganzen 3etteln.

Toni (gibt i็m ihre und bie zmei anderen ßeitätigungen). Da, Bater, find alle vier. Sch danf icjön, Sater.

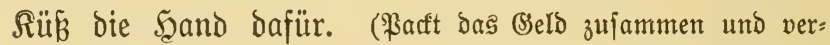
jorgt es.)

Fredinger. Du berfit nix davon jag'n, Denn junjt fommen bie Iotenvögel, ber Schubial uno die Miki, no amal und wollen no mehr.

Toni (beteuernd). Selbjtoeritändlich), Bater. Sch hab genug von ifnen, bu fannit bich auf mich verlafien. Utber, Bater, bu barffit mich auch nicht verraten, bu hait g'id)wor'n.

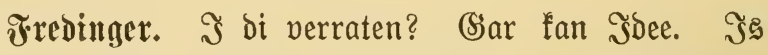
eh \{cho z' ipät. Wann i bös por aner Biertelitund g'muß̄st bätt, aber fo. (马u Ioni). $\Im$ wir di net ver= raten und bu derfit mi net verraten und berfit bir a nix bei ber Mib̧i uno ihr' $\mathfrak{n}$ Mann merfen laffen. Muá̧̆t mad'n, als ob gar nix g'mejt wär. Beritehit? Denn er is a elendiger Şaberlump und fie a grofe Ranalli. 
Tuni. (Sanz natürlich), Water, benn jonji hätten

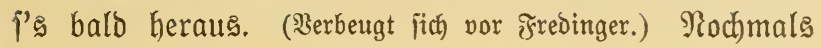
banfe ichön, Sater, fün bie Şand! (Will gehen.)

Fredinger. Wo willit denn hin? Rannit glei ba= bleib'n. (Sieht auf die Mhr.) S' müifien eh alle bald ba jein!

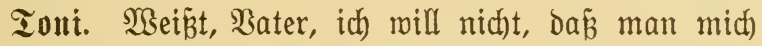
bei bir allein fieht. $\Im$ dh fomme gleich wieder.

Fredinger (mit dem Ropfe nidend), (Šuat is! Syeut= zutag muá ma recht vorfichtig jein. (Seh ba burchs Bureau auß̉i und wann man bi fieht, bann fag nur, bu hajt mur nachg'ichaut, ob d' IYndern icho da lein! (Ģeft mit ihr bis zur Bureautüre, blicft zuerit finaus und jagt leife): Die Ruft is rein. Die Schubiafleut fan icho meg. fannit idjo geh'n. Alber fomm glei wieder, Ioni!

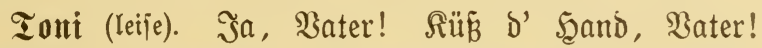

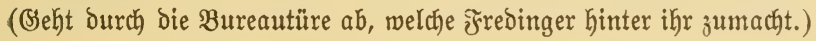

\section{Dritte Szene. \\ Fredinger allein.}

Frredinger (von ber Türe zurüđffommeno, zum Schreibtiīch

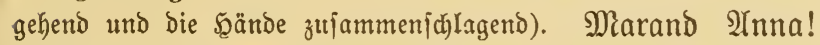
is dös a verlotterte Banda. So a (S'findl. So haut= (đ)lect)te Reut! Führ'n mit einander a (Saunerei um b' andere auf, nur um andere zu betrüg'n und bann betrüg'n iie it gegenjeitig a no. So a elendiges Flumpert, da is ja unj'rans an Engerl bagegen uno ma is do urbentli g'rajt in ber $\mathfrak{B e l t}$ und hat ficho a 
paar iđjöne Stücfl'n außaga'itecft, be fi anichau'n laij'n finna. suber fo was! Na fo was! 3'erit halten's z'iamm, bamit's mei veritorbene Frau zu an für eahner günitigen Teftament bring'n. Mria das net geht uno bamit haben's icho g'rechnet, unterichiaben's a falich Teitaneut uno ber Dr. Seger hilft eahner dazua. '̌s a an ausg'fuchter Şaberlump! z'erīt hilft er eahna burten und jeşt laşt er fi pon mir a fauf'n. Uno warum tuan's Das alles? Nar wegen Dem drectigen (Sjeld uno

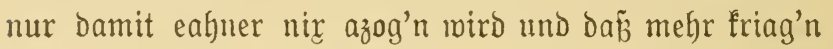
roia die Buab'n. Unto no daz̆la jeb̧t mährend dem Srozez hah'n fie it immer an mi zuchiorucft mia o' un= (c)uldigen Samperlı und haben ma furtmähreno vor= g'jammert, dá̉ net ie, iondern ber Dr. Seger und bie

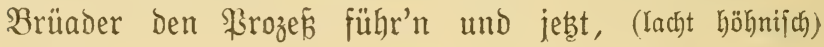
mann's net fo traurig mär, mïtẩt ma lach'n, jeşten machen's miteinander wieder aus, daís eahnare Brüaber abiorah'n. Saîfen fi von mir zahl'n, um den 距epi uno

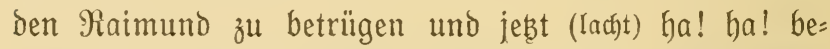
trüg'n fie fi gegenfeitig a no. Uno das woll'n (S)'id)mijter jein, de z'iammbalt'n. Dös is a Rumpenbando, an elenbige! Te Mabeln ian Ranallina, wia's fane ärgeren gibt uno ber Schubial, mei Schwiegerjuhn, is aner ber

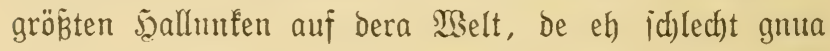
is. Ier 5aberlump is net umionit oon ber unter'n Sirenz fumma, Dort fan bie gebor'nen Rauber her. Da is ma ja im Ranbesgericht unter lauter Sträfling fich'rer, denn da rẩ ma menigitens, wo ma is und mit wem man's z' tun hat, aber da? (Epuct aus.) Rfiui Ieufel!

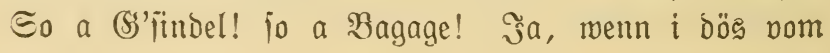


Tejtament nur um a Biertelïtuno früher derfahr'ı hätt, nur um a Biertelitund! vielleidbt mär's gar mit zehu Minuten ganga, bann wär all's anders wur'n und i bätt mir das ganze S'eld bajpar'n finna. (ভct)merzlitf.) Mei liab's, mei ichön's, mei ehrlich perdient's (Seld! Itber io! Jeb̧t is pfutich! WBas mukert's mir, wann $i$ jest an Sramall anfangert? Was Denn? (Sar nix! Denn im (S)uat'n gibt das filumpert bös (S)eld nimmer= mehr her und wann i's auf a g'ridutlidje Âtustragung anfumma ließsert, fo wurben's eing'naht. Mer hätt

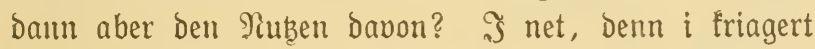
das Şaus höchitens, wann's guat geht, mur mieder mit

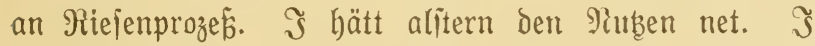

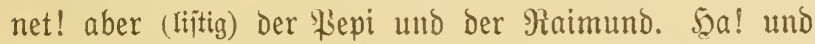
bevor i's denen laijert, Dem $\mathfrak{B}$ epi no eher, wann er's a perwirert, aber dem Yiaimuno, (aufiareiend) na! . . na! ... bevor i's dem laifert, bewor foll's lriag'n wer will und wann's de (Saunerbanda von mein Schwieger: fuhn, dem Sgaberlumpen und de zwa Ranallina von meine Madeln is, aber dem Rausbub'n, Dem Raimuno, (wild) na! na!... um nix in ber Melt tund wann Der Seerrgott jelber aberiteigert uno ihm helf'n tät. Unter gar faner Bedingung, (verbififen) benn i mag'n net! I will'n net! Er is mir jo z'mider. (Wütend.) Umbring'n funnt i den Buab'n, den ... (itok̃meife zwifhen Den Bähnen hervorziffhend) Den Strold)! und hin mer'n will er a net, ber Şund! (Rebet fict inmer tiejer in bie \$erbiffenteit binein.) (5r is a nix anders als a Baitard! Die Cilli hat ganz recht, mann's immer lagt, er is net von mir, umionit fann er fi net vertrag'n 
mit ifr, denn er is ganz anders wia meine andern Rinder. Und was er ii nur einbildt? $\mathfrak{M a s}$ er nur glaubt?! Эs nix, hat nix, lebt nur vom Szunger ober

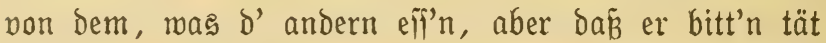
ober an zuared' $n$, oder mann er menigitens was tät,

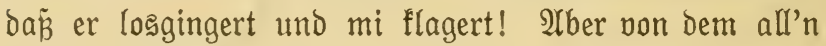
gar fa (S)'ipur. Saß̧t fi bei mir überbaupt net an= ichau'n und mann icfo, fo itreit er mit mir ober er rest nir uno fajaut mi an, ichaut mi an ... i was net mia ... grad als ob er mir lag'n mollt (itorft)...

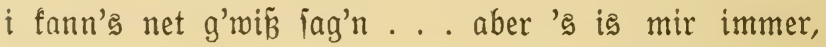
i maßs net mia... i hab das (s)'fühl, als ob ... als ob er mir über wär, als ob er mi durchichau'n tät uno bá̉ er mi nur fhonen tät, weil $i$. . meil

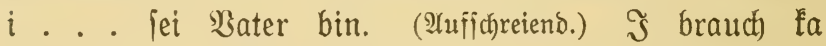
Schonung, i will la Schonung, won eahm icho gar net! Denn $i$ mag'n net, er is mir io verhaf́t, dẩ $i$ ifn er= würg'n fönnt, wann er mir nur an Urjach bazu gebert, da jein ma meine andern findern, fo nix nub als fan, viel liaber. Mein Schmiegeriuhn is an elendiger Scader= lump, meine Mabeln jan leichtfinnig, woll'n nix arbeit'n, preif'n ma 's (seld a, fan bumboll હchulden und babei

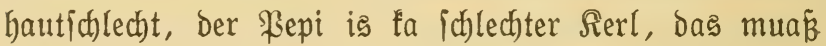
i lag'n, aber leichtinnnig bis in bie Sinochen, oraht, lumpt und fauft, aber alle fan's ma immer no viel liaber als Der Rausbua! (Sein Blicf fällt auf bie auf dem Schreibtifa) liegenden Bettel, er jtofft, finnt einen Moment nach und nimmt Dann bie vier 3ettel vom Schreibtijø.) Jeb̧t hätt $i$ megen bem ¿ausbuab'n balo auf bie Bejtätigungen vergeffen. Sest muá̉ i's aber glei einjperr'n, benn bei jolche Toten= 
pögel, mie mei Sdmiegeriuhn und die MadIn fan, muañ ma fi g'hörig hüaten. Ie fan imitand, wann ite fich brauş'n treffen, reden fie fich mitanander $a b$, fummen g'ruct und ftehl'n ma bie Betteln aus der Srand. (Die

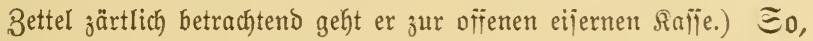
fummts nur, jebt wero ma euch auf Nummer Sicher bring'n, in mei eijerne eini. ( nur ichön liegen bleiben. (Sabließ̄t bie Saffe und probiert, ob fie gut zu ijt.) So, jebst fönnen's icho fommen, mei מ̃err Schiviegerjufn und meine liab'n Maderln! Mann's a grof̈e (Einbrect)er lan, ba kummen's do net eini. (Sieb=

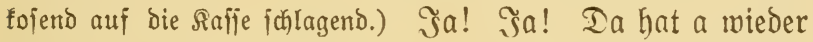
amal 'S Rücferl g'icheiter jein woll'n, wia d' Senn und hab'n glaubt, mi, ben alt'n Fredinger, finnen's abibrah'n! Utberbaba! (Madjt, ben Daumen zwifdnen Beiges und Mittelfinger itectend, eine reige.) Dö hab i jeşt alle oree in ber Szand und

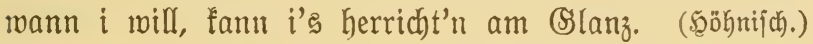
Der \&ampeldiab hat glaubt, er hat's g'icheit ang'fangt mit bie żma Bejtätigungen, aber jo hängen's do, denn De zwa Bettelı fan von alle zwa unterichrieb'n, hab'n basfelbe Datum, i glaub net, dás fi bald aner find,

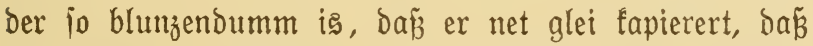
be lectstaulend (suldoen extra nur friagt hab'n, damit's

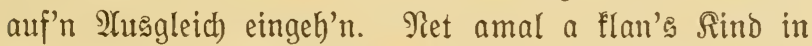
der Miag'n!? Ter Schubiaf̂, der hat mirfli glaubt, i gib eahm's Sseld obne de Bejtätigungen. $\Im$ bin do net erit heunt auf d' SBelt fumma, damit $i$ mi von io

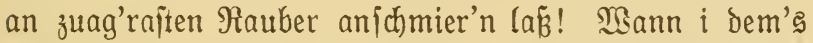
(S)eld ohne de zrwa Bejtätigungen gib, jo gengan's anfach auf ben $\mathfrak{A}$ usgleich net ein und i fann's no amal zabl'n. 
Pa, fo g'icheit fan mir a no, Buricherl! Dö foll'n ma no amal Manderin mach'u, bann wir i eafna mas

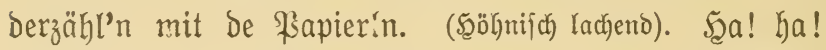
mar'n grad net billig und i bab meg'n ber Ioni melyr zahl'n müafi'n als was i glaubt hab, aber besmeg'n mach i do a ganz a guat's (s')ichäft. Der ßrozen hat fi rentiert. (马ähleno.) Die Mibi und ifgr Mann, oer Schubiaf, hab'n z'iamm jechstaujend (Suldon friegt uno die Toni a fechstauieno, macht zmölftaujeno. Iazu fommen de zimeitaulend, be i beim 2 tusgleich auszahl'n

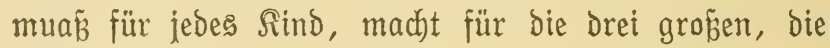
Mibi, bie Toni und ber \$epi mitanander iechstaulend, aljern mit de zmölftaufend z'iamm achtzehntaujend (Sulden. Der Maimund friegt mur an Iaujender und ben erjt beim 24. Sahr, bis bahin hat's no lang 3eit; ben zmeit'n Iaujender behalt $i$ mir als (Erziehungsbeitrag, Denn $i$ muaş'n derhalt'n bis er grofjiährig is und wann er mi net mehr fojt, als wie bis jebt, deripar $i$ mir den Iaufender a, Denn jeit jei Mutter tot is, hat ber von mir no fan firenzer g'feh'n. Sia, i wir fido (d)au'n, dás i a jeşt net j’ furz fomm! Das mär'n aljo im (sanz'n adytzehntaufend (sultoen, bazua fomm'n die Dofteripejen und mas i dem Dr. Seger zahl'n muaß́, dañ er rufig is, uno was i mein Dofter, bem Dr. Şatichef extra zahl'n muaj̃, (iich) unterbrechend) den Scatichef, den muás i ordentli zahl'n, benn ber hat fi's verbient. Mird mi vielleicht alles in allem auf a vierzigtaujend Sulden fumma. Na uno was frieg i bafür? '马 Şaus! das ijt auf a zmeihundertfünfzigtaujend (S)ulden g'ichäbt, da fummen de vierzigtaujend weg, de mei Frau 
für d' Madoeln aufg'numma hat, bleiben a żmeifhundert= zefntaujend, dann bie vierzigtaujeno, de i zafl'n muá̃, bleib'n mir rein hundertitiebzigtaujent (Słulden, (Iäct)elnd) bas is do a guat's (S')ichäft, no bajı mo's salts, unter uns g'iagt, wirfli meiner Frau und net mir g'bört hat. atber dös geft niemanden was an, denn heilig ifit bas Erigentum, wann's mir g'hört. $\mathfrak{F}$ tann f'frieb'n jein, wann nur alles guat auslauft beim 2lusgleich)! (尔ect.) Warum ioll's net guat ausgeh'n? Es muaí guat aus= geh'n, denn $i$ hab eh idjon alle im sacf. Der Schubiaf, ber ftimmt net $a b$, reot aber am meiften brein, bie Mibi uno bie Ioni fan icho auf meiner Seiten, ber \$iepi lagt a ja, weil ihm nir anders übrig bleibt und ber Raimuno, ber \&ausbua, hat nir z' reden, weil er no minorenn is. Wann de andern ja jag'n, fann er lato gar nir tuan und muab́s's, ob er will ober net, abi=

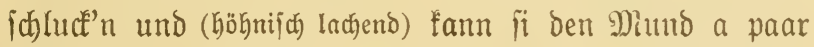

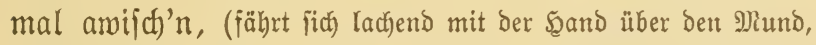

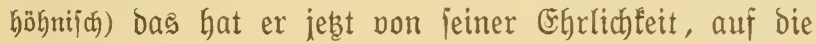
er fi fo viel einbild't, Der bumme fierl! Uno wann er no fo ehrlich uno anitändig is, nuken tuat's ifym do

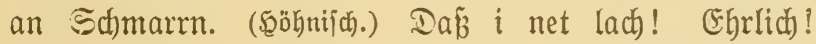
Înitändig! 3' bumm is er bazu! 3' Dumm! Denn ehrlich fann nur a bummer Sierl fei! Denn a g'icheiter Serl maß̉ mit jeiner (Ébrlichfeit mas anders anz'fang'n, als ria jo bumm z' jein und ebrlich z' jein. Nats ob er ras bavon hätt obar eahm mas tragert? Ier joll jeine ভdymejtern uno fein Sdywagern, den Schubiaf, anidhau'n, Denen tragt's mehr als eahm. (Es flopit an ber mittleren $\mathfrak{x}$ üre.) Mit feiner tepperten Eyrlichfeit!

(৫ே 
flopit wiederholt.) Der Irottel. Drum jag $i$ ja. Effrlich fann a jeder bumme ferl fein. (Die Türe öffnet jich und Gerein tritt Dr. Şatichef und bleibt einen Moment ruhig zuförend iteḥ'n.) Denn ehrlich mährt nur bann am längiten...

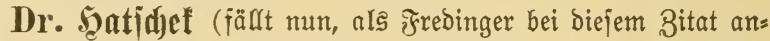
gelangt ijt, mit folgenden ergänzenden $\mathfrak{B S}_{0}$ orten ein) mann es am menigiten abgenübst miro.

\section{Bierte Szene.}

\section{Frebinger und Dr. Sgatidef.}

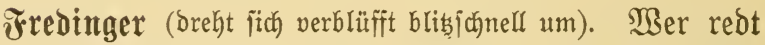
Denn da? (2lls er Dr. ફatichef bemerft.) IYh, Der Şerr Doftor!

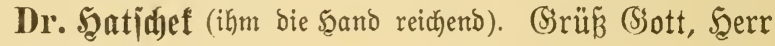
von Fredinger!

Fredinger. Şab die Ehyre, Saerr Dofftor! (5at fith inzwijchen gefaj̄t und um zu wiffen, wie weit Dr. Satichef jein Selbjtgeipräch mit angehjört, Den Biedermann ipielend). Net mahr, Scerr Doftor, $i$ hab rectst, wann $i$ iag, es is idwer auf bera Melt für an ehrlichen Menich'n, beun bie Menichen fan fo viel ichlecht?!

Dr. Şatidget. Da itimme ich bollfommen bei, benn felbit bie ehrlichen Menichen find fo nerborben, daß einem vor beren Ebrlichleit oft fhlecht merben fann.

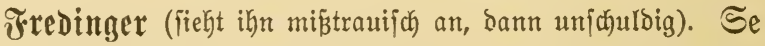
hab'n mi ïberraicht, Seerr Doftor! § hab Shna gar net fumma g'hört und bin ordentli berjarocfen, mia S' plöblich mia a Seift bag'îtanden jan. 
Dr. Şatiadjef (lächelnb). Das bachte ich mir, şerr von Fredinger, benn idh habe zmeimal angeflopft, ofne eine SIntwort zu erbalten und da habe idf bie Iüre geöffnet, um mich zu überzeugen, ob Sie herinnen find, Şerr von Frebinger. (5s warten nämlich ichon alle brauß̄en.

Fredinger (Fich entijuuldigend, bäflich). Bitte, bitte, Şerr

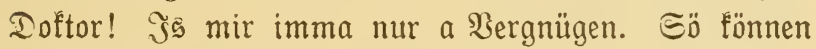
bei mir einafumma, wann $\Xi^{\prime}$ moll'n. Sö fan bei mir

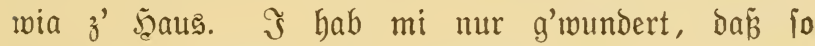
momentan jemand hinter mir iteht uno redt. 2 ber Şerr Doftor, Sö jag'n, es wart'n idjo alle?

Dr. Şatidjef. Jaa, alle, fomeit id) gejehen habe. (Şertraulich zu ふ̌resinger.) Sit alles in Dronung? Şaben Sie mit Эgren Rilldern...

Fredingcr (unterbrectend). Allles, alles, serr Doftor,

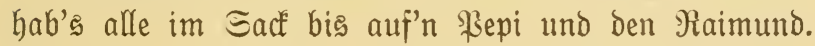
Der ßepi wiro fane Annitäno madh'n uno mit'm Piaimuno ...

Wr. Saatidjet (einfallend) werden wir idjon fertig merden.

Fredinger. Allitern, dann fann's angeh'n!

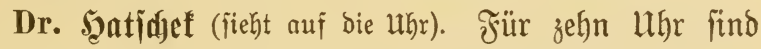
alle herbejtellt. Es fehlen jmei Minuten. (ङs flopft.) Das werden fie fein.

Fredinger (idnerl). $\Im$ glaub a, 5err Doftor! Allitern

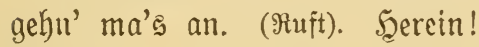




\section{$-124-$ \\ Fünfte $\subseteq_{z}$ ene.}

Frebinger, Dr. Şatidef, Schubiaf, Mritzi, Ioni, ßepi

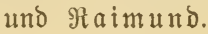

(Die Iüre öffnet fïh) und es treten ein Schubiaf, Mititi und Ioni, zum Schluffe Ginter ben anderen \$epi und Raimund.)

Sdubtaf. SSuten Iag, Sdbiegerpapa! Suten Iag, Sherr Doftor!

Mitji und Toui. Rüßß b' Şand, Bater! (Suten Iag, Şerr Ioftor!

(ærebinger und Dr. Şatjøef danfen.)

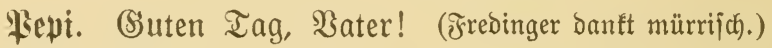
Şabe bie Erbre, Seerr Doftor! (Doftor Danft.)

Maimund (Gäflich). (Siuten Iag, Bater! Sabe bie (5hre, Sherr Doftor! (Doftor Danft.)

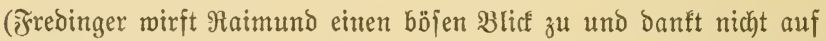
defifen (3rußs. Macht eine abmefrende, wegmerfende Bemegung mit

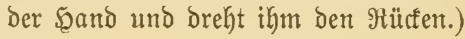

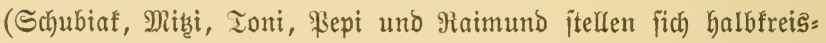

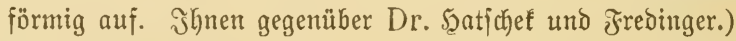

Dr. Seatidyet. So viel idi lebe, find alle (erb= berechtigten hier perfammelt. Sidfst wahr?

:Ille. ร̌a!

Dr. Şatid)t. Sun, bann fönnen wir beginnen. (Entrimmt feiner 2lftentajche ßapiere, legt bie Iajage wieber nieber unb bie \$apiere in ber Şand haltent, fpricht er, teills frei,

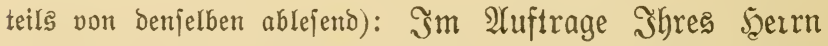
Baters habe id Shnen folgende Mitteilungen zu machen: (mit erfjöhter Stimme und Betonung) Sbzmar ber günfitige 2lusgang des \$rozefles für Şhren şerrn Bater zmeifel= 


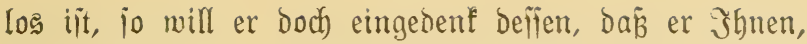
feinen Sindern gegenüber, immer ein guter ßater war...

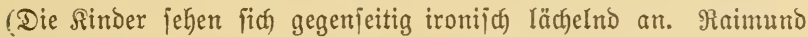

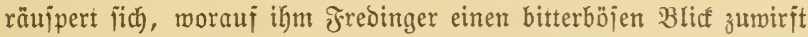

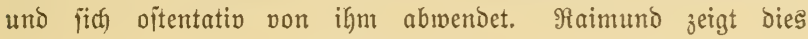
läcţelnd ærepi.)

Dr. Syatidyef (hat eritaunt Den Sopj erhoben, jein Bliff gleitet

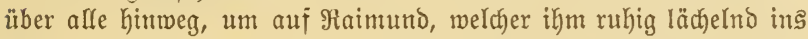

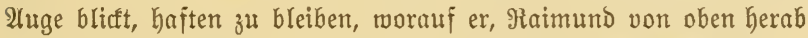
anjehend, mit erhöhter provozierender Betonung gegen denjerben ge=

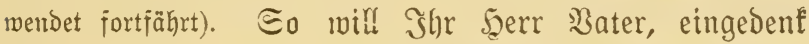

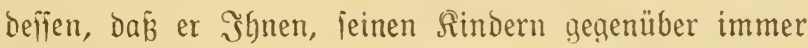
ein guter Bater war uno aud immer lein wirb, den zwiichen Şnen und ihm ichmebenden Frozé aus ser

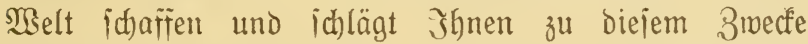
folgenden auB̈ergeridhtlichen Bergleidh por. Sie, bie

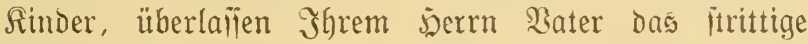
Dbjeft, das heiß̄t, bas pon Shrer Mutter ererbte 5̃aus,

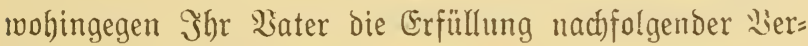
prflidhtungen aus freiem willlen übernimmt. Eritens bie 3ezahlung ber bisher beiderieits aufgelaufenen Spejen, zmeitens erfält jedes der grobiäbrigen finder bie Summe von zmeitaujeno (s)ulden bar ausbezahlt. (Sich)

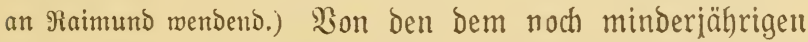
Seerr? Haimund zufommenden zreitaujend (S)ulden be=

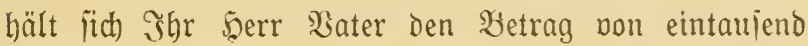
(sulden als Erziehungsbeitrag zur itandesgemäß̄en Er= haltung zurïct. Die rejtlichen eintauiend Sulden merben bem 5errn Raimuno nad) Erreichung feiner (5irof $=$ jäbrigfeit ausbezahlt uno bleiben bis Dorthin in 2 er= waltung Э̧res Şerrn ßaters. (IIn alle gemendet.) (5e 
bleibt jomit Э̧hnen überlajien, biejen 2̂tuggleich) anzu= nehmen oder benjelben abzulehnen.

\section{Sdubiaf. Wir nehmen ihn an.}

Mtaimund (voripringend, erregt). Sitber ich nehme ifn nicht an. S(ch) nicht. Unter feiner Bebingung. Э(h) muß bas befommen, was abgemadit mar.

Dr. Szntiducf. Das befommen Sie ohnedies. $\mathfrak{U}$ b= gemacht ift aber nichtes worden.

Minimunt. D ja, Seerr Doftor! Dr. Seger hat mir

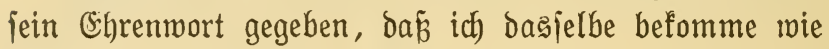
bie andern, zmeitaujend Ssulden.

Dr. Sgatidjef (abwehrend). Das ift wohl möglich, aber

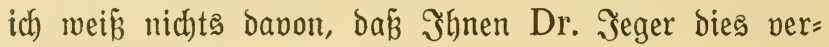
iprochen hat.

Naimund (beteuernd). Эa boch! Doch, Seerr Doftor! uno fein Efhrenmort hat er mir gegeben, baj id bas Sanze betomme.

\section{Dr. Satidgef. Das befommen Sie auch!}

Maimnnd. Das ift nidht twahr, id befomme weniger!

Dr. Saatidef. Im übrigen fommt es auf Sie allein nicht an. (5s frägt fich, ob Şhre älteren großjiährigen (Se) fhrifter einveritanden find.

Fredoinger (freç). Şanz natürli! ( $3_{\mathfrak{u}}$ Den älteren Rinbern gewendet.) Allfo nefmt's $\Im \mathfrak{h r}$ 's an?

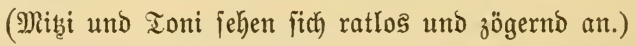

Pepi. $\Im$ begreif wirfli net, warum ber Maimund meniger frieg'n joll.

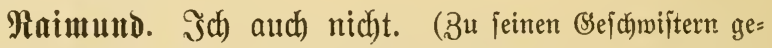
mendet.) Эgr bürft's nicht annebmen!

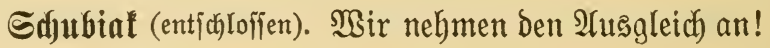




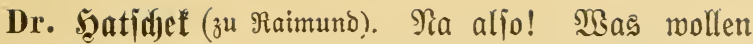
Sie benn meiter?!

Maimuth (zornig zu Schubiaf). SMer hat denn bich gefragt? Scajt bu benn ba mas zu reden?

\section{Saubiat. Mefhr wie bu!}

Maimund. Das merden wir erit fehen! Saajt denn du geerbt? Deine Frau hat geerbt und bie Ioni, ber Sepi und ich. Menn's nach Recht ginge, folltejt bu eigentlich gar nicht hier fein. (3u Mithi und Toni). YUlfo Mibi und bu, Ioni, ihr fagt's nein! Shr werdet euren jüngiten Bruber nicht ichäbigen.

Pepi. Recht haît, Raimuno, ou muñt bas gleiche frieg'n.

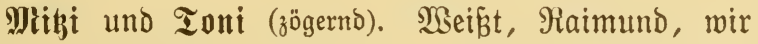
fönnen net anders.

Fredinger. Na alio, ijt fchon angenommen.

Maintund. Sït nicht mafyr! Das gibt's nicht. (Mird zu feinen Sdymeitern.) 'raas! Shr habt's den Mut, das anzunehmen? $\Im \mathfrak{h r}$ liefert's euren jüngeren $\mathfrak{B r u d e r , ~ u m ~}$ ben ihr euch annehmen folltet's, ans Mefier? Schämt euch, nur jo auf ben eigenen Borteil bedacht zu jein!

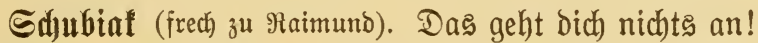

Maimutd (zu Sajubiaf). Dich hat überbaupt niemand gefragt. (Zu Dr. Şatichef.) Scerr Doftor, Der Mann (auf S(j)ubiaf zeigeno) hat überhaupt nicht bie Berechtigung, hier zu fein!

J). Şatidjef (in Der Rlemme, langiant). S̃a, Şerr

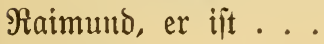

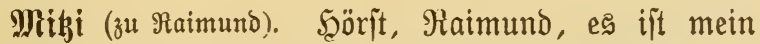
Mann! 


\section{Srfutbiat. So eine Rectfheit!}

\section{Frrediuger (iefundierend). (Ssanz natürli!}

Miaimund (auj ভchubiaf losfahrent). M̉as, Rectheit?! Das verbitt ich mir, jonjt erlebit was. Wer iit benn frecher mie du?! Sajt bu benn je etwas anderes als 3wieipalt geichaffen? Red nichts brein. Das geht bich nichts an. Du verfaufït immer bie Şaut von einem andern und jest ift's meine saut uno $u$ m bie muś ich mich rebren.

Plepi (zu Schubiaf). Sei ftad, Der Raimund hat recht. (3u Maimuno.) Du muñt dei Sach'n grad fo wie wir frieg'n, funiten fag i a na!

Inr. Jeatidnet. Her er befommt ja aud dasfelbe.

Matmund. Das ift nicht wahr, Scerr Ioftor. Sie haben es felbit vorgelejen. Bei meinem 24. Rebensjahr taujend (Suldoen uno taujend (suldoen bebält fïch ber Bater zurïcf́.

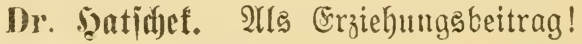

Tredoinger. S⿱

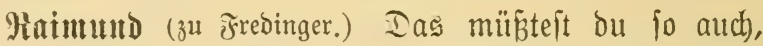
aber bu tuit es nicht.

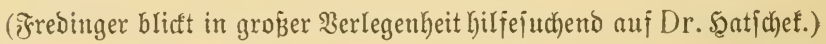

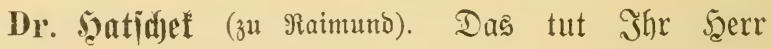
Bater ohnedies!

Minimund. 2lber wie!? MBenu er es io meiter= machen wirb, wie bisher, iteft er ben Taujender jamt Den Zinfen ein.

Fredinger. So a Frechbeit!

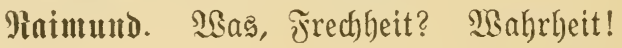


1)r. Şntidjet. Das geht zu meit, Jeerr Raimund! Эhr Şerr $\mathfrak{B a t e r}$ wird fich feiner \$flichten Şhen gegen= über immer bemuät bleiben.

Rainumd (G̈̈hnifh). So wie er fich derielben bisher bemuñt geblieben ift und mir nichts gegeben hat!

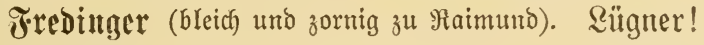

Raimund (fährt auf Fresinger los). Mer Rügner?! Mas Rïgner?!

Mitji und Toui (wollen ærieden fitiften, zu Raimund). Atber Raimunt, geh ichau ...

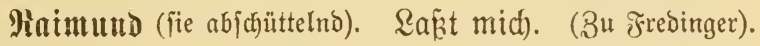
Saajt bu mir mas gegeben, feitbem bie Mutter tot ijt? Saajt bu midh nidbt hinausgemorfen auf bie Straf́e,

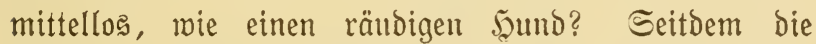
Mutter bie Alugen geidhlofien hat, habe idf von bir nicht einen seller erhalten. Sm (Ssegenteil, berfolgt hait bu mich, wie und wo bu nur fönnen hajt!

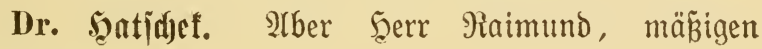
Sie fich!

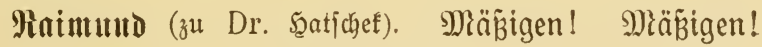
Seerr Doftor, das ijt leicht gejagt.

Fredinger (bazwifden rufend). Das ift alles net wahr, was er fagt. Err liagt.

Maiuund. Mas ijt nicht wahr? Mas? Scaft bu mich etwa erfalten ober hab idh oon bir auch nur einen roten Şeller erhalten?

Fredinger (verlegen, wiro wild und frech). Du hait immer friegt, was o' braucht hajt.

Raimund (aufichreiend, mit bejdwörend Gimmelwärts ge= hobener 5̧and). So wahr es einen (Sott im şimmel gibt,

s a lm, Ein eb̆rlides altę ફুaus. 
Das ift nicht wahr! ( $3 \mathfrak{u}$ feitten (3ejchwiftern gemendet.) Miķi! Ioni! 'Sepi! habt's ifr nicht immer gefört und gejehen,

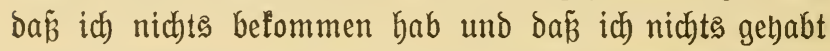
hab? (Ehrlich) jagt's es. (Miţi und Ioni winden und drehen fich verlegen.) Secraus bamit! Rüg ich) oder nicht? (2uts

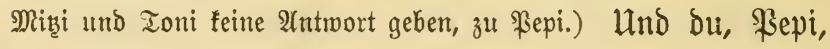
bu wirft...

Fredinger (unterbredjend zu ßepi!

Maimund. Er foll nur jagen, mas mahr ...

Pepi (ichnell). So viel $i$ wañ, haît nia nix friagt, benn ou hajt oft net amal z'effen g'babt, vom andern net z' reben.

Maiumund. Danf fajön, Fepi! Du bijt noch ein efrrlidjer Bruber.

Fredinger. Der dir z' lieb reot.

Maimutud. Rein, ber aber menigitens nod ein Scerz für feinen Bruber hat, nicht fo wie ifr, (mendet jich zu Miţi und Ioni, veradgtungşoolf) bie ihr eud nicht traut, Denn ihr fönntet's euch's verberben.

Mithi und Toui (zögerno). MSir wiffen's ja net!

Raimund (empört). Shr wipist es nicht? Da hört fich boch alles auf. Shr, bie ifr's rmal beredt habt,

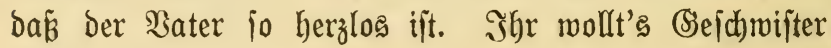
fein? Э̧hr, bie ihr bie älteren jeid und für euren

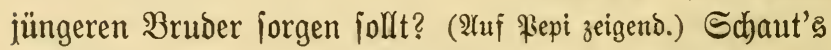
den Bepi an, der ijt menigitens noch ein Bruder, ber, wann er auch felber nidyts bat, aber boch noch ein \$cerz in ber Bruit hat. Sidht fo wie ifgr, (will vor fie hin= 
treteno) benn ihr feio mur faliche, herglofe Egoijten! Bfut ber Teufel! (Spuct aus vor innen.) \$Ffui der Teufel!

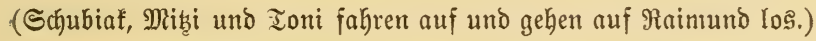

Sdjubiaf. Frecheit!

Mitiji. So a fedfer Ferl!

Toni. $\mathfrak{2}$ usppucten tut er por uns!

Raimund (ipringt mit geballter Faujt auf Schubiaf zu). Menn du nicht bas Maul hälï und mich beleibigit, fdlage ich bir bie 3ähne in ben Rachen.

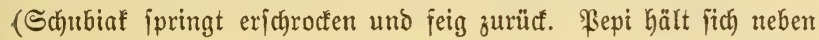
Raimund.)

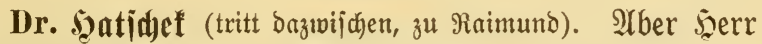

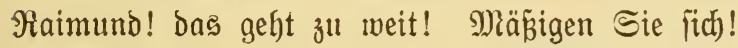

Maimutmo (iid) von Schubiaf abmendend zu Dr. Satichef wild). Määigen? Sie fommen immer mit dem mäßigen, ftatt daß̉ Sie einen armen um jein Red)t fämpfenden Menichen unteritïzen. Mäßjigen Sie fich, Şerr Doftor, menn Э̧hnen bie Şaut über bie Dhren gezogen twarben foll. Und fo wahr es einen (Sott im Simmel gibt, ber $3 a t e r$ lïgt! Seitoem bie Miutter tot ift, habe ich nicht einen Şeller befommen.

Fredinger (einfallend). Das is net wahr. I hab ihn anftänbig derbalten. Er liagt, ber Rausbua, ber Berleumber!

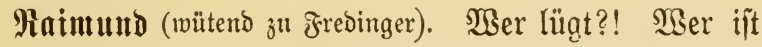

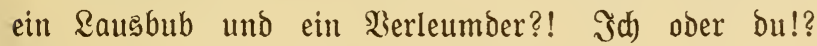
Эd) hab noch niemandent um fein Erbteil bringen

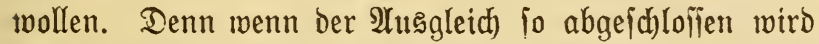
uno du mich) fo mie bisher erbältît, gibjt du mir mieder nidbts und itecfit ben Taujender ein. 


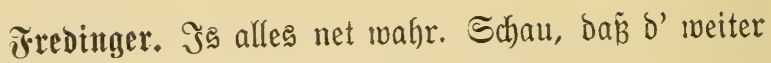
fommit!

Maimund (wirl, mit brohent zum Simmel emporgehobener Şand). Und wenn biejer âsgleid zuitande fommt, io ijt er ein an mir vollführter Betrug!

(ङrebinger hebt bie Şand, Schubiaf ebenfalls; es bringen alle auf Raimuno ein.)

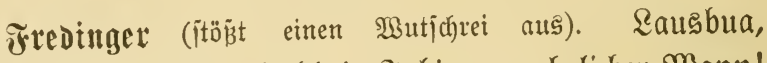
elendiger! Şinaus mit bir! $\Im$ bin an ehrlicher Mann!

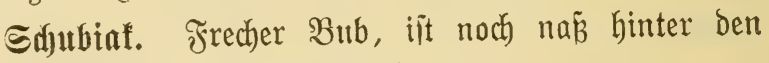
Dhren. (Dringt auf Raimund ein.)

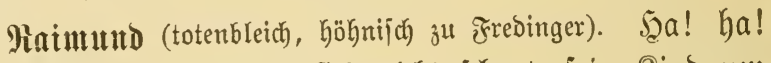
ein efrlicher Mann, ber fich nicht icheut, fein find um fein mütterliches (srbteil zu betrügen.

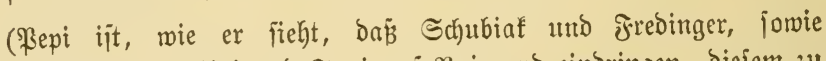

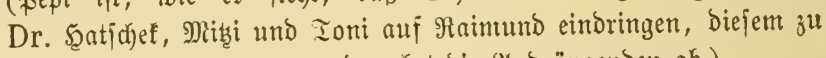

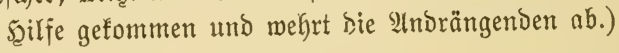

Fredinger (mütend 孔u Raimund). Şinaus mit bir, Rausbua frecher! Schau, bañ d' weiter fommit, fonjt g'ichiect)t no an Unglürf́.

Miaimund. Das werden wir erit nod fehen.

Edubiaf. Sinfs und rechts foll man bem Saus= bub'n (will von rüctwärts auf अaimun joblagen).

'Hept (reist ifm bie 5̧and herunter). 2 , bös gibt's net,

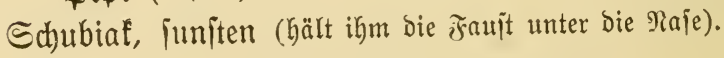

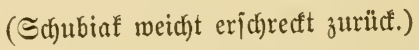

Mlitji (pactit ihren Mann beim $2(r m$ und zerrt ifn). SCh bitt bich, Schubiaf, mijh bich net eini. Sonjt friegit

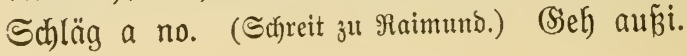




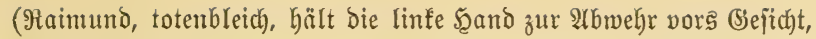
mit ber zur ₹aujt geballten red)ten ફ̧nnd holt er zum Schlage aus. \$epi, immer ifm zur Seite, hält bie Raimund gegen bie Türe brängenden ßerfonen Fredinger, Dr. Şatjhef und Schubiaf zurüdf.)

Mainnutd (mit zornbebender Stimme). Der erĩte, der mich anrïlyrt, dem Sinade (Siott!

Bepi (itellft jïc) vor Raimumb, den 2trorängenden zurujenঠ). 3'rucḱ, jag i, z'ructé, junjt ...

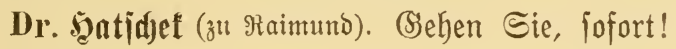

Maimutro. Das ift meine Sache.

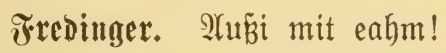

Maimunto. Sch bin ja froh, wenn ich brauben bin,

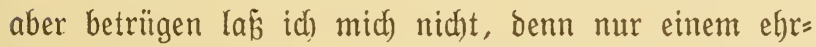
lidjen Mieniden fann fo was paifieren, wenn er hinein= fommt in fo ein gottwerbammtes, vermaledeites Şaus!

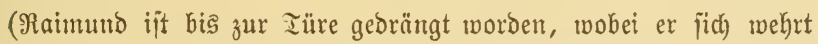

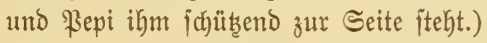

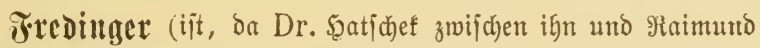

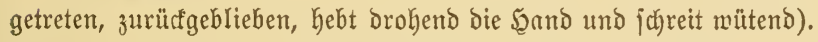
Rump! Rausbua! (Ehrabichneider! auß̄i mit eahm!

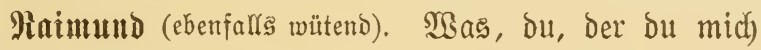
betrügen willit!

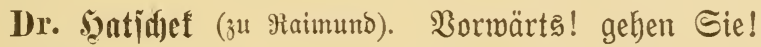
gehen Sie!

Fredinger Schau, dẩ d' weiter fummit. Du bijt a (Ehrabidnneider, Der uns ebrlichen Leut'n ó Eytr $a b=$ (d) neib't. 


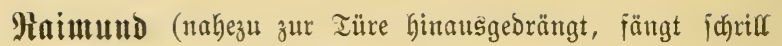

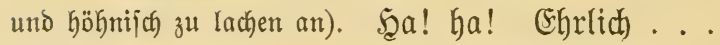

Fredinger (unterbridgt ifn). Suld , Şund! und mert

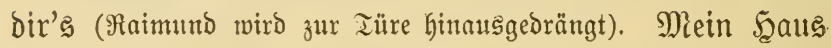
is an ehrliches, an altes Şaus. (Die Türe fällt Ginter

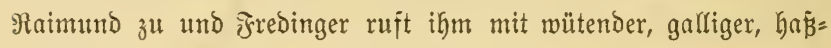
erfürlter Stimme nach.) D Du Bajtard!

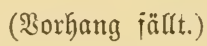

Ende Des britten aftes. 


\title{
19ineter Alst.
}

\author{
Sechs รahre ipäter.
}

Sehr ärmlich eingerichtetes Bimmer. Das einzig auffallende iłt ein

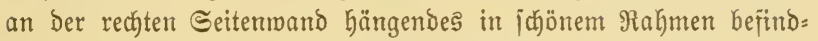

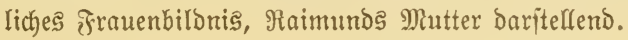

\section{Erite @zene.}

Raimuno.

Maimunt (bedeutend älter, in einfacher, aber fefr rein ges haltener Rleioung, tritt burch bie rüctwärtige Türe ein und feinen Şut und Stod ablegend jagt er traurig): Siicht mehr zu be= fommen, alle 5ुofinung vergebens! Die Füñe habe idh mir abgelaufen, aber es war ichon zu ipät! Beriest, verfallen und verfauft! SSerabe jebst, no es anfing befier zu werden, gerabe jebst, wo id) nicht mebr bem nacften Elend in bie Âtugen iehe. Das erite (Seld, das ich zulammenbrachte, benübte idh, um ifı mir noch zu retten. Jch glaubte, id) befäme ifn noch, aber es mar umionit! 2rlles umionjt! (Sieft auj ben Eleinen Jinger

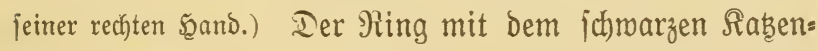
auge! Das lekte $\mathfrak{A n d e n f e n ~ a n ~ m e i n e ~ M i u t t e r , ~ b a s ~ f i e ~}$ 
jelbit getragen und mir geichenft hat, als ich nod) ein junger Buriche war. Das lebste 2 Indenten an fie, an meine arme, liebe Mutter. (B̈eft zu dem Bilde an ber $\mathfrak{W a n d}$

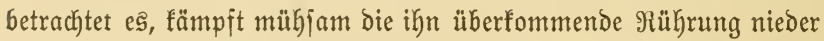
und flagt). Miutter! Meine SMutter! Sei nicht bös, ich fann nichts bafür. Ş(f) habe getan, was ich tun

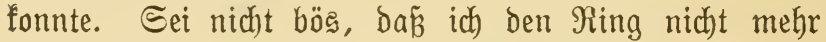
hab, ich) hab ifn immer in Ehren gehalten. (Rü̈pt bas Bild.) Schaut mich nicht fo bös an, Mutter. S(h) war ja nicht leichtfinnig; aus Rot hab ich ihn veriebt, aus großerer, harter Not, Mutter! S(ber es ijt mir fo idblecht

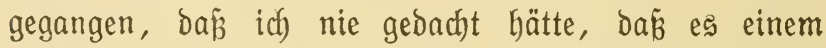
Menichen fo idhlecht gehen fönnte. Ess war mein lekstes Stüct, das ich meggeben muß̈te. Şabe jonit nichts mehr gehabt, Mutter. Şch habe feinen ganzen Stiefel mehr bejeffen, mein $\mathfrak{T}$ nzug mar gefficft und abgetragen,

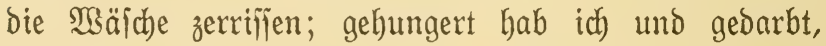
aber beinen Ming, Mutter, beinen Ming habe ich nicht meggegeben. Micht um eine Melt! Dann aber, Mutter, (itocft) ... Dann aber ijt's io ichlecht geworden, oh! fo jollecht, (ichüttelt fich ichaudernd) ba hab aushalten fönnen und hab ben ring veriebt.

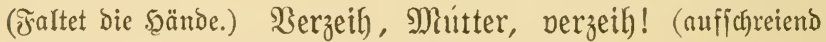

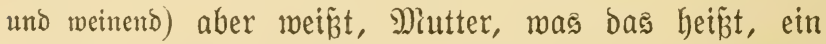
paar Tage nichts im Magen haben uno auf ber Strape herumirren und nicht wifien, wo am खlbend jein mübes Scaupt hinlegen. Mutter, mei liebe, gute Miutter!

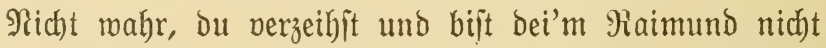
mehr bös, denn id, bin Dabei ein orbentlicher Mienfich geblieben und idh taan bir offen und ehrlich in bie 
Alugen idau'n, Mutter, wenn es aud) nur bein Billo

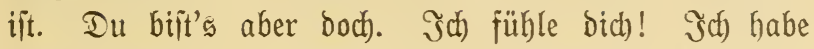
bich)! (2uffichreiendo.) Funger, Fiot, Frantheit, Fummer, Sorgen, Siedertracht und Ssemeintheit habe ich fennen lernen müijen, feine Mienichenieele, die mit mir gefühlt ober mir geholfen - (itol $l_{z}$ ) ich habe auch niemanden barum angejprochen - meder ben reichen $\mathfrak{B a t e r , ~ n o c h ~}$ meine (Sejchwijter, welche mich mitiammen verfauft und um die (Erbichaft, melche bu, gute liebe Mutter, mir zugedacht hait, betrogen haben. Tiemanden habe id gebabt, niemanden auf Sottes weiter Melt, ber mit mir gefühlt und gelitten, war nur immer allein auf mich jelbjt angeniejen. Siemanden sabe ich gebabt, nur bich, Mutter, und ben Şerrgott! Mutter! テ̌ch habe es gemubt, wenn es noch jo arg fäme, Jghr wïrbet mich nicht verlaifen und ich habe mich nicht getäuicht. Jd) bin nidjt untergegangen. Schwer ift's geweien, jehr idwer! die Whucht der Lajt hat mich nahezu er: brülft, noch iift es ichmer, aber bas idnwierigite hab ich, bir, Mutter, fei's Ianf', hoffentlich hinter mir. İh jehe nicht mebr ber nacften Rot und Dem fürchterlichen

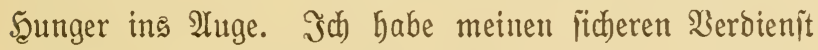
und mit bem eriten (Sielde mollte id) mir ben ßing mieber fichern. Es war aber zu ipät! (’3licft bittend auf bas ßild.) Nidft wahr, Mutter, du bift nidjt mehr bös, ou ichaujt idjon mieder lieber orein. (Frenbiger uno Goffnungsvoll iprectendo.) Bielleicht lann ich) ben fiäufer auftreiben und wann ich meinen ganzen Monatsverdienjt

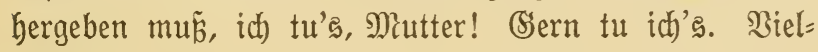
leicht befomm ich ben Ring boch mieder. Nicht mahr, 


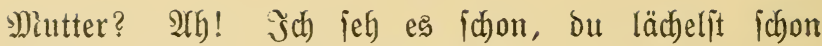
wieder. Iu bijt nicht mehr bös, bu bijt mieder gut auf Dein'n Bub'n, Mutterl! Mutterl! (WBif bas Bird

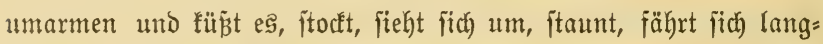
jam nit der 5̧and über bie atugen und fagt wie erwachend in

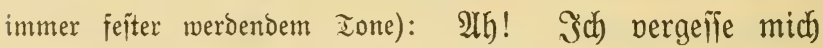
immer wieder und lafije mich Ginreipen. Es ijt mir, als ob idc) bie Mutter noch oor mir hätte und nicht ifhr Bild. Es ifit mir oft, als wäre fie nicht gejtorben, als bätte idh fie noch neben mir. Эch fühle ifre geiftige

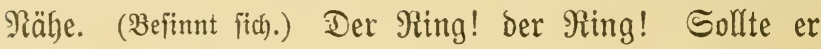

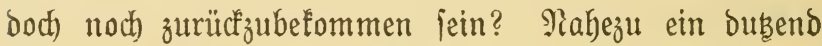
Sabre habe idf ifn gebabt. Smmer noch habe idh mich dagegen verwahren fönnen, deşelben verluitig zu geben, aber biejes Mal war's vergeblich. Und gerabe jest, wo es mir nach fo langer, harter Beit gelungen iit, ein fïcheres \$läbchen zu finden, aus welchem mich all ber

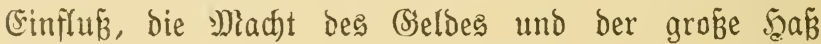
meines Baters nidjt mebr wertreiben fann. Es mar eine bittere, eine harte Beit. Marum er'mich wohl fo verfolgt? Warum?! Meil id ben Mut der Mahrbeit habe. Meil ich es ifm ungeidseut ins (sefictst ge= ichleudert, baj er mich nicht nur um meine mütterliche Erbichaft, fondern aud) um ben 2 (1tsgleich betrogen habe. SBeil ich jeine Sionfubine Dirne geheipen uno Mutter, Mutter! (Fieht auf bas 3irb) meil ich immer an bir ge= hängt bin. SBarum foll ich nicht an bir hängen, Miutter, mannit auch ichon lange tot bift, ou einzige, treute, gute Menichenieele, die id fennen gelernt habe. Iu hajt mich als Sind behïtet und als bu geitorben und idh 
als blutjunger Buriche rücfichtslos in bie Melt ge= itopien wurbe, hat mich immer ber (Sebante an bich aufrecht erbalten und mein (S)laube an (S)ott, den (S)ott, ben ich in meinem Innnern trage, nicht ben, welchen bie Menichen äuñerlich zur Schaul tragen. Was habe idi) Denn in ben langen Jahren nicht alles mitmachen miilfen?! Was nidht alles?! Unbarmherzig hinaus= geworfen! meine Stubien aufgeben milifen! ben Rampf nicht nur ums tägliche Brot, nein! jonbern auch gegen meine allernäd,jiten Bluţwerwandten, gegen beren SSemein= heit, Siedertracht, מ̧interlijt und Berleumbung. IAnd

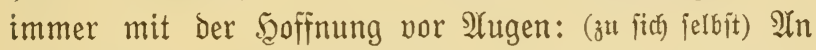
dem Iage deiner (Sroß̋jäbrigfeit wirit du wenigitens ein fleines Sapital erlangen? (Eächelt jarfajtija). WBirit bu erlangen?! Sollit bu erlangen?! Sa! Soljit bu, aber ich habe es noch nicht, denn als ich gropiällyrig

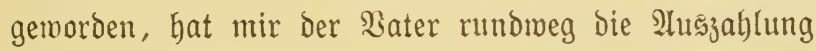
verweigert mit ber Begrïnoung, ich habe nichts zu be= fommen. Sch habe mich baher bemüft, idh habe getan, was in meiner Macht gelegen war, um mein Erbe zu erlangen, aber am Ende lief alles immer wieder auf Den einzigen $\mathfrak{3 e g}$ hinaus: Rlage gegen meinen $\mathfrak{B a t e r}$ ober (Iangiam) Strafanzeige eritatten, (abwehrent bie Şand ausitreffend) bas fann ich nicht tun, bas werbe ich nicht tun. Entieblicher Siedanfe! Ein Sohn gegen Den Bater! Da jei (Sott vor und wenn ich Darüber zugrunde ginge oder er mich zugrunbe richten mïrde, (energiich) aber bis jebst ift es ifm noch nicht gelungen uno jebst, burch ben jahrelangen Rampf und bie bitteren Erfahrungen bärter geworden, wird er mich nod) weniger niederringen. 
(Bejdyörend.) MSenn mich der Şerrgott nicht nieder= \{chlägt, den sienichen wiro's nicht gelingen! Jest meniger nod) wie ehemals! Sidht wahr, Mutter, (wendet

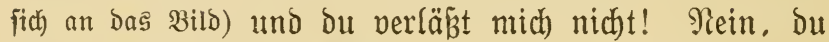
nicht! (Raimund hordft auf.)

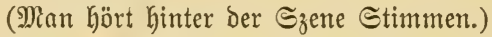

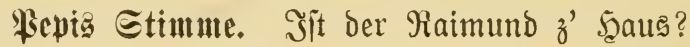

3immerfrau Stiumte. Mer? Der Maimund? Meinen's vielleicht meinen 3immerherrn, Den Serrn von Frebinger?

Bepiz Gtimur. Ra ja! Mem benn fonjt?

Bimmerirnus Stimme. Sa, ber ift grad o' Şaus fommen. Soll idh Sie anmelden?

Pepiz Stimute (ungedurdig). Was §hna net einfallt. Lafien $S^{\prime}$ mi nur eini, i bin ja fei Bruber.

3immerfraus Stimme. 2(y)! dann entiduldigen $\mathbb{S}^{\prime}$ ichon; das hab i net g'roukt. Da is dem Sherrn Frebinger feine $\mathfrak{I} u ̈ r !$

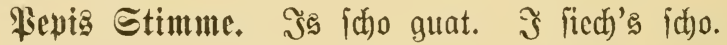

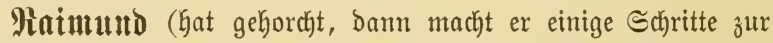
Türe und jagt lächelno): Mir icheint, Das ift ber \$epi, der mit meiner 3immerfrau itreit't. Muß gleich nachjeb'n.

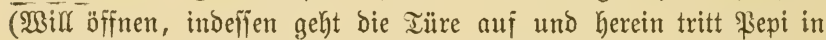

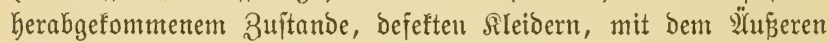
eines in $\mathfrak{A}$ nfangştadium befindrichen Trinfers.)

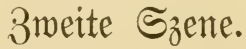

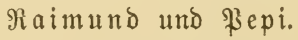

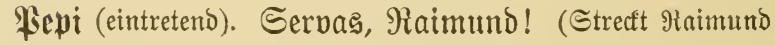
bie Şand entgegen, in welche biejer einjohlägt.) 
Raiunud. Servus, Repi! Mas fülyrt denn dich zul mir her?

Pepi. 马' reden lyab i mit bir uno no dazua prefijant.

Minimund. Dann ichiés nur los.

Pepi. (Slei mir i's angeh. Arber z'erít muaź i bi Do frag'n: Seit mann machjt benn bu folche Sponponaden, dá̉ ma bei bir net amal mehr eini fann?

Maimund (lächelnd). AYh! du meinft wegen meiner Quartierfran?

Pevi. So an alte Sifit'n; hat z'erit moll'n, i foll ihr a lang's und a brat's berzähl'n, wer $i$ bin, bis $i$

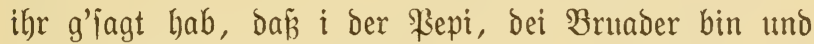
fie anfach auf 'o' Seit'u g'ichob'n hab uno eini bin.

Minimund (freundrich). Sie hat bich nicht gefannt, Bepi, und brum hat fie bich gefragt. Sie fann bodh nicht einen jesen fremben Menichen einlaijen.

Pepi. I hab mir eh benft, (jieft fich im Zimmer um) entweder bu bijt ganz veriduld't, da Ba d' nia z' Şaus bijt, mannit a z' Salus bijt, ober bu bift fo noblicht

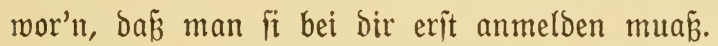

Maimund (belujtigt). Wseder bas eine nod) bas andere. Meine Schulden zahl ich nach und nach $a b$ und nobel ift's bei mir nicht, wie ou wohl jelbjt fiehjt, Denn bann wären wir beide noch Sav'liere.

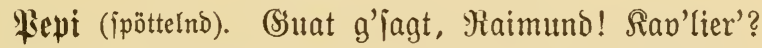
Şa! Şa! Şa! Itber fitier! Dös is vorüber. (Ernjter.) Bei jolddenen Berbältnififen, wo ma oft net von heunt

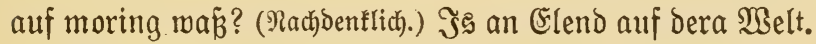

Rainuutd (ihn bedauerno und mitleibig anjehend, mit tröittender Stimnte). Ein (Şlücf auf jeden Fall nicht. AYber 
meil mir ichon babei find, \$epi, wie geht's bir benn immer? Mir hab'n uns ichon lang nicht g'ieh'n!

Pepi (zögernঠ). Na! Befier is net ror'n, eher

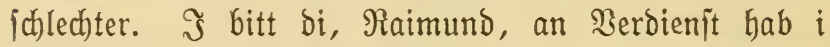
fan, wann $i$ mi a no jo anitreng, weil ber Illte ben Reuten immer porichimpft, i bin a Rump, a Irinfer und a Drahrer. S'eb'n tuat er mi a nir und bie Edhivejtern ... i bitt bi ...

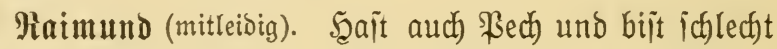
beinand?

Fepi (achielzutéeno, refigniert). Na, guat net. 3’ meng

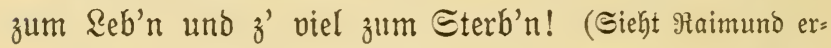
martungsvoll an.) Mir icheint aber, Raimund, bu bijt jeşt beifer oran.

Maimunt. Danfe, Repi! Es geht. (Bögerno.) 2tber, Hepi, ich bin nicht reich) ... meiśt (ipricht ver= legen) ... mannit aber möd)tejt. . fönnt $i$ bir aus= Gelf'n mit ein Bifierl. . .

Hepi (ïeft ihn bantbar an). Wanuịt was abgeb'n

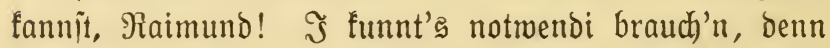
$i$ bin ganz ichroarz. Alber $i$ glaub, $i$ wir bir's balb z'ructzahl'n finna, denn bejtweg'n bin $i$ ja herfumma,

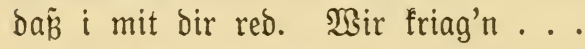

Maimund (unterbrectend). Mieviel braudjerit benn, SPepi?

Bepi (zögerno). Mannĩt ma balt bermeil a paar Sechjerln geberit.

Raimund (eritaunt). Ein paar Sechjerln?!

Pepi (ängitliç, zu viel verlangt' zu Gaben). Эït's dir z' viel? 
Maimund (mübjam feine Bejtürzung verbergent). 2(ber nein, Sepi, nein, bas iit mir nicht zu viel. (Steht auf.)

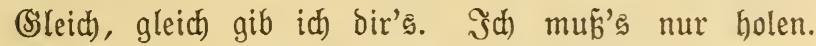

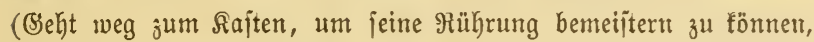
uno jagt wehmütig): (Sott! (Sott! Der arme Ieufel! Ein paar Eechjerln uno bie traut er fich nidjt mehr zu ver= langen. Nicht einmal pon mir! Mú bem aber ichlecht gehen! Da bin ja ich jebet vermögend bagegen. Mlutter, menn du das erlebt hättejt. 2lber viel anderes Bseld, als bas für ben Ring bejtimmt gemejene, habe ich jelbit nicht; ich werde ifm hievon geben. Die Mutter hat ifn auth fehr gern gehabt, (mirft einen Blif auf bas sirts) fie wird mir deshalb nicht böje jein. (Er hat fid , bies iprecheno, icheinbar am ßaiten zu idaffien gemacht, bie Börje aus feiner Iajche gezogen und fommt nun mit ber geöfifneten Börje zu

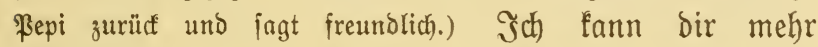
geben, Repi.

\section{Fiepi. Ias mär mir jefre recht.}

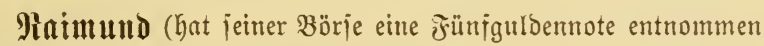
und gibt fie $\mathfrak{P}$ epi). Da hajt, Fepi.

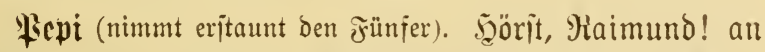
Fünfer! bifit benn ou Fapitalijt wor'n ober hajt an Scaupttreffer g'macht?

Raiunutd (Iächelno). Rein, Fepi, weder das eine, noch das andere, benn wenn dem io räre, ginge es uns beiden befier.

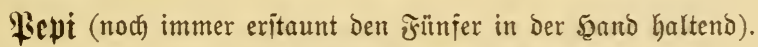
Daf́ d' aber glei an Fünfer hergeb'n faunit?!

Maimund (Tegt jeine Şand auj łepis હdjulter, zögerno uno 


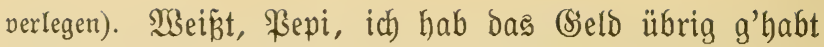
für . . .

Hepi (einfalfeno). $\mathfrak{J}$ jag's ja eh. Du bijt f̧ho Sapitaliit wor'n und legit beine (S'elder an.

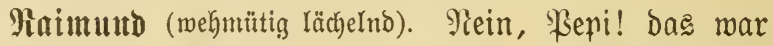
für einen ganls beitimmten 3 wect und nachbem ich biejen Zinedf nicht mehr erfüllen fonnte, fo . . (zögerno) fo . . . iit's am beiten, wenn ich's dir gib. (গn plöblich) auf= wallender (Semütsbewegung.) Pepi, du hait ja d’ Mutter auch gern gehabt?!

Pepi (vermundert). Db i's gern g'habt hab. Maann d' Muatta no lebert, war'n ma alle befier bran. Stber was willit benu jest auf amal mit ber Miuatta,

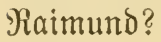

Mnimund (verlegen). Denť bir, Fepi, d' Mutter hätt' bir's gegeben.

Hepi (immer vermunderter). D' Muatta?! De is icho lang tot. Der Serrgott gib ifr bie emige Ruah!

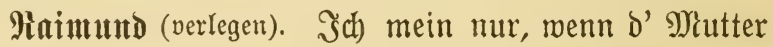
noch leben möchte, bätt fie bir's auch gegeben.

Hept. D' Muatta?! Uno daß̉ fie mir's geben hätt. De gebert mir mehr, als was hätt. N(ber lag ma, Raimuno, was manit benn eigentli mit bera Mieonerei von ber Mitatta? Mir icheint, oir geht $\delta$ ' Muatta no immer im Sopf rum und bu fannit es net vergeif'n?

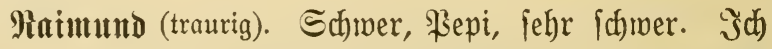
glaub, ich würbe nicht auf. fie vergefien und wenn id iteinalt mürbe. 


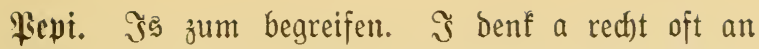
fie, benn es war all's anders wur'n, wann's no lebert. 2lber gar fo follit bir's do net in Sopf ies'n.

MatimultD (welcher traurig vor fich hingeblicft hat, ermannt

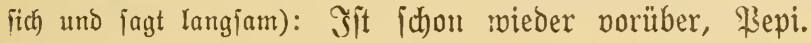
Die Mutter ijt tot und von bort fommt niemand wieder. Sit nicht mehr zu ändern. (Bejwingt fich, mit gefeétigterer

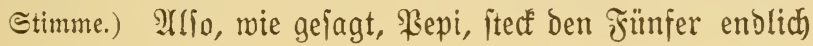
ein und...

Piepi (unterbrechend und dus Beld einjtectend). Danf́ bir

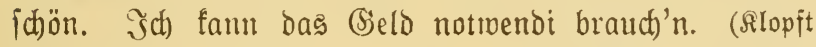
Raimund auf bie Schulter.) Bift a guater Rerl, roanmit a felber nix hajt, gibit do no was her.

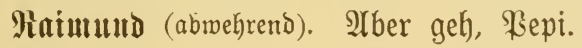

Fepi. Ret fo wia d' Schwejtern, be fo neibi jan, dáb an am liabit'n no was megnehmen möcht'n. (Tippt

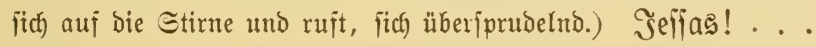
Jelią! Jebct hätt $i$ beim Disfurier'n beinah orauf

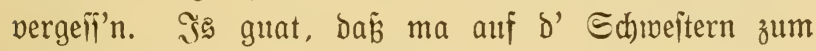
reo'n fumma fan. (Schlägt ficth auf bie Stime.) Ş̧ zu bumm und beftmeg'n hab i bi aufg'fuctst, (eifrig) bie (s'ichmitter hab'n ...

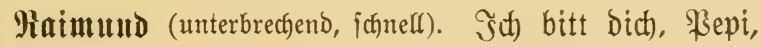
hör mir auf oder fang mir lieber nichts an oun ben (S)'idhwiftern, wo bie ihre Şand bintun, wachit mur $\mathfrak{H}_{\mathfrak{n}}=$ glücf für miereins heraus.

Pept. Saajt net Unrecht. 's is ja a (S'findo't. Utber desmal hafft's z'iammbalt'n uno brum hab'n's mi herg'idjicft.

SaIm, Fin ehridjes altes Săus. 
Maiumuto (vermundert). 31t mir haben fie bich ge= idicft? Die Sdyejtern?

Pepi. Dös hä́t die Sdyeitern und der Schubiaf.

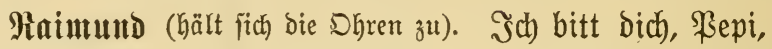
hör mir auf. Эch brauch nichts zu wififen, von bem Schubiaf, bem hinterliftigen Schuft, ichon gar nichts.

Pepi. Das itreit $i$ bir ja net $a$, daz ber Schubiaf a હ(f)uft is und bie Mitisi und bie Toni Menicher fan, aber desmal geht's net anderit, 's is meg'n dem 2 (tt'n!

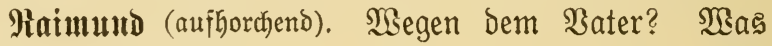
mollen fie mit ifm?

'Pept. Sosgeh'n moll'n i' auf eafm.

Raimund (gejpannt). Ŷuf den Bater? Sie find ja immer gut mit ifm!

Pipi (fopfichüttelns). (Suat?! (Suat?! Mia man's nimmt. Sann er eahna gnua gibt, lan's recht guat mit eahm, wann net...

Maimuti (einfalleno). গun und?

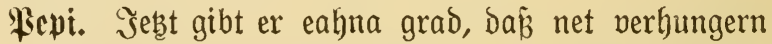

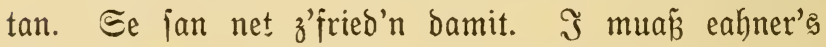
glaub'n, denn $i$ friag gar nix, bas fiechit ja eh).

Maimunt. Atber ber Schubiaf und feine Frau, bie Miki, haben boch Bermögen!

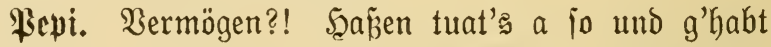
hab'n 's a amal, aber jebst lamentieren i' alleweil, ie hab'n nix mehr.

Maimunt. Das ift nicht mahr. Die haben immer geichmindelt und gelogen, bamit fie nichts hergeben brauchen und noch mehr bazu befommen. 
Hepi (zuitimmend). Das is a a io und i gab mir's a immer benft, aber hörit, Raimund, hajt denn bu icho

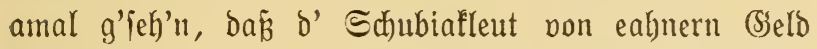
leberten? Ie hab'n do no allweil von andern Menich'n eahnern Seld g'lebt.

Raimund. Yllio und marum daun ...

Pepi (idnell einfallend). Ilno bie Toni hat gar nix, de lebt bon an Tag auf'n andern mitjammt ifgr'n Rino;

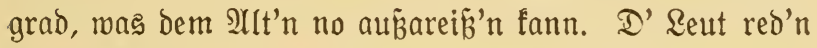
Denen Mab!'n eh icho alles mögliche nach), mur nix guat's. Dabet leb'n aber alle be Reut in bem $2\left(t^{\prime} n\right.$ jein (S)'ichäft mia o' jungen şund.

Raimund. Das habe idh ichon lange bemerft, da die Reute in Baters Seichäft eine gemififenloie, ver= lotterte $\mathfrak{B a n d e}$ find.

Pepi (erregt). II Bagaiche, de nur auf's Maub'n und Erpreif'n ausgeht und unieraner hat net amal zum leb'n. Und da foll ma zuichau'n (immer erregter uno eifriger meroend) wie all bas (S'findo'l lebt und g'niaß̈t und unieraner hat nir, z' eil'n. Şait benn du mas, Raimuno?! Du hait a nix, hajt nia nix g'babt. Şaft benn bu net a be ganz'n Jahr lang nix tan wia g'hungert? Dẩ bi jebst a bififerl derfangt hait, bas is eh a Munder!

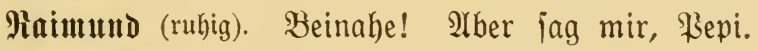
Mas wollt ihr eigentlich mit bem $3 a t e r$ tun, um bies zu ändern?

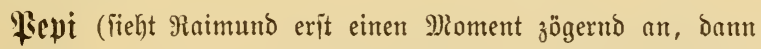
entichlofien, mit erhähter ভtimme). $\mathfrak{B a s}$ mer tuan wer'n mit'm $\mathfrak{A} l t$ 'n? $\mathfrak{i} b i b r a f \mathfrak{n}$ mer'n mer'n! 
Miaimund (eritaunt). AYbiorabn?!

Pispi. Entweder der Irte muß́ nachgeb'n oder mir wer'n eahm ben Şerrn zeig'n!

Maimund. Mas joll er nachgeben?

Pepi. Er muź uns geb'n, was mir brauch'n.

Maimund (bejtinmt). Эch nehme aber nichts.

Pepi. SMarum deun net? Scait es net notmendi?

Maimutud. Ias fchon, (erniten Iones) aber menn er mir's nicht freimillig gibt, erzmingen tu ich's mir nicht. Shr fönnt es meinetwegen tun, benn Not bricht (Eijen, aber wie wollt ifr das zuitande bringen? Mollt ifr ben Water flagen?

\{epi (ïberlegen lächelnd). YUber gar fa (S'jpur! SMir hab'n's alle latt bis bader (greift fich an ben şals mit

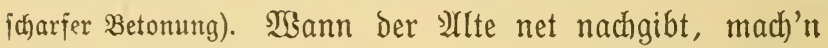
mer ঠ' Strafanzeig'.

Manimund (entieşt auffahrend). Strafanzeig'?! Den Bater?! Fiein, ba tu ich nicht mit, abjolut nicht.

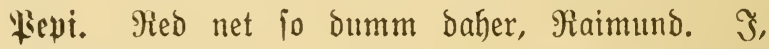
dei Bruader, i hab bir's immer guat g'mant. (Эn bes fehlendem Tone.) Mach lane Tanz, ou haltit mit!

Raimund (heitig abmehrend). Nein, ßepi, nein! Bei einer $\mathfrak{A}$ nzeige $(\mathrm{d}$ )on gar nid)t. $3 \mathrm{u}$ mas benn? SBenn ber Water fieht, dá̉ ihr es ernit meint, fo wird er eiuch) fo aud) geben, aber Strafanzeige?! Nein, nein, \$epi, das ijt zu viel, bas ijt zu meit gegangen.

Pepi. Larifari. Er joll ben Sirempel iteh'n lafij'n! Rnimund. Melchen Frempel?

Picpi. গia, 's (Sejc)äft.

Maimund (erjtaunt). $\mathfrak{A}$ h! um das handelt es fich! 
Siepi (unmutig). $\mathfrak{H m}$ alles mitanander. Er is iđjo z' alt. Reamo von uns is im (s'ichäft und ...

Maimund (unterbrechend, ironifaj). Ind der Schubial möcht bas (Seichäft in bie Sand friegen. Net?

Hepi (verlegen). Na ja, er will eini, aber wir a.

Maimund (einfallend). Эsh bab ja fofort gemuít,

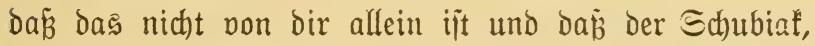
ber Sallunf, babinter itecft. SYlio wie gejagt: Solange ifr den Bater zwingen wollt, eud) ben Rebensunterbalt zu geben, gut, habe id) nichts bagegen, aber bei einer 2lnzeige .

Pepi (idguell einjaflend). Şaltjit net mit?

Minimund (enticjieben). Nein!

Fepi. Marum net?

Maimund. Meil's Der Bater ijt.

'Pepi (ironifa)). SEetl's ber Bater is! 's is zum Rachen! Raimund, bu bijt no alleweil der alte ein= bildnerifdye Rerl. Şat benn der Irte bir a nur amal g'holf'n, hat er bir net immer was antan, wo und wia er nur finna hat?

Maimund (rubig). Ess ijt wahr, was bu iagit, \$sept. Sch habe nie etwas gutes von ifm gejehen und bin überzeugt, dañ ich nie etmas gutes pon ifm zu erwarten habe, aber...

Hept. 2uber?!

Maimund (enticjieden). ES ift ber Bater!

Pept. Pa io was, Raimuno! Das geht idjo ins bouteillengrüne. (Stellt jich vor Rainumb mit in bie Seiten

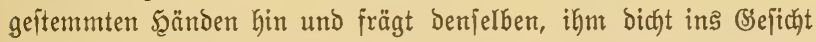


blifend): Maimuno! Bua! Maßst Denn bu, was dir Der 2 It te tät, เwann er funnt?

Mlaimund (rubig). So ziemlid).

Hepi. Umbring'n tät er bi, mann er funnt uno er net wuß̈t, baß ber (Salgen binter eahm jteht.

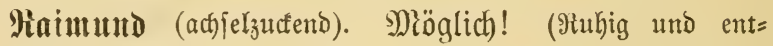
f(r)ieden). Hiepi, Rennjt bu bas vierte (Siebot?

Hepi (verblüfft). Das vierte (Sebot?! Das vom Battern uno Der Siuatta?

Maimund. Sa! (Mit erniter Betonung.) Efhre Bater und Mintter, auf baj es bir mohlergehe auf Eroen.

P'epi (vergnügt und frozzelnd). S(h)! Jekt maf́ i's, アaimuno, brum geht's bir gar fo guat, nur wirit bu babei immer dinner.

Matmund. $\mathfrak{H b e r}$ ich yabe mir nichte porzumerfen. Pept. Dafür der arte dir mehr.

Mininund. Du lieber Simmel! Etroas anderes habe idh von ifgm nicht ermartet und (sierechtigfeit ichon gar leine.

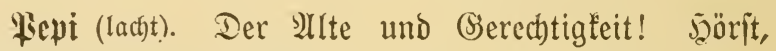
Maimund, ha, ha, ha!

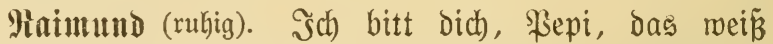

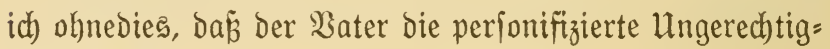
feit ift, aber...

Fepi (ungeduldig einfalleno). 2llitern, bann mach fane

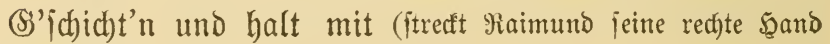
entgegent).

Maimund (entidjieben). Slein!

Fiepi (erregter). Du willjt uns all's perberb'n? 


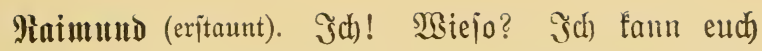
ja gar nichts verberben. Ish mache einfach nid)t mit.

Pepi. Alber das is net fo ohne, denn mannit net mithaltit, hab'n mer une umajunit ang'îtrengt.

Raimuul (eritaunt). Marum benn? SBir reden boch erjt Darüber.

Pepi. Alh na! M্Sir hab'n icho ...

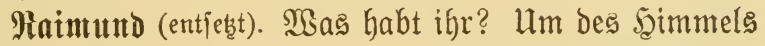
Millen, ihr habt boch nicht bie Strafanzeige gemacht?!

Bepi. গo net. Slber wann ber Irlte jebt net nach= geb'n hat, jo twirb's gemacht. Se fan fichon zum Illt'n gang'n!

Minimund. Sie find ichon zum Bater gegangen! Wher alles?

Pepi. Die Mithi, die Toni und ber Schubiat.

Raiumund. Der \&ump múp überall dabei fein. Und was mollen fie beim Bater?

Hepi. Er joll bie ganze (5rbichaft auszabl'n uno's (S)'ichäft hergeb'n oder...

\section{Maimund (geipannt). DDer?}

Fept. Doer ie mach'n meg'n all'm, was vom Erb= ichaftsprozéz wifiן'n, bie Strafanzeig'.

Miniutund (entjeţt). Die Strafanzeige gegen ben Bater! Sicht nur bie Erbjchaft foll er hergeben, fondern

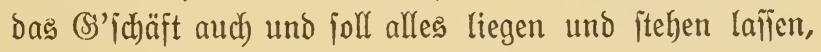
fonit (icaudernd) verfällt er bem Sriminal. Du, ßsepi, bas ift nicht nur eine gemeine Erpreffung, fondern auch eine große \&umperei und zu fo was bin id nicht zu haben. 
Pepi. צh was! Red net fo g'idhwoll'n baher. Fïr an \&ump'n g'hört a sumperei und der Allte is nix mia an alter \&ump! Da braucht ma fi fa (S'wifi'n braus z' mach'n.

Raimutnd (entietst). YHber Repi! Du marit ja nie ein ichlechter Menich. Bedenf, es ift unjer Bater!

Pept (zornig). Batta! Batta! Du bijt a Irott'l,

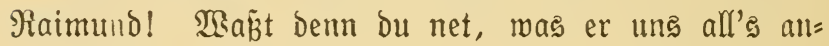
tan hat?

Miaimutud. So ziemlid).

Bept (in heller 2 tufregung, bemüht fitch, ę SRaimuno begreif=

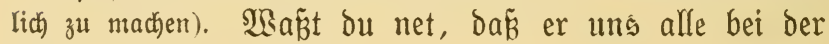
Erbichaft betrog'n hat?

Maimund (rubig, in bejtimmtem Ione). Das meí̄ ich!

Pcpi. Ş马 das fa Rumperei vom $\mathfrak{A}$ 't'n?

Raimund. Stimmt! Es ijt cine Rumperei, aber... aber ...

Hepi (halb verzmeifelt). Raimund! Raimund! Dut bifit ja funit io a g'idjeiter Rerl. Suat jei is idjön, aber bas nenn' id icho oumm iei, blunzenoumm. Mir hab'n do no a wengerl was friagt, du aber gar nix, bi hat er um all's bracht.

Maimuto. Du hait vollfommen recht, Repi! Er hat gelumpt und betrogen uno hat an mir erbärmlich gehandelt, aber er ift Der $\mathfrak{B a t e r !}$

Pepi (zornig). Immer lagit mer, er ift ber Watta! I laub'rer ßatta!! Şat er je g'fragt, ob wir bie Rinder jan, wia er uns um b' Erbichaft betrog'n hat und no bazu mit was fïr 'Mittel er babei g'arbeit hat. Man münt rein glaub'n, ou wußstejt's nöt? 
Minimunto. Das meins ich fchon lange.

Fepi. Das fagit aber io itab, dañ d' net all's wifi'n fannit, den juniten münteit ganz anders red'n.

Raimutud. $\Im$ (d) glaube nicht.

Pepi (zornig). Die \$Beterlin hat falich ausg'iagt und bie Cilli hat falid) g'idhror'n!

Maimutญ. SBeíz ic)!

Pepi. Uno ber Recfer, ber ausg'frefi'ne Mad'niact, bat falich ausg'iagt und an falich'n (Eid a'g'legt!

Minimund. Iavon hab idh gefört. Bon bem (see findel ift nichts anderes zu erwarten, die find für (S)eld und Borteile zu allem zu haben.

Fepe (Gițig). Hno Dofumenter hat er a g'fälicht!

Miaimuı (eritaunt uno erregt). Dofumente! gefälicht! bavon weiß ich nidsts!

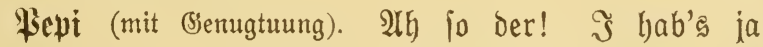

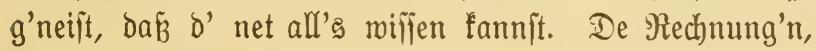
be auf d' Muatta g'idjrieb'u mar'n, hat ber 2ulte burch'n Recfer auf fein Ram umidreib'n laff'n und

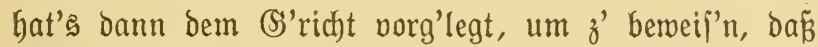
das şaus allweil eahm g'hört hat.

Minimund (empört). So eine Semeinbeit! Eine Niederträchtigleit! Ein elender Sdfuft, diejer Recfer!

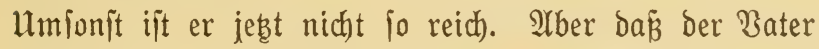
fo was getan hat? Es ijt nicht zum glauben!

Pepi (bähnend). Du haltit den $2\left(t t^{\prime} n\right.$ no immer für an Ehr'nmann und dermeil is er a ...

Raiumu (einfalleno). Ein Berbrecher, ein Schuft,

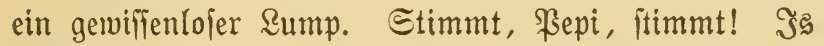
alles richtig. (Die Şände ringend.) 2Yber um bes Şimmels 
SBillen, er ijt unjer Bater, Pepi, der Mann von unjer

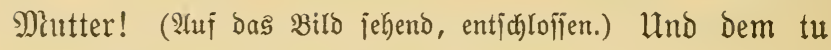
ich nichts zuleide, jei es, mie es jei!

Hepi. Uno gehit dabei z'grumb?

Maimund. Da ijt noch lange hin uno felbît wenn dem fo märe, io gebe ich als Menich zugrunde, als Menich, ber vor feinem eigenen (Seiviffen rein baiteht. Sieber als Menid) iterben, denn als Sump im über= fluije leben.

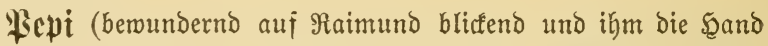
auf bie S(f̧ulter legend). Raimuno! Bua! Mann ma má̄, was bir ber Illte antan hat und was er bir antun möcht, wann er funnt; wann ma waś, was b' mit= mad'n muafist und wia bu bi burchg'hungert hajt (mit erhobener Etimme) und wann ma bi auf all bas 'nauf no fo guat, fo liab reo'n hört, (nimmt raimunos Ropi zärzlid zwifdjen jeine Şände, Galb meinerlichen Iones fortfahrend) und wann una waßj, mas für a rarer ordentlicber Mienich

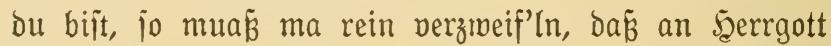
und a (Serechtigleit gibt, oder ma muá̃ î benf'n, bu bijt net ganz beinand, Raimund.

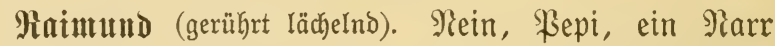
bin idf, Ssott jei Danf, nod) nicht! Man fönnte es

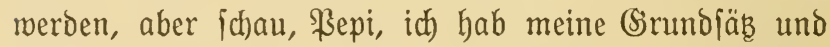
Frinzipien, von de geh ich nicht $a b$, ob gut, ob fablecht, burcb bick uno büun, uno menn's auth pon allen Seiten auf mich einmettert, ich) la $\tilde{\beta}$ nichl locfer, ich halte an denjelben fejt. Und wegen Der (Serechtigteit da oben, (Deutet Gimmelmärts) Du lieber Şimmel! Unjer Şerrgott hat fo viel zu tun, bá̉ er fich um den Einzelnen nur 
Don 3eit zu Beit umichau'n fann. Ienn ber Sïnder auf biejer Melt find mehr benn (Sierechte. Die gött= liche (Serechtigleit zögert oft lange, bebor fie richtet uno oft zu ipät, aber fie ridhtet immer gerecht. Du fennit ja bas Sprichmort: SSottes Mithlen mahlen langiam,

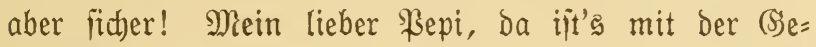
rechtigfeit auf Eroen viel ichlechter bejtellt, bie ijt io mie bie meijten Mienichen, haltlos! Die bequemt fich ebenfjalls den Berbältnifien an, neigt ïch nach der Seite hin, melche idberer miegt, (ironific) brum hat fie ja bie Mage in Der Szand.

Pepi. Tös is mir s' hoch, Repi. Das veriteh i nöt. Mer hat a Mag? Mas manit mit bera Mag? Raimund. Run, die Serechtigfeit, belier gejagt, bie bie SSeredhtigfeit poritellende (S)öttin Themis mit ber Binde vor Den IIugen, Der Mage in ber 5atio uno Dem Mithtidfwerte, Du wirit boch Deren Statue ober Itbbiloung ichon geiehen haben, Pepi?

Hepi. If ja! jeben fann i mi bran erinnern! ja! ja! jebst waj i's icho, das is be, be nix fiecht?

Maimunt. Das heipht fie fieht wohl und iit nidbt blind, aber fie hat eine $\mathfrak{B i n d e}$ vor den $\mathfrak{2}$ ungen.

H'epi. SBaia i's g'lagt hab, damit ץ' nix fiecht!

Raimuแd (ironifa). Bermutlich) zu dem Zmedfe, da=

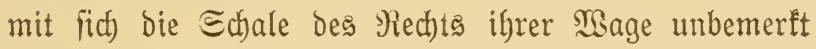
nad) ber Seite hin jenfen fann, auf weldher fich (Sield, Sunjt, Einflup und Macht befinden und bamit ïe es. nicht fieht, (mit beipendem Sarfasmus) menn ïe in $\mathfrak{A}_{\mathfrak{u}} \mathrm{s}=$

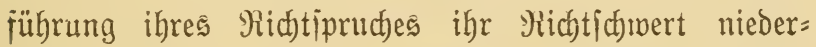
iausen läp̈t, basjelbe, of́t meben den હchuldigen nieder= 
faliend, den verbliifften Unichuldigen niederid)mettert. Sit's nicht io?

Repi (bewunderno). Maimuno! Bifi ou a harber

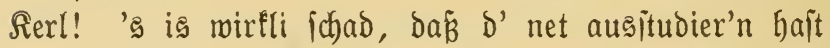
finna. Meiner Seel, Du rarit a guater Mrovofat mur'n!

Raimumb (abmefrend). Danfe, Repi! hab fein Ber=

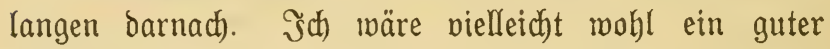

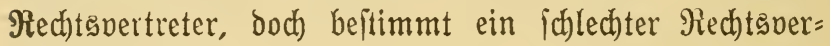
brefjer gemorden.

Maimund (Iachend). (S)uat g'reo't, Maimuno. Uno ou funntejt vielleidyt gar no Jujtizrat wer'n, wia ber Dr. Jeger!

Maimund (ipöttich). Ter hat farriere gemacht mit feiner Rechtsperbreherei; dem charafterlojen Rerl ijt's auf ein gebrodbenes (5brenmort oder eine \&umperei mebr ober meniger auch nicht angefommen, (jarfajitifa) weun es ifm nur was getragen hat. Arber was milljt benn,

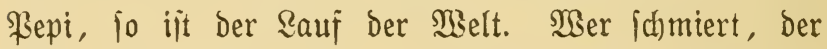

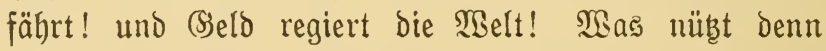

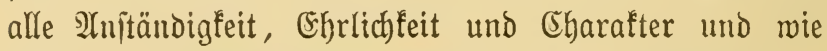
alle biefe ichönen Dinge heifen, aber jelten exiftieren, wenn das nötige (Seld hierfïr nicht vorbanden ijt. Illes! Illes! \$llunder! Siur für die faks!

Bepi. Und bei jolchenen ânfichten willit net mit= tuan mit uns?

Raimund (rubig und ernit). Bei folchen Âtnïhten?! Das find bie 2 (nficteten, die mir das unbarmberzige Reben und bie langiährigen, bitteren Erfahrungen auf= gezmungen haben, aber nur, um mir biejelben umio= mehr zur Marnung bienen zulaijen, fie nicht zu be= 
folgen und aus biciem (Srunde tue ich auch nicht mit geger ben $3 a t e r$.

Bepi. Hljo nicht?

Maimutto (entidjieden). Rein!

Pept. Ilber dagegen machit auch nichts?

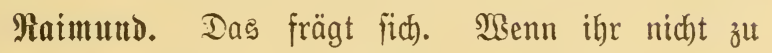
weit gebt...

Fepi (ichnell einfallend). Und wann's do weiter= gingert'n?

Maniunud (zögernd). Dann .. Dann (entichloffen) helf ich eher noch dem Bater!

Pept (erregt ausrufend). Paimund, bu twirit do net Dem $\mathfrak{A l t}$ 'n a no helf'n?

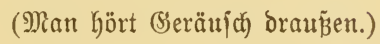

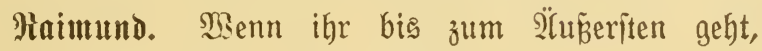
merde id ben $\mathfrak{B a t e r}$ bavor bebüten.

(Эn biefem Moment wiro bie mittlere Türe aufgeriffen uno herein itürzen Frresinger und (Eilli.)

\section{Dritte $\Xi_{z} e n e$.}

Die Sorigen, Fredinger und Cilli.

\section{Lepi (überraight). Der Alte!}

\section{Maimund (eritaunt). Der Bater!}

Fredinger (veritört, alt, gebrechlich, geht fo johnell es ify

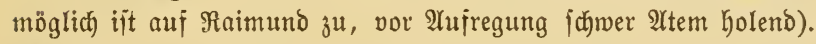
Sa! Dei Bater is! Raimuno! Dei Bater, der bei bir Şilf fuach)t. (Ïngittlich, weinerliden Tones.) Du tuajt net mit 
eahner! Iu wirit Dein alt'n Batern net ins Rriminal bring'n.

Maimund (fafilungslos). Arber Rater!

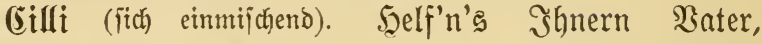
Raimund.

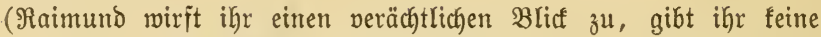
2nntwort und wenbet fith ab von ihr.)

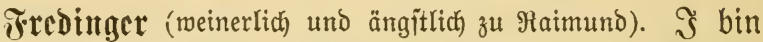
eahna dabong'rennt. Der Schubiaf und d' Mab'In mar'n bei mir und woll'n mi ziwing'n, $i$ joll all's lieg'n und fteh'n lafj'n und's eahna geb'n, funiten...

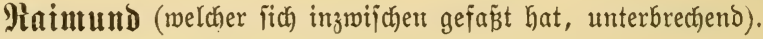

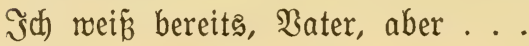

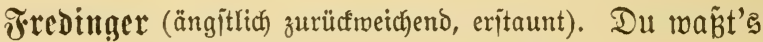
¡cho? (2Tngiterfült ausrufend.) Du wirît do net a auf mi losgeh'n, Raimuno?!

Raiumund (berufigend und tröitend). Du fannit ganz rubig iain, Bater, idh bin zu fo was nicht zu haben.

Fredinger (befreit aufatmend). $\mathfrak{I}$ hab icho glaubt ...

(Silli (einfallend). Das is ichön von Эhna, Maimund, helf'n's nur Şnan ßatern.

Raimund (ïh) brüsf zu Cilli mendento in erregt veractuntgs= vollem Ione, 2 ort für $\mathfrak{B o r t}$ jळarf betonend). Eriteng bin iđh für Sie nicht der Raimuno, jondern ein für allemal ber Jeer Fredinger. 3weitens iprechen Sie midh nie= mals wieder an, ba ich mit Berionen Jhres Schlages

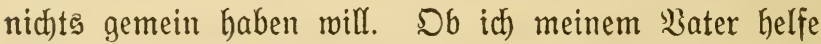
oder nicht, das iit meine Sache. (Scharf betomend, mit äzender כronie.) Sie fönnten es immer nur verderben. 
Üno nun (aนj bie Türe weifend) gehen Sie aus diejem 3immer hinaus!

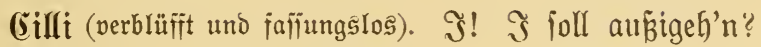

Fredinger (erregt). Raimuno!

Maimund (göflich, aber entichieben). Bater! Mas bich betrifft, das ijt ein anderer Fall, aber bieje.. Dieje

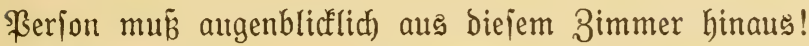

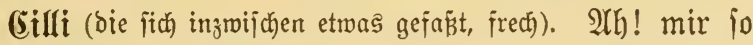
mas! $\mathfrak{A}$ io a Rectfheit.

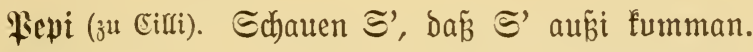

Miaimund (zornig). Sie freche \$Serion! Sinaus mit Shnen! Sie verpeiten mir bie Ruft. Das 3immer habe idh mit meinem idwer und ehrlich perbienten (Selde bezahlt, niuist io wie Sie. Ia ijt für Reute

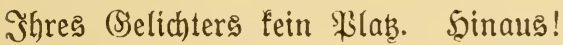

Frroituger. Raimunb! Sie lect mit mir!

Maimutto. Eben Desmegen.

(Cilli (bleich, veritört, babei freed). I bin an achtbare Frau!

Hepi (höhnifd lachend). So an alt's suader!

Manimund (zornig). Eine Pirne ohne Sittenbiichl find Sie. Bum lebsten Male: Şinaus!

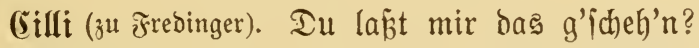

Fredinger. Raimund ...

Raiumud (idgnell unterbrechend). Bater, wenn bu ifr hilfit, bei Ssott! bann jollen fie mit bir machen was fie wollen. $\Im$ h) helfe bir nur um ber Miutter Millen und um ber Mutter $\mathfrak{B i l l e n}$ muß bieje Fierion hinaus. (Er weift nod) einmal nach ber Züre.) 


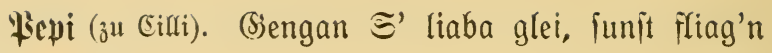
S' no außji!

Manimund (mild und zornig, totenbleich). Menn Sie nicht augenblicflich gehen. jo, bei (Sott! (frampft bie Şände zujammen) idh prügle Sie mit dem Stock hinaus. (Siegt (idc) fuchent im Bimmer um.)

Cilli (gegen bie Türe fannell zurüctweidhend, Iaut weinend und aufichreiend). Dh! Mein (Sott! Dh! Mein Sott! $\Im$ geh fido! ’a, io mas!

Pept (hat bie Türe aufgerifien). Schaun S', daḱ $a b=$ fahr'n, Se alt's Ruaber! Se jan am ganjen $U_{n}=$ glüh iduld.

(Cilli ab.)

\section{Bierte Szene.}

Die Borigen, ohne Cilli.

Minimund (Eilfi nad)rufend, empört). \&llder, altes, elendes! Mill mir mit ihrem Dirnenatem meine ehr $=$ liche \&uft verpeiten.

Fredinger (melcher hilflos zugejehen, ruft zornig und jitterno). Raimuno, bas is brutal!

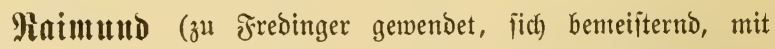
rufigerer und weidferer Stimme). Bater, perzeih! Sch fonnte nicht anders! Du fingegen bilt bei mir fo ficher wie in 2lbrabanis...

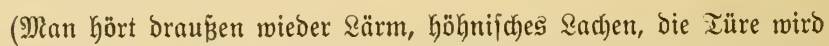
aufgeriffen und herein ftürzen lärmeno und polternd Schubiak, Miţi und Ioni.) 


\section{Jünfte Szene.}

Die Borigen, Schubiaf, Miţi und Ioni.

(Die leţten orei bedentend gealtert. Schubiaf bem 2lusjeben nach) verlebt und verfonmen, jalopp gefleibet. Miţi und Ioni verlebt, heruntergefommen und in jafreiende Farben gefleidet. Arfe orei hereinjtürzend mit höhnifhem lärmenden sadjen.)

\section{Mithi. Şa! Şa! Şa! De hat er g'liefert!}

Ioni. Uno mia! Mana fann de alte Mes'n a no!

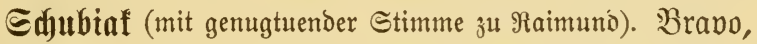
Raimunt, bravo! bie alte Bubälterin hajt ichon 'naus=

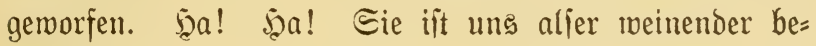
gegnet.

Mitji. Ssuat haift d' es g'macht!

Toni. Bijt halt do no immer ber alte Rerl, ber fane (s'ichicht'n madht!

Sdubiaf (aufmunterno). Da find wir ja ficher, bá̉ Du auch mit bem Illten nicht viel llmitände machen wirit?

Pepi. Die Cilli hat er auß̉ig'ichmilī'n, aber gegen ben $\mathfrak{A}\left(t^{\prime} n\right.$ will er net mittuan.

Mitbi uno Tont (verblüfft). Net? $\mathfrak{2}$ !h! das gibt's net! Sdubiaf. Der Raimund mús mitfalten, das werben wir erit jehen.

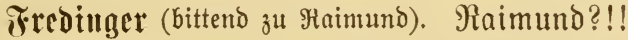

Maimund (meich). Sei nur rubig, Bater! Nichts Darf bir geicheh'n.

Sraubiaf (höhnija). Sgoher auf eiumal biefe Riebe?

Pinimund (zu Schubiaf). Das ift meine Sache und geht bich gar nichts an. Sch meís jelbit am beiten,

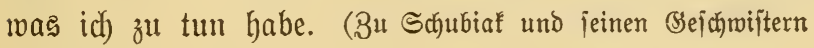


gemendet). Die (Eilli habe id hinausgejagt, weil fie eine elende, gemifijenloie ßerion ijt, bie an ber Mutter am meijten geïunbigt hat, aber gegen ben $\mathfrak{B a t e r}$ (mit erhobener Stimme) trete idh nicht auf. Jm (S)egenteil merbe...

Fredinger (banfbar). Raimund!

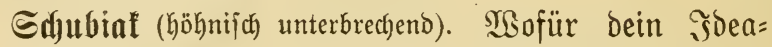

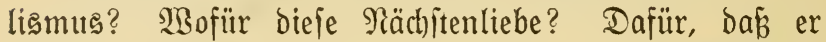
bich leit sahren gehajat, verfolgt und um bie Erbichaft betrogen hat.

Maimund. Das habe idh mit mir ielbit abzumachen. Unter feiner Bedingung gehe ich vor aegen Den Bater und (mit erhöhter, brohender Stimme) wenn ihr gegen ben Bater ernitlic, auftretet, fo merbe ich ifn ichiben wo und wie ich mur fann.

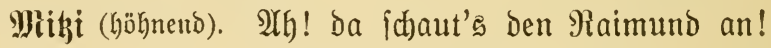

Ioni. 2llitern fo fchau'n ma aus!

Miłbi (höhnend, nit äł̧ender Schärfe). Das liebe, brave Bubi hat auf einmal fei Seer entrectit!

Srjubiaf (böhnif̧, beittimmt). Das werden bir erit

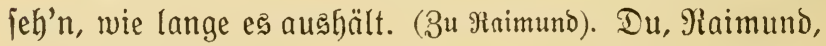
ber $\mathfrak{B} e p i$ hält auch mit, mur weil er alles meiß̄. Arber Du weißst noch nichts, orum merbe idh oid) ein menig

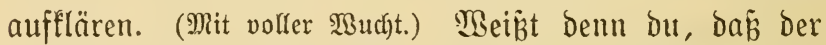

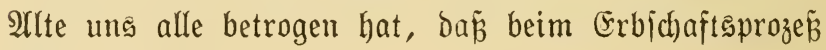
falich ausgeiagt...

Mithi (eimfallend). Ind falich g'ichwor'n wor'n is!

Toui (befräftigend). Sa! Maimuno, falid)e Eibe ian a'g'legt wor'n.

Pepi. Das wá̉ er eh alles! 
Mitji und Toni (eritaunt). $\Xi_{0}$ ! Ia milif'n mer no weiter berzähl'n.

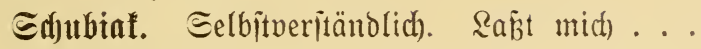

Fredinger (vortretend und unterbrechend, zu Rainumb und Ioni gewendet). (SIlaubt's eahna net alles. Ie hab'n euch felber um'n Dam braht. Te hab'n a jeo's non mir um iechstaujend (sulden mehr friegt wia ifr.

Raimund und Pepi (eritaunt und entrüitet). IYh! IYIg! Edultbiaf. Das ijt nicht wahr!

Bepi (zu §resinger). MBiajo Denn, Sater?

Mitji. $\mathfrak{A}$ jo a Rugenichippel!

Ioni. Der $\mathfrak{A}$ (te mill fïh) jeßst auşaliag'n!

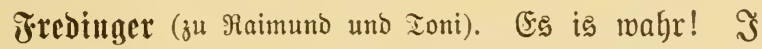
hab bie Beitätigung'n in meiner Eijernen, i fann's ja jeden Moment herzeig'n.

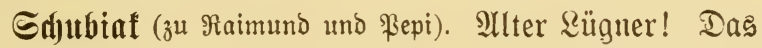
lagt er aber nicht bon ben g'fälichten Hechnungen!

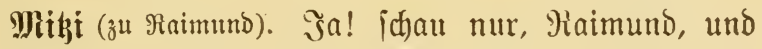

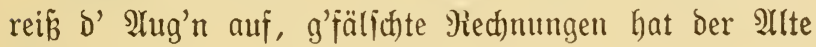
unterichob'n!

Jredinger (ï̈h aufrafiend, bas verzmeifelte Epiel zu ges minnen, zu Miţi, Toni und Sdyubiaf). Shr wollt's was von unterichiab'n reo'n! Shr, be ifre eurer Mituatta a falich Ieftament unterichob'n habt's.

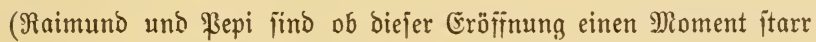
und faffungslos.)

Raimund (ermannt fitch und richtet an fresinger bie Frage): Mas, Bater! Das Teftament pon ber Mutter mar ge= fälicht?! 
(Toni, welche totenbleich) geworben wax, wirft mütende Blife auf Erebinger, ba z er fie verraten.)

(Schubiaf und Miţi find totenbleich) gemorden und finden in ifrer

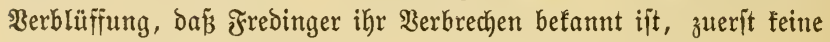
Worte, um ठann um fo mütender vorzubreçeen.)

Sdjubiaf (zu æredinger). Du alter Berleumber!

Mitji. $\mathfrak{A}$ fo a (S)emeinheit!

Toni. Niederträchtig!

Frediuger (zu Raimund und ßepi). Die Muatta hat euch) zwa foviel vermacht g'babt, wia den Mab'ln uno da hab'n's der Muatta bas faliche Teftament zum unterichreib'n geb'n.

Mitiji. Er liagt! Das is net mahr!

Sdubiaf. So ein alter Sauner!

Tont. SKer hätt fi denn fowas Denfen fönnen!

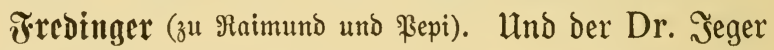
hat's aljer fertiger mitbradht g'habt, ber mar a mit im Bandr!

Mithi (ichreiend). Das is alles berlog'n!

Frrediuger. Mas derlog'n?! ('2uf Ioni meijeno.) Die Toni hat's mir ja felber berzählt.

(Schubiaf und Mif̨i wollen mütend auf Ioni lositürzen.)

Toui (verzmeifelt, gellend). Er liagt, ber ßater! Er liagt! `̧ bab nix g'iagt.

Frediuger. $\Im$ hab ihr orum no brei Iaujender braufgeb'n.

Tout (auffacreiend und geflend). Das is net wahr! (Bu Fresinger.) Du liagit, Bater! Du willit mi nur einibrah'n, weil bu bir nimmer anders helfen fannit.

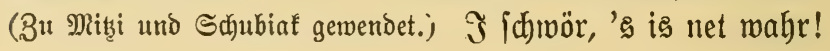


Sdjubiaf (zu Fresinger). (Şemeiner Rerl!

Mitji. So a Rieberträchtigfeit!

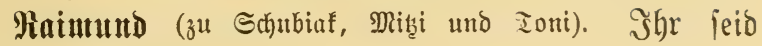
elende Menichen!

Bepi (zornig). Il mijerablig's Rlumpert feio's, a faliche Banda, uns io anz'ichmier'n. Da hilf $i$ ench a net. Der Raimund hat ganz recht, wann er lagt, er will mit euch nix z' tuan hab'n; es fummt immer nur an Unglïß und a (Semeinheit auß̃a!

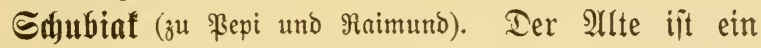
erbärmlicher \&ügner.

Mitji und Toni. Sa, fo is! Liag'n tuat er und (c)lecht mach'n tuat er an.

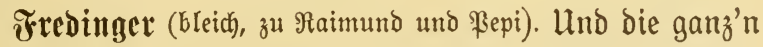
Jahr hab'n's mer (Şeld und wieder Bseld aupapreßßst und jeşt möchten's no alles. Shr habt's nix g'habt und de hab'n's verlumpt und veroraht.

Mitij. Das is net wahr!

Sdubiat. So ein alter Berleumoer!

Toni. Pix hab'n mer friegt!

Bepi (zonnig วu Sdhubiaf, Mithi und Toni). Şalt's bie (S)ofd'n!

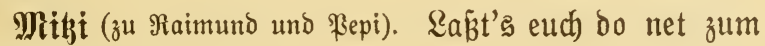
গarr'n balt'n.

Toni. SSlaubt's eabm nix. Seio's g'icheit. (Bese

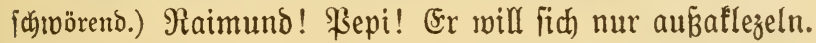

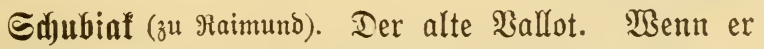
bätte tönnen, hätte er diđ zugrunde gerichtet.

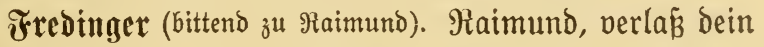
Batern net. $\mathfrak{u m}$ deiner Miutter Millen! 
Maimunt (mächtig bewegt). Rommt's wie's fommt, Bater! ร̧h helfe bir und Fepi aud).

Sdubiaf. Dummbeiten! Seio's doch nicht blöb!

Ioni. Mir hab'n ihm menigitens no was $a b=$ g'nommen, ifr aber...

Mihi (einfalleno). Ђ̉abt's gar nix friegt uno mollt's ihm no helf'n.

Fredinger (bittent). Siaimund! Repi!

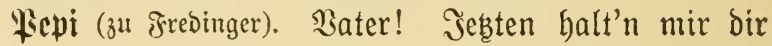
d' Stang'n.

Sdubiaf (mütend). Şhr ieio witflich fo bumm und idü̈b̨t Den alten (Sauner?

Pipi (ž Schubiaf). Şalt's Maul!

Maimund (zu S(jubiaf). Du bij̄t ein viel grö̈perer Scallunfe wie er und er ijt der $\mathfrak{B a t e r !}$

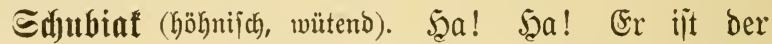

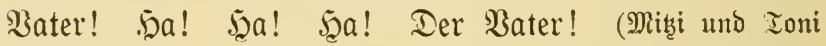
lachen ebenfalls höhnifd.) (Es ift doch zum lachen! (Mit

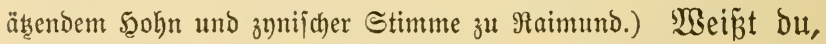
ou Soealijt, Du mieder auferitandener (Shrift, meißßt bu, mas ber alte Eyrenmann immer lagt, warum er bich immer jo gehaßst hat? SBeipt bu bas, bu braver Sohn?!

Mithi (aneifernd zu ভchubiaf). Mecht hait! Sag's ifhm mur, Damit er wein, wie er bran iit, bas unichuldige Engerl!

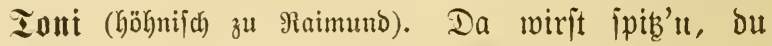
brav's Bubi!

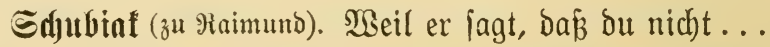

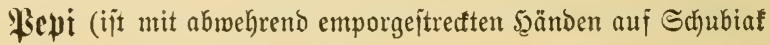

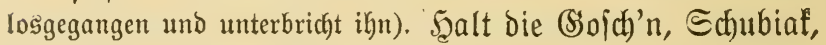
ou ridut'it an Unglitef an! 
Raimund (entichieden zu Sdjubiaf). Siur heraus bamit!

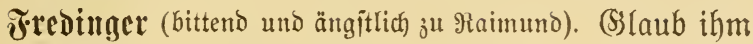
net, Raimuno, er liagt wieder!

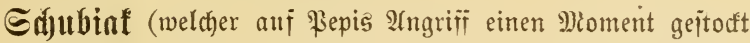

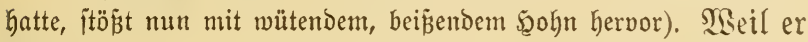
jagt, dấ bu nicht von ihm uno ein Saitaro bijt!

Hepi (zornig zu Sdfubiat, welder feig zurüufmeidft). Rausbua, bafür g'töreten bir a paar şatichen!

Maintund (iit juerít wie vom Blibe getroffen iteflen ges

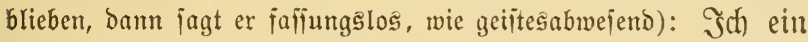
Baitard uno meine Miutter eine ichlechte Perion?! (Dann ermannt er fich) und jich an ben ängittich zuriidfweidsenden Fredinger mendend, zeigt er auf das Bild Der veritorbenen Frau Jresinger und frägt in Gartem, itählernem uno eifigem Ione.) Bater! Wei dem IIndenfen der gottieligen Mintter! Sajt Du bas gejagt?

Frredinger (totenbleich, mit Flehentlid) gefalteten bänden,

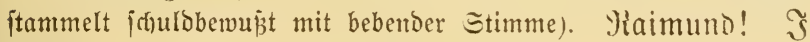
hab's ... net ... 10 ... g'mant.

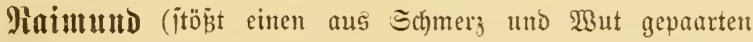

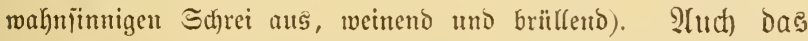
noch. Das iit ber Tanf für alles. Siicht mich ichändeit du, nicht midh, jonbern bas höchite, das heiligite, was ich beiefien, meine Mutter! (TSill auj sresinger lositürzen.)

Pepi (will Maimund zurüaffaltent). Yiaimund! BHa! Sei g'id)eit!

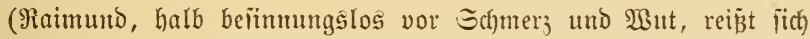
von \$epi los, pacte einen Stuhl, hebt denjelben nit beiden bänden

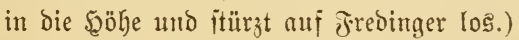

Frredintuger (mit flehentlich) gẹalteten 5ุänden, jărectensbleich, 
mie gebannt uno geiftesabivejend flüiterno). Raimuno, es iît...

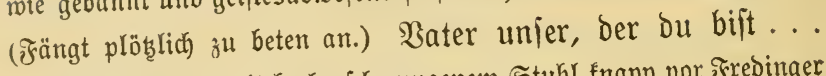

Raimunt (mit God)gefdrwungenem Stuhl fnapp vor Frebinger iteheno). .5uno! (Sottwermaledeiter, alter Şund!

(Mitic und Toni fabreien auf und halten fich bie $2(u g e n$ zu.)

(Fepi iter)t idyrecfensbleid) mit ausgeittrectem $\mathfrak{A r m e}$ ba, mit welchem er Raimun zurürthalten mollte.)

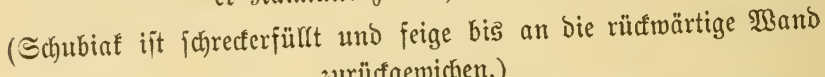
zurüdfgemichen.)

(Эn biefem Moment fintt Frebinger, vom Şerzichlage getroffen, entieelt zu $\mathfrak{B o d e n . )}$

Raimullo (iteft einen Moment wie itarr, Den Stubl hoch er= Goben, ba, bann falleubert er benfelben meit von fich, greift ficts mit Den Sänden verzmeifelt an ben Ropf und wirft fich hierauf fafiungs: log über bie Reiche Frrebingers mit Dem verzmeifelten, fidmerzourchs= bebten 2(ufichrei): Mein Bater! Mein armer Bater!

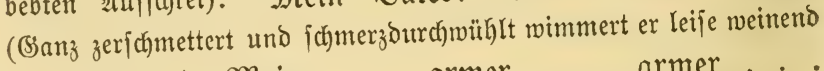
und ítón̈weife.) Mein . . armer . . armer . Bater!

(Borbang fällt.)

Ende bes vierten aftes.

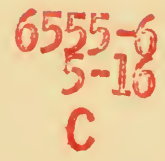




University of Connecticut

\section{Libraries}




\section{Bertha von Suttmer's Gesammelte Sdiriftell \\ Wohlfeile Lieferungsausgabe}

Vollstondig in ca, 60 Lieierungen d $40 \mathrm{Pf}$, $=48 \mathrm{~h}$. oder in ca. 12 Bänden brosch. d $2 \mathrm{~m}$. $=2.40 \mathrm{~K}$, geb. dे $3 \mathrm{~m}$. $=3.60 \mathrm{~K}$.

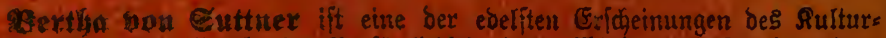

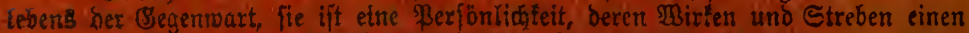

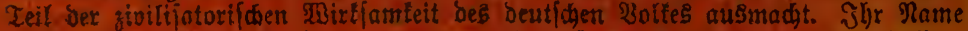

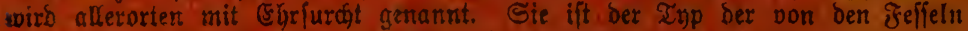

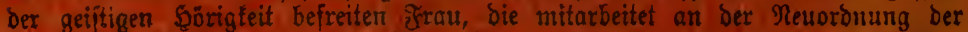

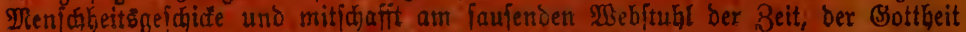

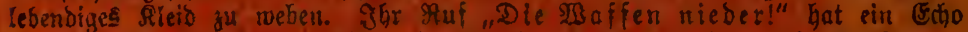
gefunben in alden sanben bis meit über bie Dzeane, er Gat grofe Ilaffen bes

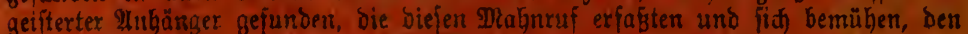

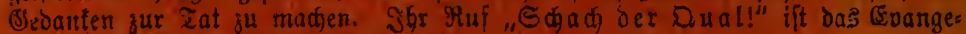
lium bet stroderner: Prenichleit genoroen.

Baronin Bertba non Suttner it aber nidht blos Die Reformerin, bie

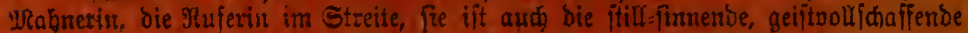

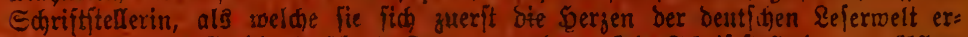

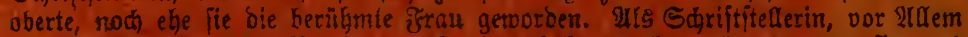

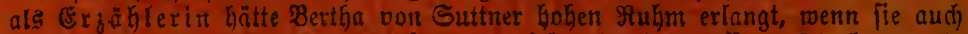
niemals bas Banner der Friedenşache mit feiter Sand ergriffen. Sie hat, nahs

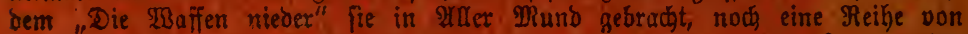

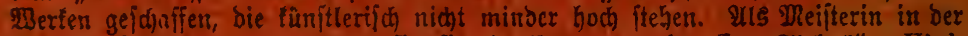

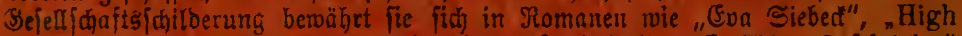
Life", "Tronte ot Quarante" uno anberen, fowie in ben "ErzăbIten Suitipielen"

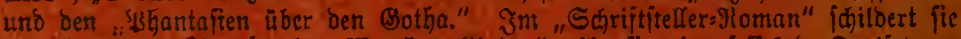

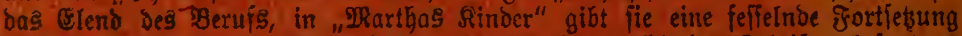

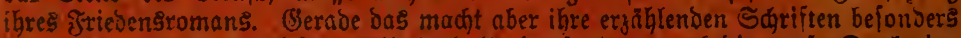
nertooll, baf fte barin nidit mur Unterhalterin, jonbern aud bic grofe Denterin,

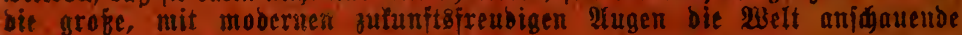
Berföntiogleit ift, bie fie uns fo lieb unb wert gemtadt bat. In biejem Sinne ift Bertha von Suttner gerabe in ihren erzäblenton Säriften eine Erzieheri"

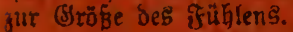

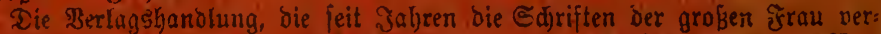

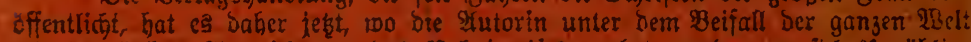

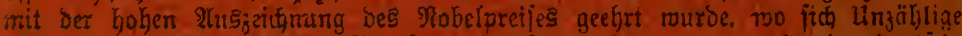

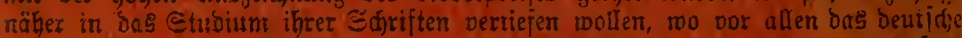
Molf in feiner breiten Maffe "bie Suttrer" aud) näher fennen zu lernen mủnjat.

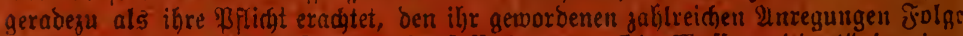
ju Feiften und bie EGriften ber Berfafferin von "Die Daffen nieber!" in einet

\section{billigen 2 olfaratsgabe}

al̆genein zugänglich ju maçen.

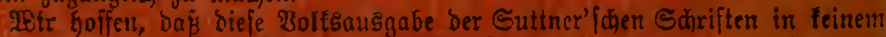

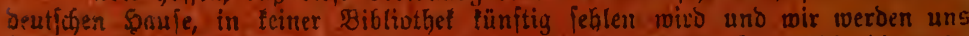
freven, roenn es uns baburk gelingt, ben hohen isoenlen, für melde bie melt:

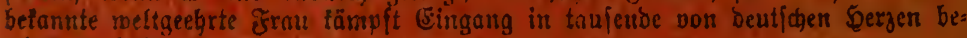
reitet ow habett.

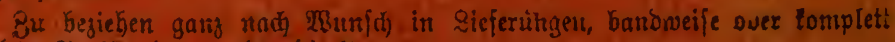
buch iebe Bucjtanbling ober birett pon

E. Pierson's Verlag, Dresden. 